\title{
Untersuchungen \\ am \\ Metacarpus von Lauf- und Schrittpferden, \\ besonders auf Biegefestigkeit.
}

\section{Inaugural-Dissertation}

der

hohen philosophischen Fakultät der Universität Jena

zur

Erlangung der Doktorwürde

vorgelegt von

\section{Albert Wolter}

aus Templin in der Mark.

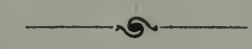

Mit 34 in den Text gedruckten Abbildungen.

Merseburg.

Druck von Friedrich Stollberg.

1907. 



\section{Untersuchungen}

\section{am \\ Metacarpus von Lauf- und Schrittpferden, \\ besonders auf Biegefestigkeit.}

\section{Inaugural-Dissertation}

der

hohen philosophischen Fakultät der Universität Jena

zur

Erlangung der Doktorwürde

vorgelegt von

Albert Wolter

aus Templin in der Mark.

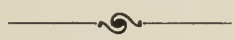

Mit 34 in den Text gedruckten Abbildungen.

Merseburg.

Druck von Friedrich Stollberg.

1907. 


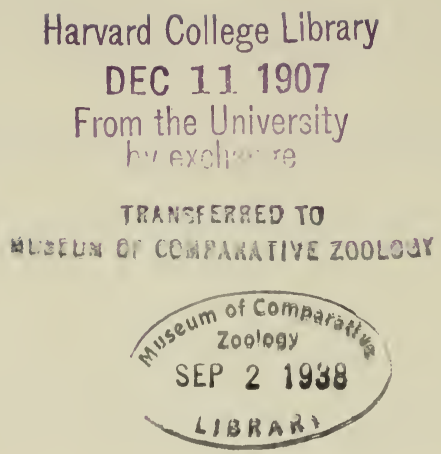

Genehmigt von der philosophischen Fakultät der Universität Jena auf Antrag des Herrn Professor Dr. S. von Nathusius.

Jena, den 26. Juli 1906.

Professor Dr. G. Goetz, derzeit Dekan. 


\section{Inhalt.}

Einführung . . . . . . . . . . 1

Das Pferdematerial . . . . . . . . . . . . . . . . . . . . . . . . . . . . 2

Das Messen der Pferde . . . . . . . . . . . . . . . . . . . . . . . . . . . . 4

Die Auswahl der Knochen . . . . . . . . . . . . . . . . . . . . . . . . . . . 6

Das Messen der Knochen . . . . . . . . . . . . . . . . . . . . . . . . . . . 6

1. Das absolute Gewicht . . . . . . . . . . . . . . . . . . . 7

2. Das Volumen . . . . . . . . . . . . . . . . . . . . . . . . . . . . 8

3. Das spezifische Gewicht . . . . . . . . . . . . . . . . . . . . . . 9

4. Die Länge der Röhren . . . . . . . . . . . . . . . . . . . . . . . 11

5. Der Querschnitt des Röhrbeins. . . . . . . . . . . . . . . . . . . . . 11

Die beiden Durchmesser . . . . . . . . . . . . . . . . . . . 12

Der mittlere Durchmesser . . . . . . . . . . . . . . . . . . . . . 13

Mittlerer Durchmesser im Verhältnis zu Röhrbeinlänge . . . . . . . . 13

Flächeninhalt des Querschnittes . . . . . . . . . . . . . . . 13

Grösse des Querschnittzylinders . . . . . . . . . . . . . . . . . . 14

Knochenstärke und Röhrbeinumfang . . . . . . . . . . . . . . . . . 14

Die typische Belastung der Röhrenknochen . . . . . . . . . . . . . . 17

Wandstärke . . . . . . . . . . . . . . . . . . 17

6. Die Griffelbeine . . . . . . . . . . . . . . . . . . . . . . . . . . . 24

7. Zusammenfassung der Messresultate an den Röhrbeinen . . . . . . . . . 26

8. Morphologische Asymmetrie der Röhrbeinpaare . . . . . . . . . . . . . 26

Prüfung der Knochen auf ihre Widerstandsfähigkeit . . . . . . . . . . . . . . . 27

1. Prüfung der Biegefestigkeit . . . . . . . . . . . . . . . . . . . . . 30

2. Bruchformen . . . . . . . . . . . . . . . . . . . . . . . 31

3. Die Tragfähigkeit . . . . . . . . . . . . . . . . . . . . . . 32

4. Die Durchbiegung der Knochen . . . . . . . . . . . . . . . . . . . . 34

5. Asymmetrie der Leistung . . . . . . . . . . . . . . . . . . . . . . . 39

6. Vergleich nur der stärkeren Knochen der einzelnen Röhrbeinpaare . . • . 39

7. Korrelation von Form und Leistung . . . . . . . . . . . . . . . . . 40

8. Ursachen der unregelmässigen Widerstandsfähigkeit . . . . . . . . . . . 41

9. Torsionsversuche . . . . . . . . . . . . . . . . . . . . . . . . . 44

Zusammenfassung der Resultate:

A. Die Form betreffend . . . . . . . . . . . . . . . . . . . . . . . 46

B. Die Leistung betreffend . . . . . . . . . . . . . . . . . . . 47 



\section{Einfuihrung.}

Immer wieder stösst man in der hippologischen Literatur auf die Behauptung, dass die Knochen der Laufpferde ron festerem Gefüge seien als die der Schrittpferde. Man spricht sogar von einer "elfenbeinartigen“ Beschaffenheit der Laufpferdeknochen gegenüber den gewöhnlichen „porösen“ Knochen der Schrittpferde. Wann dieses Vorurteil einmal entstanden ist, wer die Priorität dafür beanspruchen kann, wird wohl nie nachgewiesen werden. Aber es ist da. Der Verehrer des „edlen“ Pferdes ist fest davon überzeugt, das Publikum sprichts nach, und selbst Bücher und Schriften, die objektiv sein wollen, stellen die grössere Widerstandsfähigkeit der Knochen der Laufpferde so ohne Einschränkung hin, als ob sie durch exakte Versuche bewiesen wäre. Manchem ist das dann ein willkommenes Mittel, die Laufpferde als „edle“ den „gemeinen“ gegenüberstellen zu können und damit womöglich die ganze Zucht des Schrittpferdes zu diskreditieren. Aber die häufigeren Knochenbrüche der Laufpferde hat man damit doch nicht hinwegdisputieren können.

$\mathrm{Ob}$ nun wirklich die Struktur der Knochen bei Lauf- und Schrittpferden eine verschiedene ist, kann schliesslich gleichgültig sein, wenn beide nur eine genügende Festigkeit besitzen. Erweist sich die Widerstandsfähigkeit der Schrittpferdeknochen als geringer wie die der Knochen der Laufpferde, so ist auch die Struktur geringwertiger, und man wird diesen Nachteil durch Anzucht möglichster Starkknochigkeit zu kompensieren suchen müssen. Ist die Brüchigkeit des Laufpferdeknochens eine grössere, so kann diese entweder durch eine zu geringe Stärke (Dicke) der Knochen bedingt sein, oder aber die gerühmte „elfenbeinartige" Struktur ist der Grund für eine grössere Sprödigkeit. Nun, dann ist diese Struktur eben ein Fehler, aber kein Vorzug, als welcher sie so gern hingestellt wird.

Um zur Klarlegung dieser so oft gestreiften Frage beizutragen, stellte ich mir die Aufgabe, die mechanische Widerstandsfähigkeit der Knochen von Lauf- und Schrittpferden zahlenmässig zu prüfen; "denn“, sagt Nehring, ${ }^{1}$ ) „die Lehre vom Exterieur erhält erst dann eine wissenschaftliche

$\left.{ }^{1}\right)$ Nehring, Fossile Pferde aus deutschen Diluvialablagerungen. 
Basis, wenn sie auf genaue osteologische Messungen begründet wird; exakte Messungen haben dauernden Wert, während allgemeine Betrachtungen ohne gleichzeitige Mitteilung absoluter Zahlen einen solchen nicht beanspruchen können".

Meine Untersuchungen mussten sich auf drei rerschiedene Arbeiten erstrecken: Zuerst die Auswahl und das Messen der lebenden Pferde, dann die Auswahl und das Messen der Knochen und zuletzt die Prüfung der Knochen auf ihre Widerstandsfähigkeit.

Ich konnte meine Untersuchungen nur an einem Orte ausführen, an dem ich geeignete Pferde in genügender Zahl fand, um sie lebend messen und nach ihrer Schlachtung die betr. Knochen entnehmen zu können. Gleichzeitig musste die Möglichkeit gegeben sein, die Knochen sofort auf ihre Festigkeit zu untersuchen. Beide Bedingungen waren in Berlin erfüllt. Das Pferdematerial war auf der Zentralrossschlächterei zu finden und die Biegeversuche liessen sich mit peinlichster Exaktheit auf dem Königlichen Material-Prüfungsamt in Gross-Lichterfelde ausführen.

Durch das Entgegenkommen des Herrn Kreistierarztes Dr. Klaus und des Herrn Dr. HöPfNer war es mir ermöglicht, auf der Zentralrossschlächterei die Pferde für meine Versuche zu benutzen. $\mathrm{Zu}$ besonderem Danke aber bin ich Herrn Prof. Rudeloff rom Königlichen MaterialPrüfungsamt der Technischen Hochschule rerpflichtet, der mir nicht nur gestattete, die dortigen grossartigen Eimrichtungen für meine Untersuchungen zu benutzen, sondern mir auch durch seine fachwissenschaftlichen Ratschläge die Durchführung meiner Arbeit ermöglichte. So konnte ich im Frühjahr 1905 meine Untersuchungen ausführen.

\section{Das Pferdematerial.}

Um zu einem deutlichen Resultat zu kommen, entschied ich mich für zwei extreme Rassen, nämlich für das belgische und das ostpreussische Pferd, oder besser gesagt - da die Herkunft der Pferde doch nur selten mit Sicherheit festzustellen ist - für Pferde im belgischen und im ostpreussischen Typus. Von beiden konnte ich annehmen, am ehesten eine genügende Anzahl für meine Versuche zu finden; sonst wäre mir englisches oder arabisches Vollblut und seine nahen Kreuzungen selbstrerständlich lieber gewesen, schon um dem eventuellen Vorwurf entgehen zu können, dass das ostpreussische Pferd doch noch nicht „edel" genug für solche vergleichenden Versuche wäre.

Auf der Zentralrossschlächterei werden täglich ca. 40-60 Pferde geschlachtet. Es sind das fast nur solche, bei denen der Fleischwert dem Gebrauchswert mindestens gleichkommt, meist wird er ihn sogar übersteigen. Es gehört sogar zu den Ausnahmen, wenn ein Besitzer sein noch arbeitsfähiges Pferd lieber für geringes Geld an den Rossschlächter verkauft, ehe er es im Alter in schlechte Hände kommen lässt. Meist wird diese Rücksicht nur auf Reitpferde, seltener auf Wagenpferde genommen; aber nur einmal habe ich ein Lastpferd auf dem Schlachthof gesehen, dem man diese 
Wohltat erwies. Diese Umstände bedingen es, dass jüngere Pferde selten auf dem Schlachthof zu finden sind. Die meisten befinden sich in einem Alter, das man an den Zähnen nicht mehr mit Sicherheit bestimmen kann. Jüngere Pferde kamen nur dann zur Schlachtung, wenn aus irgend einem Grunde, meist Beinbruch, eine Notschlachtung nötig war. Dann war aber eine Messung selten auszuführen und das Pferd war für meine Versuche verloren. Einmal aber liess ein Pferdehändler seinen ganzen Pferdebestand, die Nummern L 20 bis L 34, schlachten, da die Möglichkeit einer Rotzinfektion vorlag. Das waren alles Laufpferde, meist jüngere, und so kommt es, dass unter den 100 Pferden, deren Knochen ich zu meinen Versuchen benutzte, doch noch 17 Laufpferde, aber nur 4 Schrittpferde sind, deren Alter unter 10 Jahren war. Im ganzen liess sich also noch bei 21 Pferden das Alter mit Sicherheit bestimmen. Unter diesen sind 7 neunjährige, 5 achtjährige, 2 siebenjährige, 1 sechsjähriges, 3 fünfjährige und 2 vierjährige. Von den hin und wieder erscheinenden Fohlen habe ich nur 1 einjähriges vergleichsweise für meine Arbeit benutzt. Das Durchschnittsalter der untersuchten Laufpferde war, soweit es sich eben bestimmen liess, 12 Jahre, das der Schrittpferde 13 Jahre.

Auffallend war der geringe Prozentsatz von Schrittpferden und darunter besonders der typisch belgischen Form, die mir mit der grossen Zahl der in Berlin tätigen Belgier gar nicht harmonieren wollte. Eine Erklärung hierfür mag sein, dass ein vollfleischiger Belgier die beste Schlachtware darstellt, für welche in Berlin nicht genügend Nachfrage herrscht, da hier das Pferdefleisch noch nicht so gewürdigt wird, wie in Westdeutschland. Es werden deshalb - ebenso wie Berliner Händler die leichten Klepper in Posen und Ostpreussen für den Berliner Schlachthof heranschaffen auch rheinische Händler die schweren Schlachtpferde aus der Mark, selbst aus Berlin für die rheinischen Rossschlächtereien aufkaufen. Auch müssen die Rossschlächter der Provinzstädte, deren Kundschaft die zur Schlachtung kommenden Pferde kontrollieren kann, weit mehr auf gute Schlachtware sehen, als das in Berlin der Fall ist, so dass wohl eine grosse Zahl der Berliner Belgier in die Provinz geht. Ein zufälliger Umstand verhinderte noch weiter die Zuführung schwerer Pferde. Das war die grosse Hitze, welche im Juli 1905 eintrat und die Aufbewahrung so voluminöser Fleischmassen, wie die Belgier sie liefern, erschwerte. Das hielt von der Schlachtung der schweren Pferde ab, auch deshalb, weil die Hitze den Fleischkonsum herabdrückte. An manchen Tagen wurde nicht ein einziges brauchbares belgisches Pferd auf den Schlachthof gebracht. Dadurch sah ich mich genötigt, meine Untersuchungen abzubrechen, ehe ich die gewünschte Zahl von Belgiern geprüft hatte. Um aber die Zahl der Schrittpferde nicht gar zu sehr hinter der der Laufpferde zurückstehen zu lassen, habe ich auch einige Pferde englischer und dänischer Form für meine Untersuchungen benutzt.

Weniger Schwierigkeiten machte die Auswahl der Laufpferde; aber in meiner Hoffnung, meist solche mit Brand benutzen zu können, wurde 
ich sehr enttäuscht. Ich werde auf dem Schlachthof während meiner dortigen Arbeit ca. 1500 Pferde gesehen haben; aber unter diesen fand ich alles in allem etwa 1 Dutzend mit Brand, Zucht- oder Militärbrand. So war ich denn gezwungen, nach freiem Urteil zu wählen. Da unter den kleineren Pferden häufig sehr edle Formen waren, so habe ich auch mehrere im Stil des leichten Husarenpferdes für meine Untersuchungen benutzt, vergleichsweise auch eins von nur $1,33 \mathrm{~m}$ Widerristhöhe.

Da es möglich ist, dass die Festigkeit der Knochen der Wallache eine andere ist als die der Stuten, so wollte ich meine Untersuchungen hauptsächlich auf Stuten beschränken. Da trat mir bei den Belgiern eine neue Schwierigkeit entgegen: es waren in der grossen Mehrzahl Wallache. Das lässt sich wohl nur so erklären, dass man in Belgien die Stuten mehr zu Zuchtzwecken zurückhält und in der Hauptsache Wallache ausführt. Ausserdem nimmt der Konsument, bei dem, wie in Berlin naturgemäss, die Benutzung zur Zucht unmöglich ist, lieber Wallache. So kommt es, dass ich unter den 60 Laufpferden ausser dem Hengst L 1 nur 10 Wallache, unter den 38 Belgiern aber 22 Wallache aufnehmen musste.

Während meiner dortigen Tätigkeit wurde nur 1 Hengst geschlachtet, ein Ostpreusse des Zirkus Schumann. Leider geschah dies, während ich in Lichterfelde beschäftigt war, so dass ich ihn lebend nicht mehr zu sehen bekam und ihn deshalb auch nicht messen konnte. Seine Vorderröhren habe ich aber mit geprüft.

\section{Das Messen der Pferde.}

Die Messungen habe ich nach Prof. S. v. NAthusius ${ }^{1}$ ) ausgeführt, habe mich aber auf die wichtigsten Mafse beschränkt. Gar zu gern hätte ich auch das Gewicht der Pferde festgestellt, um dann das Verhältnis der Widerstandsfähigkeit der Knochen zu der von ihnen zu tragenden Last prüfen zu können. ${ }^{2}$ ) Aber auf der Berliner Zentralrossschlächterei befindet sich keine Wage, auf der man ein Pferd lebend oder tot wiegen könnte; auch ist die Aufstellung einer solchen dort wegen des äusserst beschränkten Raumes nicht möglich. Die Pferde aber vom Hof herunterzunehmen und auf einer Wage in der Nachbarschaft zu wiegen, liess sich wegen der veterinär-polizeilichen Kontrolle nicht durchführen. So musste ich leider auf die Feststellung des Gewichtes verzichten. Ich versuchte, mir durch Schätzung des Gewichtes zu helfen, wobei ich von Praktikern unterstützt wurde. Aber die einzelnen Annahmen gingen meist so weit auseinander, dass ich schliesslich darauf verzichtete. Auch wurde mir gesagt, dass es weit schwerer sei, ein Pferd nach dem Gewichte zu schätzen, als ein

1) S. v. Nathusius, Unterschiede zwischen der morgen- und abendländischen Pferdegruppe. Verlag von Paul Parey in Berlin. 1891.

2) Auf die Berechtigung dieser Gegenüberstellung von Knochenstärke und Pferdegewicht gehe ich noch näher im Abschnitt über die „typische Belastung der Röhrenknochen", Seite 17, ein. 
anderes Schlachttier. Man wollte dies durch das geringere spezifische Gewicht des Pferdefleisches begründen. Noch mehr mag dazu aber beitragen der verschieden starke Knochenbau und die sehr variierende Form des Pferdekörpers, besonders wohl Mangel an Übung, da der Pferdeschlächter bei den geringen Preisen für Schlachtpferde kein grossen Interesse an genauer Gewichtsfeststellung hat.

Die Ausführung der Messungen machte weiter keine Schwierigkeiten, da auch den unruhigsten der dortigen Pferde der nötige Lebensmut fehlte. Nur war es öfter unmöglich, die für die Messungen ausersehenen Pferde aus dem gemeinsamen Stall, in welchem sie ganz frei, selbst ohne Halfter, dichtgedrängt standen, oft enger wie die Schafe im Stall, herauszufinden. Dadurch ging mir noch manches Pferd für meine Untersuchungen verloren.

Im ganzen habe ich ca. 150 Pferde gemessen und von diesen die Knochen entnommen. Viele Knochen waren aber durch Exostosen derart deformiert, dass ich sie für meine Versuche nicht verwenden konnte. Ich habe dann nicht nur den unbrauchbaren Knochen, sondern auch den dazu gehörigen verwerfen müssen. - Oft war ich mir trotz aller Aufmerksamkeit nicht sicher, auch wirklich die Knochen von dem gemessenen Pferde und nicht statt deren die eines anderen bekommen zu haben. Auch dann habe ich lieber die Knochen verworfen. - Stellte sich dann schliesslich bei der Verrechnung der durch Messung gewonnenen Zahlen heraus, dass eine dieser Zahlen nicht richtig sein konnte, so habe ich diese Zahl nicht korrigiert, sondern das betreffende Knochenpaar ausgeschieden. So sind schliesslich von den 150 entnommenen Knochenpaaren nur 99 übrig geblieben. Hiervon sind 61 Laufpferde und 38 Schrittpferde. Zur bequemeren Orientierung habe ich den Nummern der Laufpferde den Buchstaben L, den Nummern der Schrittpferde den Buchstaben S vorgesetzt. Es führen demnach die Laufpferde die Nummern L 1 bis L 61, die Schrittpferde die Nummern S 1 bis S 38. - L 60 ist das einjährige Fohlen und L 61 das kleine, 1,33 $\mathrm{m}$ hohe Pferd, welche beide nur vergleichsweise aufgeführt wurden. Thre Zahlen sind zur Gewinnung der Durchschnittszahlen niemals mitverwandt.

Die Malse dieser 99 Pferde habe ich als ersten Block in der Haupttabelle, nach den beiden Schlägen geordnet, zusammengestellt. In der Tabelle II gebe ich die daraus berechneten Durchschnittszahlen.

Nehmen wir die Zahlen, welche S. v. Nathusius in den „Messungen an Hengsten, Stuten und Gebrauchspferden" 1 ) angibt, als die normalen an und vergleichen damit die Zahlen unserer Tabelle II, so kommen wir zu folgendem Ergebnis: Die zu den vorliegenden Versuchen benutzten Belgier entsprechen im Durchschnitt in der Widerristhöhe den Original-Belgiern, die Laufpferde aber erreichen im Mittel nicht die Durchschnittsgrösse der deutschen Edelzuchten, sie sind etwa $1 \mathrm{~cm}$ kleiner. Wichtiger sind die relativen Zahlen für die übrigen Mafse. Diese sind durchweg geringer als

1) Heft 112 der "Arbeiten der Deutschen Landwirtschafts-Gesellschaft". - Die dort gegebenen Zahlen sind in Tabelle II in Klammern beigefügt. 
die für die betreffenden Schläge von S. v. Nathusius gefundenen und beweisen die Minderwertigkeit des von mir benutzten Materials, wobei allerdings berücksichtigt werden muss, dass S. v. NAthusius meist Zuchttiere gemessen hat, welche ja immer die Elite darstellen, und dazu meist in Ausstellungskondition vorgeführt sind, während die zu vorliegenden Untersuchungen benutzten Pferde im Durchschnitt sich in einem kaum mässigen Ernährungszustande befanden. Dies tritt besonders dadurch in die Erscheinung, dass gerade der Brustumfang der untersuchten Pferde am meisten zu wünschen übrig lässt, und „das ist gerade das Mafs, welches mehr wie jedes andere durch den Futterzustand beeinflusst wird". Die relative Rumpflänge entspricht fast genau den Zahlen, welche S. v. Nathusıus für die Gebrauchspferde ermittelt hat, und welche um 3-5\% hinter der Rumpflänge der Zuchtpferde zurückstehen. Der relative Röhrbeinumfang ist bei den Lauf- und Schrittpferden normal, ebenso Kruppenhöhe und Brusttiefe der Laufpferde. Dass die Mafse der untersuchten Schrittpferde mehr schwankten als die der Laufpferde, lässt sich dadurch erklären, dass, wie schon erwähnt, verschiedene Schrittpferdeformen bei den Untersuchungen benutzt werden mussten.

\section{Die Auswahl der Knochen.}

Für die Auswahl der Knochen waren zwei Punkte entscheidend: Erstens mussten sie so frei liegen, dass man sie schon am lebenden Tiere möglichst auf ihre Stärke beurteilen kann; zweitens musste ihre Stärke von Einfluss auf die Leistungsfähigkeit des Pferdes sein. Demnach standen mir zur Wahl die vorderen und die hinteren Röhrbeine. Prof. L. HofrmansStuttgart hatte bei ähnlichen Versuchen ${ }^{1}$ ) die hinteren Röhrbeine gewählt, und zwar, weil diese die „widerstandsfähigsten von sämtlichen LängsExtremitätenknochen" des Pferdes seien. Ich entschied mich für das vordere Röhrbein, den Metacarpus, einmal weil dieser meist schon am lebenden Pferde zur Beurteilung der Knochenstärke dient, wie ja auch S. v. NAthusius bei seinen Pferdemessungen das vordere Röhrbein wählte. Dann aber ist auch zu erwägen, dass der widerstandsfähigere Knochen dem Brechen doch weniger ausgesetzt ist. Daher muss es wichtiger erscheinen, die Festigkeit des schwächeren, also des gefährdeteren Knochens zu prüfen. Das ist das vordere Röhrbein.

\section{Das Messen der Knochen.}

Ich hatte mich zunächst darüber zu entscheiden, ob ich mit dem eigentlichen Röhrbein allein, also dem Metacarpus III, operieren wollte, oder ob das Röhrbein samt den beiden Griffelbeinen, also Metacarpus II, III und IV, als ein Ganzes zu betrachten war. - Fast immer waren die Griffelbeine, wenigstens stellenweise, so innig mit dem Röhrbein verwachsen, dass eine Trennung dieser Knochen ohne Verletzung der Röhre nicht möglich gewesen wäre. Da hierdurch die Tragkraft des Röhrbeins je nach dem Grade der Verwachsung sehr verschieden beeinflusst werden musste,

1) Berliner Tierärztliche Wochenschrift Jahrgang 1901, No. 2. 
so war ich genötigt, die drei Knochen als Ganzes zu untersuchen. Das hat natürlich zur Folge, dass sich bei den Festigkeitsuntersuchungen Zahlen ergeben müssen, welche je nach dem Grade der Verwachsung und der Stärke der Griffelbeine die Widerstandsfähigkeit des isoliert gedachten Röhrbeins mehr oder weniger übersteigen, und zwar so, dass die für den Metacarpus III faktisch geltenden Zahlen durchaus nicht äquivalente Teile der durch meine Versuche gewonnenen Zahlen sind. Das mag ja den Wert der vorliegenden Untersuchungen beeinträchtigen; aber es ist doch auch nicht zu übersehen, dass die Widerstandsfähigkeit der Röhrbeine bei lebenden Tieren durch die eben geschilderte Unterstützung der Griffelbeine auch gewinnt, so dass man die Prüfung der Röhrbeine inkl. der Griffelbeine für die den natürlichen Verhältnissen mehr entsprechende, also richtigere halten kann. Doch stehe ich nicht an zu erklären, dass mich nur die schwierige Abtrennung der Griffelbeine davon zurückgehalten hat, das isotierte Röhrbein zu prüfen, weil doch die Untersuchung einfacherer Verhältnisse möglichst der der komplizierteren vorangehen sollte.

Das Belassen der Griffelbeine am Röhrbein hatte einen weiteren Übelstand zur Folge. Da, um die Beschaffenheit der Knochenmasse nicht zu verändern, bei der Reinigung der Knochen von der Benutzung chemisch wirkender Substanzen, selbst vom Kochen abgesehen werden musste, so gestaltete sich diese Arbeit wegen der enganliegenden Griffelbeine sehr schwierig, und es war absolut unmöglich, die Weichteile zwischen Griffelbein und Röhrbein, besonders am proximalen Fnde, vollständig zu entfernen. So wurde ich verhindert, die Wägungen der Knochen mit der sonst wünschenswerten Genauigkeit auszuführen. Dadurch wurden berührt die Zahlen für das absolute Gewicht, für das Volumen und für das spezifische Gewicht. Ich musste mich hier mit einer Genauigkeit von $1 / 2 g$ begnügen, die aber wohl genügen dürfte.

Die Knochenmalse habe ich in der Haupttabelle gleich unter den Pferdemalsen aufgeführt. Jedoch musste ich für die auf den Querschnitt bezüglichen Mafse spezielle Tabellen anlegen, um die Haupttabelle nicht zu überfüllen.

\section{Das absolute Gewicht.}

Das absolute Gewicht schwankte bei den Laufpferden von $353 g$ (No. L 21 l.) bis $589 g$ (No. L 58 r.) und betrug im Mittel 450,44 g. Bei den Schrittpferden variierte es von $453 g$ (No. S 12 1.) bis $726 g$ (No. S 25 r.). Der Durchschnitt war 588,95 $\mathrm{g}$.

\section{(Siehe die Tabelle auf Seite 8.)}

Wenn auch in einzelnen Fällen das Gewicht des rechten und linken Röhrbeins deutlich differierte (bei den Laufpferden bis zu 6,5\% bei No. L 38, bei den Schrittpferden bis zu $6,6 \%$ bei No. S 34), so verschwindet dieser Unterschied beim Durchschnitt fast ganz. Es betrug im Mittel bei Laufpferden das Gewicht der linken Röhre 450,7 g, das der rechten 450,2 $\mathrm{g}$. Bei Schrittpferden waren die entsprechenden Zahlen 588,8 und 589,2 $\mathrm{g}$. 
Hierbei ist nicht zu übersehen, dass bei diesen Gewichtsangaben die Griffelbeine eingeschlossen sind. - Schon hier habe ich den Mangel der Gewichtszahlen für das lebende Pferd empfunden. Ich hätte auf Grund dieser Zahlen gern das Verhältnis zwischen Röhrbeingewicht und Lebendgewicht bei Laufund Schrittpferden festgestellt. Dasselbe gilt auch von dem folgenden.

Reihenfolge der Lauf- und Schrittpferde, geordnet nach dem absoluten Gewicht nur des stärkeren Metacarpus jedes Paares.

\begin{tabular}{|c|c|c|c|c|c|c|c|c|c|}
\hline \multicolumn{6}{|c|}{ L a u f p ferde: } & \multicolumn{4}{|c|}{ Schrittpferde: } \\
\hline $\begin{array}{c}\text { No. } \\
\text { L }\end{array}$ & $\begin{array}{c}\text { Absolutes } \\
\text { Gewicht } \\
\text { in } g\end{array}$ & $\begin{array}{c}\text { No. } \\
\text { L }\end{array}$ & $\begin{array}{c}\text { Absolutes } \\
\text { Gewicht } \\
\text { in } g\end{array}$ & $\begin{array}{c}\text { No. } \\
\text { L }\end{array}$ & $\begin{array}{c}\text { Absolutes } \\
\text { Gewicht } \\
\text { in } g\end{array}$ & $\begin{array}{c}\text { No. } \\
\text { S }\end{array}$ & $\begin{array}{c}\text { Absolutes } \\
\text { Gewicht } \\
\text { in } g\end{array}$ & $\begin{array}{c}\text { No. } \\
\text { S }\end{array}$ & $\begin{array}{c}\text { Absolutes } \\
\text { Gewicht } \\
\text { in } g\end{array}$ \\
\hline 21 & 359 & 15 & 425 & 47 & 476 & 12 & 460,5 & 38 & 599 \\
\hline 36 & 366,5 & 26 & 429 & 57 & 476 & 32 & 465 & 16 & 603 \\
\hline 34 & 376 & 30 & 430 & 38 & 486 & 1 & 472 & 15 & 605,5 \\
\hline 52 & 381,5 & 55 & 430,5 & 27 & 488 & 27 & 501,5 & 35 & 606,5 \\
\hline 42 & 382 & 6 & 434,5 & 54 & 490 & 14 & 517 & 8 & 610,5 \\
\hline 43 & 387,5 & 22 & 437 & 59 & 493 & 7 & 517,5 & 2 & 612,5 \\
\hline 24 & 390 & 37 & 438 & 13 & 495,5 & 17 & 535 & 29 & 616 \\
\hline 10 & 397 & 39 & 438 & 16 & 498 & 28 & 540 & 26 & 617 \\
\hline 2 & 397,5 & 3 & 441 & 51 & 501,5 & 31 & 542 & 33 & 627 \\
\hline 35 & 398 & 32 & 443 & 46 & 503,5 & 30 & 547,5 & 11 & 633 \\
\hline 14 & 403 & 4 & 444 & 28 & 505 & 21 & 551 & 23 & 640 \\
\hline 19 & 406 & 40 & 445 & 17 & 521 & 10 & 559 & 13 & 645,5 \\
\hline 11 & 406 & 25 & 445,5 & 7 & 527 & 22 & 559 & 6 & 651,5 \\
\hline 29 & 409 & 5 & 446 & 31 & 546 & 20 & 563 & 36 & 666 \\
\hline 20 & 413 & 9 & 447 & 41 & 546 & 34 & 568 & 9 & 676,5 \\
\hline 44 & 416 & 56 & 457,5 & 23 & 572 & 5 & 568,5 & 18 & 689 \\
\hline 8 & 420 & 49 & 459,5 & 58 & 583 & 24 & 569 & 4 & 699 \\
\hline 33 & 422 & 48 & 472,5 & 18 & 585 & 37 & 581 & 3 & 709,5 \\
\hline 50 & 423 & 1 & 473,5 & 12 & 620,5 & 19 & 593,5 & 25 & 724,5 \\
\hline 53 & 423 & 45 & 475 & & & & & & \\
\hline
\end{tabular}

2. Das Volumen.

Dies suchte ich nicht zu bestimmen durch Messung der durch die Knochen verdrängten Wassermenge, sondern ich stellte den Gewichtsverlust fest, welchen der Knochen beim Wiegen im Wasser erleidet. Das beruht allerdings auf demselben Prinzip, gibt aber genauere Resultate als die erstgenannte Methode. Um den Knochen im Wasser wiegen zu können, legte ich ihn wagerecht in zwei dünne Drahtschlingen. Vorher wurde er mit einem ins Wasser getauchten Pinsel sorgfältig bestrichen, um das Anhaften von Luftblasen zu verhindern. Diese hätten sein Gewicht im Wasser geringer, seinen Gewichtsverlust und also auch sein Volumen grösser, sein spezifisches Gewicht wieder kleiner erscheinen lassen. Der Gewichtsverlust, den die beiden Drahtschlingen im Wasser erlitten, wurde nicht berïcksichtigt; er kam bei der Feinheit des Drahtes und neben der durch die nicht entfernten Weichteile verursachten geringen Ungenauigkeit nicht zur Geltung. 
Das Volumen betrug bei den Laufpferden im Mittel 283,7 ccm und war am geringsten bei No. L 21 l. mit $220 \mathrm{ccm}$, am höchsten bei No. L $12 \mathrm{r}$. mit $388,5 \mathrm{ccm}$. Die Schrittpferde zeigten ein Durchschnittsvolumen von $371,8 \mathrm{ccm}$ und schwankten von $281 \mathrm{ccm}$ (No. S $13 \mathrm{r}$.) bis zu $457 \mathrm{ccm}$ bei No. $\mathrm{S} 3 \mathrm{r}$.

Reihenfolge der Lauf- und Schrittpferde, geordnet nach dem Volumen nur des stärkeren Metacarpus jedes Paares.

\begin{tabular}{|c|c|c|c|c|c|c|c|c|c|}
\hline \multicolumn{6}{|c|}{ La ufpferde: } & \multicolumn{4}{|c|}{ Schrittpferde: } \\
\hline $\begin{array}{c}\text { No. } \\
\text { L }\end{array}$ & $\begin{array}{l}\text { Volumen } \\
\text { in } \mathrm{ccm}\end{array}$ & $\begin{array}{c}\text { No. } \\
\mathrm{L}\end{array}$ & $\begin{array}{l}\text { Volumen } \\
\text { in } \mathrm{ccm}\end{array}$ & $\begin{array}{c}\text { No. } \\
\text { L }\end{array}$ & $\begin{array}{l}\text { Volumen } \\
\text { in } c c m\end{array}$ & $\begin{array}{c}\text { No. } \\
\text { S }\end{array}$ & $\begin{array}{l}\text { Volumen } \\
\text { in } \mathrm{ccm}\end{array}$ & $\begin{array}{c}\text { No. } \\
\text { S }\end{array}$ & $\begin{array}{c}\text { Volumen } \\
\text { in } c c m\end{array}$ \\
\hline 36 & 221,5 & 50 & 262,5 & 38 & 297,5 & 12 & 281,5 & 38 & 373,5 \\
\hline 21 & 222,5 & 4 & 265 & 47 & 299 & 32 & 296 & 19 & 374 \\
\hline 42 & 232,5 & 55 & 266,5 & 13 & 300 & 1 & 300 & 2 & 374,5 \\
\hline 43 & 232,5 & 15 & 268 & 27 & 303 & 27 & 307,5 & 26 & 376,5 \\
\hline 24 & 233,5 & 32 & 270 & 54 & 303 & 14 & 322 & 35 & 381 \\
\hline 35 & $24 \check{5}, 5$ & 37 & 270 & 16 & 307,5 & 21 & 333 & 16 & 382,5 \\
\hline 10 & 246 & 8 & 273 & 48 & 309 & 30 & 333,5 & 15 & 388 \\
\hline 52 & $247,5^{\bullet}$ & 22 & 273 & 28 & 312,5 & 7 & 337 & 29 & 391 \\
\hline 2 & 250 & 5 & 273,5 & 7 & 314 & 31 & 339 & 33 & 392 \\
\hline 11 & 253 & 6 & 276,5 & 59 & 314,5 & 34 & 341,5 & 13 & 399 \\
\hline 20 & 253 & 25 & 276,5 & 46 & 316,5 & 17 & 342,5 & 23 & 403,5 \\
\hline 34 & 253,5 & 9 & 279,5 & 17 & 319 & 28 & 347 & 11 & 424 \\
\hline 19 & 254 & 39 & 283 & 51 & 329,5 & 10 & 354 & 36 & 424 \\
\hline 29 & 254 & 40 & 283,5 & 41 & 346 & 24 & 354,5 & 9 & 427 \\
\hline 53 & 254 & 3 & 286 & 58 & 346,5 & 5 & 355 & 6 & 427,5 \\
\hline 30 & 257,5 & 1 & 287 & 31 & 350 & 22 & 355 & 18 & 438,5 \\
\hline 14 & 258 & 45 & 289 & 23 & 357 & 20 & 362 & 4 & 445 \\
\hline 33 & 259 & 56 & 290,5 & 18 & 370 & 37 & 362 & 25 & 449 \\
\hline 26 & 261 & 49 & 291 & 12 & 388,5 & 8 & 371,5 & 3 & 450 \\
\hline 44 & 262 & 57 & 293,5 & & & & & & \\
\hline
\end{tabular}

Auch beim Volumen ergaben sich einige erhebliche Abweichungen zwischen dem rechten und linken Röhrbein. Diese waren, wie auch die Gewichtsdifferenz, bei Schrittpferden am grössten bei No. S 34, wo sie zugunsten von links $5,9 \%$ betrug. Im Durchschnitt betrug das Volumen links 370,8, rechts $372,8 \mathrm{ccm}$, was eine Differenz von nur $0,54 \%$ ergibt. Bei den Laufpferden war kaum ein Unterschied zwischen links und rechts feszustellen. Das Durchschnittsvolumen betrug links 283,7, rechts 283,8 ccm, doch zeigten sich bei einzelnen Pferden Differenzen bis zu 7\% (No. L 51).

\section{Das spezifische Gewicht.}

Bei der Genauigkeit der Wägungen von $1 / 2 g$ konnte das spez. Gewicht mit genügender Sicherheit auf zwei Dezimalstellen berechnet werden. Es schwankte bei den Laufpferden von 1,49 (No. L 34 l.) bis 1,68 (No. L 4 und L 58) und betrug im Durchschnitt 1,605. Eine Differenz zwischen links und rechts ergab sich bei den Mittelzahlen erst in der vierten Dezimalstelle, obgleich sich wieder in einzelnen Fällen erhebliche Unterschiede 
ergaben, so bei No. L $34=7,4 \%$. Die Schrittpferde zeigten einen Gesamtdurchschnitt von 1,586, rechts einen solchen von 1,585, links von 1,588. Die grösste Differenz zwischen links und rechts wies No. S 7 auf, nämlich $4,6 \%$. Im ganzen schwankte das spez. Gewicht bei den Schrittpferden von 1,49 (No. S 6 r.) bis 1,66 (No. S 34 l.). Im Durchschnitt ist also das spez. Gewicht bei den Schrittpferden etwas geringer als bei den Laufpferden (1,586 gegen 1,605), und zwar um 0,019 oder 1,2\%. Diese Zahlen erscheinen allerdings so klein, dass man geneigt sein könnte, sie ganz unbeachtet zu lassen, also die Knochen der Lauf- und Schrittpferde nach dem spez. Gewicht gleich zu bewerten. Hierbei ist aber folgendes zu beachten. Alle angeführten Zahlen für das spez. Gewicht beziehen sich nicht auf die Knochenmasse an sich, sondern gelten für den Knochen als Ganzes, also inkl. der Markhöhle. Da diese nur durch das spezifisch leichte Mark ausgefüllt ist, so muss in jedem Falle der ganze Knochen spezifisch leichter sein, als die Knochenmasse an sich. Nun ist aber die Markhöhle der Schrittpferde, wie sich auch bei meinen Untersuchungen der Wandstärke (Seite 17) erwiesen hat, auch relativ grösser als die der Laufpferde. Das muss sich dahin äussern, dass die Differenz zwischen dem spez. Gewicht des ganzen Knochens und dem der eigentlichen Knochenmasse bei den Schrittpferden grösser ist als bei den Laufpferden. Dies ist der Grund dafür, dass das spez. Gewicht der Schrittpferderöhren nach meinen Untersuchungen etwas kleiner ist als das der Laufpferderöhren. Wollte man die berechnete geringe Differenz nur als eine zufällige gelten lassen, so würde man damit ein grösseres spez. Gewicht der eigentlichen Knochenmasse der Schrittpferdeknochen zugeben. Dies hätte sich vielleicht durch Gewichts- und Volumenbestimmungen an äquivalenten Knochenringen deutlich nachweisen lassen. Leider konnte ich das an meinem Knochenmaterial nicht ausführen. Durch die Biegeversuche war zu oft eine Zersplitterung der Knochen verursacht, wobei auch Stücke verloren gingen, so dass lückenlose Knochenringe durch ein Wiederzusammenfügen der einzelnen Bruchstücke nur selten hätten gewonnen werden können.

Die allgemein verbreitete Annahme, die Knochen der Laufpferde seien spezifisch schwerer als die der Schrittpferde, woraus dann weiter auf eine grössere Härte der Laufpferdknochen geschlossen wird, habe ich nicht bestätigt gefunden. Nach mikroskopischen Untersuchungen DETTWEILERS an Knochenschliffen ${ }^{1}$ ) scheinen ja auch nennenswerte Rassenunterschiede in der Knochenstruktur nicht zu bestehen. Die Frage aber, wodurch nun die bedeutende individuelle Verschiedenheit im spez. Gewicht und Bau der Knochen verursacht wird, ist noch offen. Wahrscheinlich spielen dabei neben der Abstammung die Ernährung des Pferdes und die Beanspruchung des Knochens eine Rolle. Fraglich bleibt nur, ob und wie weit wir einst imstande sein werden, die Qualität der Knochen durch Berücksichtigung obiger Faktoren zu beeinflussen.

1) Deutsche landw. Tierzucht, Jahrgang 1904, No. 27. 


\section{Die Länge der Röhren.}

Die Länge der Röhren wurde mittels einer parallel zur Längsachse des Knochens angelegten Schublehre gemessen. Am distalen Ende lieferte immer die mittlere Rippe der Gelenkrolle den Fixpunkt. Am proximalen Ende war es meist die Spitze, in welcher die beiden vorderen Ränder der durch das Hamatum und Capitatum gebildeten Gelenkflächen zusammenstossen; doch war in einigen Fällen die Spitze an der volaren Seite dieser Gelenkflächen der am weitesten hervortretende Punkt. Wegen der relativ beträchtlichen Grösse dieses Mafses glaubte ich mich hier mit einer Genauigkeit von $1 / 2 \mathrm{~mm}$ begnügen zu können.

Bei den Laufpferden beträgt die Länge im Nittel $254,3 \mathrm{~mm}$, und der Durchschnitt von links und rechts stimmt genau überein, während sich bei einzelnen Pferden Differenzen bis zu 1,2\% zeigen (No. L 13). Bei den Schrittpferden ist das linke Röhrbein im Nittel 257,1, das rechte $257 \mathrm{~mm}$ lang, so dass sich hier ein Gesamtdurchschnitt von 257,05 $\mathrm{mm}$ ergibt, der den der Laufpferde um 3,2 $\mathrm{mm}$ übertrifft. Die grösste Differenz zwischen links und rechts zeigt hier No. S 17 mit 1,6\%.

Dass die absolute Länge der Schrittpferd̄eröhrbeine diejenige der Laufpferderöhren um etwas übertrifft, hat seinen Grund in der absolut beträchtlicheren Grösse der Schrittpferde. Vergleicht man die Röhrbeinlänge mit der Widerristhöhe, $\mathrm{L}=159 \mathrm{~cm}, \mathrm{~S}=162 \mathrm{~cm}$, so ergibt sich für die Schrittpferde ein Durchschnitt von $15,89 \%$ gegenüber $16,00 \%$ bei den Laufpferden. Dieser Unterschied kommt auch dadurch dentlich zum Ausdruck, dass die betr. Zahlen bei den Schrittpferden zwischen 14,5 (No. S 24) und 16,5 (No. S 2 und 9), bei den Laufpferden zwischen 15,3 (No. L 43) und 17,4 (No. L 18) schwanken, bei beiden also um $2 \%$, doch so, dass die Zahlen bei den Laufpferden deutlich höher liegen (vergl. Tabelle III). Diese Differenz entspricht dem Unterschied in der relativen Beinlänge beider Rassen, $\mathrm{L}=51,96 \%, \mathrm{~S}=51,13 \%$ der Widerristhöhe.

\section{Der Querschnitt des Röhrbeins.}

(Hierzu Tabelle IV L und IV S.)

Der kleinste Querschnitt des Metacarpus liegt nicht genau in der Mitte, sondern etwa einen Finger breit tiefer. Ich wählte für meine Messungen aber doch den durch die Mitte der Längsachse gelegten Querschnitt, weil ich bei den Biegeversuchen hier den Druck einwirken liess.

Bei der Untersuchung der verschiedenen Querschnittmafse konnte ich leider nicht die Methode anwenden, die ich für die beste zur Erlangung genauer Resultate halte. Das ist nämlich folgende: Aus der Nitte des Röhrbeins wird durch zwei auf der Längsachse senkrecht stehende, also parallele Schnitte ein Knochenring von etwa 4-8 cm Höhe herausgenommen. Von diesem wird zunächst Volumen und spez. Gewicht festgestellt. Ersteres durch die Höhe des Ringes dividiert, ergibt den Flächeninhalt des ringförmigen Querschnittes, also der Knochenmasse ohne Markhöhle. Aus diesem ist leicht die durchschnittliche Wandstärke zu berechnen. Füllt man dann 
die Markhöhle durch eine geeignete Substanz so aus, dass diese mit den Rändern des Knochenringes eine Ebene bildet, so findet man leicht das Volumen des Knochenringes inkl. Markhöhle. Hieraus kann nun berechnet werden das Volumen der Markhöhle allein und der Anteil des Markraumes am massir gedachten Knochenausschnitt, ebenso auch der Anteil der Wände. Durch einfaches Umzeichnen liesse sich dann auch die Form des Querschnittes festlegen. Diese Methode konnte ich leider nicht anwenden, weil ja die Knochen zu Biegeversuchen benutzt werden sollten. Ich konnte nur den grossen und den kleinen Durchmesser des ungefähr ellipsenförmigen Querschnittes durch Messung feststellen. Der kleine liegt in der Längsachse des Pferdes, der grosse rechtwinklig dazu. - Um die verschiedenen Umrissformen des Querschnittes festlegen zu können, hatte ich einen Apparat konstruiert, welcher den Umriss mechanisch genau aufzeichnete, ohne dass dazu der Knochen durchsägt werden brauchte. Leider ist ohne meine Schuld der in Auftrag gegebene Apparat erst fertig geworden, als meine Untersuchungen beendet waren. Es war mir nicht einmal möglich, die Grösse des Umfanges in der Mitte der Röhre festzustellen; das wurde durch die Griffelbeine verhindert.

Der grosse und kleine Querschnittdurchmesser wurde mittelst Schublehre auf $1 / 10 \mathrm{~mm}$ genau festgestellt. Bei dem grossen Durchmesser machte das meist keine Schwierigkeiten. Nur hin und wieder war der Knochen gerade in der Mitte durch die Verwachsung mit den Griffelbeinen, besonders mit dem inneren, so aufgetrieben, dass das Mafs nicht zu nehmen war. Bei der Feststellung des kleinen Durchmessers trat ein anderes Hindernis ein: in vielen Fällen war die volare Seite des Metacarpus derart mit flachen, etwa 3-4 mm breiten Längsrippen und Furchen rersehen, dass Mafsschwankungen bis zu $1 \mathrm{~mm}$ entstanden, je nachdem der betreffende Schenkel der Schublehre in eine Furche oder auf eine Rippe gesetzt wurde. Ich habe in solchen Fällen möglichst den Durchschnitt beider Zahlen genommen. Doch konnte das Mafs nicht so zurerlässig werden, wie das des grossen Durchmessers.

Ein Vergleich beider Durchmesser zeigt, dass das Röhrbein der Schrittpferde im Durchschnitt etwas flacher gebaut ist als das der Laufpferde. Schwarznecker hält die breite, abgeflachte Form für die zweckmässigere, ${ }^{1}$ ) müsste also den Schrittpferderöhren den Vorzug geben. Tabelle IV zeigt in Spalte 3 das Verhältnis von kleinem und grossem Durchmesser. Der kleine betrug rom grossen im Mittel bei Schrittpferden $72,55 \%$, bei Laufpferden $76,74 \%$. Dieser Unterschied ist aber lange nicht so gross wie der individuelle, welcher bei beiden Rassen fast gleichgross ist und von 66 bis $85 \%$ schwankt. Fig. 1 reranschaulicht das Verhältnis der Querschnittdurchmesser. Alle vier Schnitte sind in der Zeichnung auf denselben Flächeninhalt gebracht und als reine Ellipsen aufgefasst. Zwischen den Durchschnittsformen der Lauf- und Schrittpferde ist kaum ein Unterschied zu erkennen. Die indiriduellen Abweichungen sind weit auffallender.

1) Schwarznecker, Pferdezucht Seite 302. 
Zwischen links und rechts bestehen nur geringe Differenzen. Bei Schrittpferden betragen die betr. Zahlen links $72,4 \%$, rechts $72,7 \%$, bei Laufpferden links $76,57 \%$, rechts $76,91 \%$. Bei beiden Rassen erscheint demnach die linke Röhre etwas flacher.

Will man sich aus der Grösse der Durchmesser ein Bild von der Stärke der Röhrbeine machen, so legt man hierbei besser den mittleren Durchmesser zugrunde (siehe Tabelle IV Spalte 4). Dieser schwankt bei den Laufpferden von $28 \mathrm{~mm}$ (No. L 21 l.) bis $37,1 \mathrm{~mm}$ (No. L $12 \mathrm{r}$.) und beträgt im Gesamtdurchschnitt $32,30 \mathrm{~mm}$. Bei den Schrittpferden ist der Durchschnitt $36,91 \mathrm{~mm}$ und das Mafs schwankt von $31,9 \mathrm{~mm}$ (No. S $1 \mathrm{r}$.) bis $41,6 \mathrm{~mm}$ (No. S 4 l.). Die Differenz zwischen linkem und rechtem Röhrbein, die bei einzelnen Paaren immerhin merklich ist, gleicht sich im Durchschnitt fast vollständig aus. Der durchschnittliche mittlere Durchmesser beträgt bei den Laufpferden links 32,22 , rechts $32,30 \mathrm{~mm}$, bei den Schrittpferden links 36,97, rechts $36,85 \mathrm{~mm}$. Beide Röhrenknochen können demnach sowohl bei Laufpferden als bei Schrittpferden im Durchschnitt als gleichstark angenommen werden.

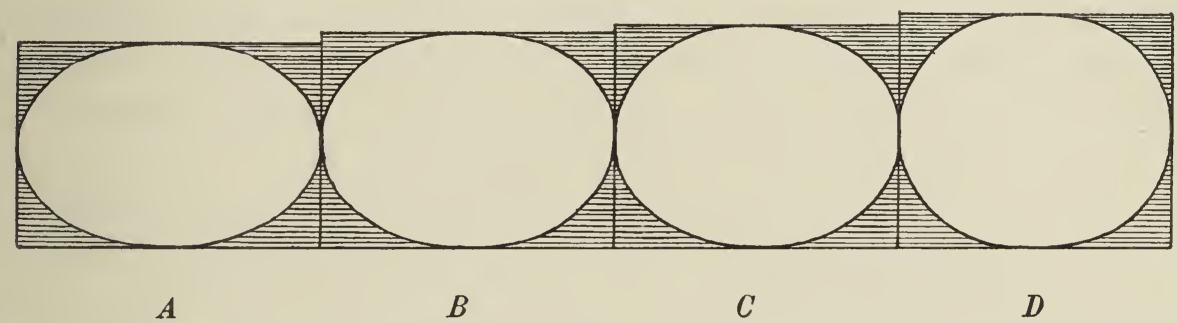

Fig. 1 zeigt das Verhältnis des kleinen zum grossen Durchmesser am Querschnitt des Metacarpus: $B$ im Durchschnitt der Schrittpferde, $C$ im Durchschnitt der Laufpferde, $A$ und $D$ die flachste und höchste Form sowohl bei Lauf- als auch bei Schrittpferden.

Vergleicht man den mittleren Querdurchmesser mit der Länge der Röhrbeine, so ergibt sich bei den Laufpferden für den mittleren Durchmesser $12,71 \%$ der Länge, bei den Schrittpferden 14,36\%. Das Röhrbein der Schrittpferde ist also nicht nur kürzer, sondern hiernach auch gedrungener gebaut als das der Laufpferde, wie das ja auch der Augenschein lehrt. Die Differenzen zwischen links und rechts sind bei beiden Rassen gering. Bei den Laufpferden erscheint das rechte Röhrbein etwas gedrungener $(12,73 \%$ gegen $12,68 \%)$, bei den Schrittpferden das linke $(14,38 \%$ gegen $14,34 \%)$.

Aus den beiden Durchmessern berechnete ich den Flächeninhalt des Querschnittes, den ich zu diesem Zwecke als Ellipse auffasste, obgleich er dieser Form wohl selten ganz entspricht. Die so gewonnenen Zahlen geben natürlich nicht den Querschnitt der wirksamen, tragenden Knochemasse, denn die Markhöhle ist ja bei dieser Berechnungsweise in den Flächeninhalt einbegriffen. Die Zahlen sollen auch nur ein Bild geben von der Stärke (Dicke) des Knochens. Ich fand bei den Laufpferden im grossen Mittel einen Querschnitt von 806 qmm (links 801, rechts 812), bei den Schrittpferden 1047 qmm (links 1050, rechts 1045). 
Denkt man sich innerhalb des Röhrbeins einen Zylinder von der Länge des Knochens konstruiert, welcher den Querschnitt des Knochens zur Grundfläche hat, so liegt ein grosser Teil des Knochenvolumens ausserhalb dieses Zylinders. Diesen Teil des Röhrbeins fand ich bei Lauf- und Schrittpferden auffallend übereinstimmend; er betrug bei beiden genau $27,5 \%$ des ganzen Volumens. Trotzdem hierbei immer die Griffelbeine mit in das Volumen gerechnet sind, darf man diese Übereinstimmung wohl nicht für eine zufällige halten. Dass die Verdickung nach den Enden zu bei den Schrittpferden stärker erscheint, liegt nur daran, dass diese relativ kürzer sind.

Von besonderem Interesse musste eine Gegenüberstellung von Knochenquerschnitt und dem am lebenden Tiere über der Haut gemessenen Röhrbeinumfang sein. Ich habe dazu die Tabelle V L und S aufgestellt. Da am lebenden Pferde immer nur der Umfang des linken Röhrbeines festgestellt wurde, so beziehen sich die Zahlen der Tabelle auch nur auf dieses und den linken Metacarpus. - Es wäre mathematisch korrekter gewesen, den Flächeninhalt beider Querschnitte (des Knochens und den des Mittelfusses über der Haut) zu vergleichen, weil es ja auf die Tragfähigkeit ankommt. Aber in der Praxis ist es üblich, den Röhrbeinumfang als Stärkemals anzugeben. Deshalb habe ich, um vergleichbare Mafse bieten zu können, aus dem mittleren Durchmesser des Metacarpus den ellipsenförmig gedachten Umfang berechnet und dann festgestellt, wieviel Prozent dieser vom Röhrbeinumfang über der Haut beträgt (Spalte 2 der Tabelle V). Dieser Prozentsatz beträgt im Durchschnitt bei den Laufpferden 52,96\%, bei den Schrittpferden $50,88 \%$, ist also bei den letzteren um ca. $2 \%$ geringer.

Ein Beispiel wird die Grösse dieses Unterschiedes deutlich machen. Denken wir uns 2 Pferde, ein Laufpferd und ein Schrittpferd, bei welchen das erwälnte Durchschnittsverhältnis zwischen Knochen- und Röhrbeinumfang besteht, also beim Laufpferd rund $53 \%$, beim Schrittpferd rund $51 \%$. Beide Pferde sollen gleiche Knochenstärke liaben. Dann müsste natürlich der Röhrbeinumfang über der Haut beim Schrittpferd ein grösserer sein als beim Laufpferd, und zwar würde er, wenn er beim Laufpferd $20 \mathrm{~cm}$ beträgt, beim Schrittpferd $\frac{53.20}{51}=20,8 \mathrm{~cm}$, also fast $21 \mathrm{~cm}$ gross sein müssen. Oder umgekehrt: Es ist anzunehmen, dass ein Laufpferd von $20 \mathrm{~cm}$ Röhrbeinumfang ebenso starke Mittelfussknochen hat, wie ein Schrittpferd von fast $21 \mathrm{~cm}$ Röhrbeinumfang. Diese Differenz ist nach meiner Meinung mitbedingt durch die kräftigere Ausbildung der Griffelbeine, etwas wohl auch durch den durchschnittlich stärkeren Behang. Ob die Sehnen und die Haut voluminöser entwickelt sind als bei den Laufpferden, habe ich nicht festgestellt. Bedeutender als der Unterschied zwischen beiden Rassen sind die Schwankungen innerhalb derselben, wie die Zahlen in Spalte 4 der Tabelle deutlich zeigen.

Um eine bessere Beurteilung dieser Verhältnisse zu ermöglichen, habe ich die Pferde nach dem besprochenen Prozentsatz geordnet und dann die 
Pferde jeder Rasse in fünf Gruppen geteilt. Die mittlere Gruppe C umfasst diejenigen Pferde, bei denen die Prozentsätze den Durchschnittszahlen der betreffenden Rasse am nächsten kommen, also bei den Laufpferden von $52-54 \%$, bei den Schrittpferden von $50-52 \%$. Die Pferde in Gruppe B haben einen um $2 \%$ geringeren Knochenumfang, die in Gruppe D einen um ebensoviel grösseren. Die äussersten Gruppen A und E enthalten die Extreme, die ein um fernere $2 \%$ günstigeres oder ungünstigeres Verhältnis zeigen; Gruppe A der Laufpferde ist sogar um $3 \%$ ungünstiger. Spalte 5 gibt den Durchschnittsprozentsatz der Gruppe an, Spalte 6 die Anzahl und Spalte 7 den Prozentsatz der Pferde, welche das Verhältnis zwischen Knochen- und Mittelfussumfang der betreffenden Gruppe haben.

Wir wollen nun annehmen, dass sämtliche Pferde innerhalb jeder der beiden Rassen einen gleichen Umfang des Mittelfusses (über der Haut gemessen) hätten. Daraus wäre aber nicht zu schliessen, dass nun alle Pferde auch gleichstarke Röhrbeinknochen hätten. Die Stärke derselben würde vielmehr mit den Zahlen der Spalte 4 resp. 5 zunehmen, nämlich in dem Verhältnis, wie dasjenige des Knochenumfanges zum Mittelfussumfange wächst. In der Gruppe C wäre die Knochenstärke eine mittlere. Darum wollen wir dieser Gruppe in Spalte 8 die Durchschnittsmafse der Knochenquerschnitte (806 resp. $1047 \mathrm{qmm}$ ) geben. Den Pferden der übrigen Gruppen wäre dann den Zahlen der Spalten 4 und 5 entsprechend ein kleinerer oder grösserer Knochenquerschnitt zuzusprechen, wie ihn die übrigen Zahlen der Spalte 8 aufweisen. Da im allgemeinen einem stärkeren Knochen auch eine grössere Tragkraft innewohnt als einem mit kleinerem Querschnitte, so würden die Knochen der letzten Gruppen für schwerere, die der ersten Gruppen für leichtere Pferde passend sein. Entspräche z. B. der Knochenquerschnitt der Gruppe $\mathrm{C}$ der Laufpferde einem Pferde von 10 Ztr., so würde sich der Knochen der Gruppe B mit seinem kleineren Querschnitt nur für ein Pferd von 9,3 Ztr. eignen, der der Gruppe A sogar nur für ein Pferd von 8,2 Ztr. ausreichen. Umgekehrt würden die Knochen der Gruppe D und E für Pferde von 10,7 und 11,6 Ztr. genügen. Diese Schwankungen in der Knochenstärke würden also vorhanden sein, trotz des gleichen Umfanges des Mittelfusses. Bei den Schrittpferden habe ich in Gruppe C, also als mittleres Gewicht, ein Pferd von 14 Ztr. angenommen. Die Zahlen in den übrigen Gruppen der Schrittpferde zeigen dasselbe Gewichtsverhältnis wie in den Laufpferdegruppen.

Um vorstehende Resultate zu gewinnen, sind wir von Pferden mit gleichem Mittelfussumfang ausgegangen. Nun kann man aber auch umgekehrt lauter Pferde mit gleicher Knochenstärke annehmen und daraus auf den verschiedenen Mittelfussumfang in den 5 Gruppen schliessen. Hätten wir z. B. 100 Laufpferde, alle von einer Knochenstärke, die einem Mittelfuss von $21 \mathrm{~cm}$ Umfang zukommt, so würden nur die 46 Pferde der Gruppe C diesen Umfang wirklich aufweisen, während die Pferde der Gruppe B 21,8 cm, die der Gruppe A sogar 23,0 cm Röhrbeinumfang zeigen würden. Dagegen hätten die Pferde der Gruppen $\mathrm{D}$ und $\mathrm{E}$ bei 
derselben Stärke des Röhrbeinknochens nur 20,2 resp. 19,5 cm Mittelfussumfang.

Bei den Belgiern habe ich einen Umfang von $23 \mathrm{~cm}$ als den normalen angenommen. Unter 100 Belgiern von gewünschter Knochenstärke wären dann nur die 22 der Gruppe $C$ mit dem normalen Röhrbeinumfang von $23 \mathrm{~cm}$; die 22 der Gruppe B würden 23,9 cm, die 14 der Gruppe A 26,1 cm haben, während die 28 Pferde der Gruppe D nur 22,1, die 14 der Gruppe E sogar nur 21,3 cm aufzuweisen brauchten, um die normale Grösse des Knochenquerschnittes zu haben.

Hält man die durch meine Untersuchungen gewonnenen Zahlen für fähig, verallgemeinert $\mathrm{zu}$ werden, und will man aus den vorstehenden Erwägungen auf den Wert der Röhrbeinmessungen am lebenden Pferde schliessen, so kommt man zu folgendem Resultat:

Unter 100 Laufpferden (Schrittpferden) entspricht bei 46 (22) der Umfang des Metacarpus dem durch Messung des Mittelfusses ermittelten Malse. Bei 16 (22) Pferden (Gruppe B) hat der Knochen eine um 7,5\% $(8,3 \%$ ) geringere, bei weiteren 10 (14) Pferden (Gruppe A) eine um $17,5 \%(23 \%)$ geringere Tragkraft, als das Röhrbeinmals vermuten lässt. Dagegen haben 16 (28) Pferde (Gruppe D) eine um 7,5\% (7,5\%) grössere und fernere 12 (14) Pferde (Gruppe E) sogar eine um 16\% (15\%) grössere Tragkraft, als dem Röhrbeinumfange entspricht.

Diese Abweichungen erscheinen erheblich durch die geringe Zahl der Pferde mit einem normalen Verhältnis zwischen Knochen- und Mittelfussumfang, besonders der Belgier $(22 \%)$. Betrachtet man aber die Grösse der Schwankungen, so erscheinen die Abweichungen viel unbedeutender. Am klarsten zeigen das die Gewichtszahlen in Spalte 9. Schwankungen von kaum einem Zentner, wie sie Gruppe B und D zeigen, können schon durch verschiedenen Futterzustand verursacht werden, ohne dass dieser ein extremer zu sein brauchte. Wir könnten daher diese beiden Gruppen immer noch als normale bezeichnen. Demnach blieben nur die Pferde der äussersten Gruppen A und E, bei denen die Knochenstärke eine anormale ist. Die grössere Knochenstärke in Gruppe E wird man sich gern gefallen lassen. Nur die Pferde der Gruppe A hätten eine Knochenstärke, die man als eine relativ mangelhafte bezeichnen müsste. Das wären $10 \%$ der Laufpferde und $14 \%$ der Schrittpferde. - Ich meine aber, wenn eine Regel der Lehre vom Exterieur uns in 86 oder gar $90 \%$ aller Fälle den richtigen Schluss auf die Leistungsfähigkeit eines Organes gestattet, so können wir damit zufrieden sein.

Ein Vergleich von Lauf- und Schrittpferden auf Grund der Zahlen in Tabelle V lässt das Verhältnis von Knochen- und Mittelfussumfang bei den Laufpferden konstanter erscheinen, weniger durch die Grösse der Abweichungen als durch die Zahl der Pferde (in der Mittelgruppe) mit normalem Verhältnis.

Das kommt daher, dass die untersuchten Laufpferde alle einer bestimmten Form angehörten, während unter den Schrittpferden ausser den Belgiern auch englische und dänische Formen vertreten waren. 
Zur besseren Veranschaulichung stellten wir der Knochenstärke ein entsprechendes Körpergewicht des Pferdes gegenüber. Das würde nur dann gerechtfertigt sein, wenn die Beanspruchung der Knochen immer dem Körpergewicht proportional wäre. Über diesen Punkt sind die Autoren aber sehr verschiedener Ansicht. Einen ganz abweichenden Standpunkt nimmt z. B. RAuber ein. Er sagt: ${ }^{1}$ ) „Die typische Belastung der Röhrenknochen ist die Spannung ihrer Nuskulatur. Das Extremitätenskelett lässt sich auffassen als eine Gruppe von Gegenresultierenden, deren Komponenten durch antagonistische Muskelkräfte gegeben sind." Zschоcke hält es für höchst wahrscheinlich, dass bei einzelnen Knochen, z. B. am Hüftgelenk der Pferde, der Muskeldruck wesentlich grösser ist als der Belastungsdruck, und er schreibt deshalb einen Teil der Knochenform und auch der innern Architektur der Muskelwirkung zu. ${ }^{2}$ ) Während Meyer und WolfF „in der normalen, aufrechten Stellung diejenige Anforderung an die Knochen erblicken, welche für deren Aufbau massgebend war", erscheint es ZscноскE unrichtig, die Struktur des Knochens (in Hinsicht auf die Maximalleistung desselben) "lediglich nach der Inanspruchnahme beim blossen Stehen zu beurteilen". Thm scheint es vielmehr, dass die Knochen bei der Bewegung, z. B. beim Auf- und Niedersprung, am meisten geprüft und mitgenommen werden. - Das wird man ohne weiteres zugeben. Aber wie soll man diese Inanspruchnahme zahlenmässig ausdrücken? Ich musste es in meiner Zusammenstellung in Tabelle $\mathrm{V}$ schon beim Körpergewicht bewenden lassen.

Eine andere Frage ist es ja, ob man in Erwägung der bei Laufpferden grösseren Inanspruchnahme der Knochen durch Sprung und Lauf für die Laufpferde nicht stärkere Knochen fordern sollte, als diesen ihrem Körpergewicht nach gegenüber den Schrittpferden zukommt.

Ich schliesse hieran gleich die Betrachtung der Wandstärke der Knochen, obgleich ich diese Mafse erst nach Ausführung der Biegeversuche gewinnen konnte. Ich habe mit einem sehr genau gearbeiteten Tastzirkel gemessen; doch wurde hin und wieder wegen der Zersplitterung des Knochens das Resultat ungenau oder war auch gar nicht festzustellen.

Die Stärke der Wände wurde an 4 Stellen gemessen, welche in Fig. 8 und 9 durch punktierte Linien bezeichnet sind, nämlich in der Mitte der volaren Fläche (v), dieser gegenüber an der dorsalen Wandung (d), ferner an der lateralen Seite (l) und dann an der stärksten Stelle der medialen Wand $(m)$. Nachdem die absolute Stärke der Wände festgestellt war, wurde daraus berechnet, wieviel Prozent der Durchmesser jeder Wand vom mittleren Radius des Röhrbeins betrug. Danach wurde noch die absolute und relative Wandstärke jedes Knochens festgestellt. Tabelle VI zeigt die gewonnenen Resultate.

Als wichtigstes Ergebnis geht aus diesen Zahlen hervor, dass die Wandstärke bei den Schrittpferden absolut zwar grösser, relativ aber kleiner

1) A. A. RAuber, Elastizität und Festigkeit der Knochen, Leipzig 1876, Seite 73.

2) Zsсноске, Untersuchungen über das Verhältnis der Knochenbildung, Zürich 1892, Seite 17. 
ist als die der Laufpferde. Die mittlere Wandstärke beträgt im Durchschnitt $9,07 \mathrm{~mm}$ bei Schrittpferden, bei Laufpferden $8,71 \mathrm{~mm}$, aber bei

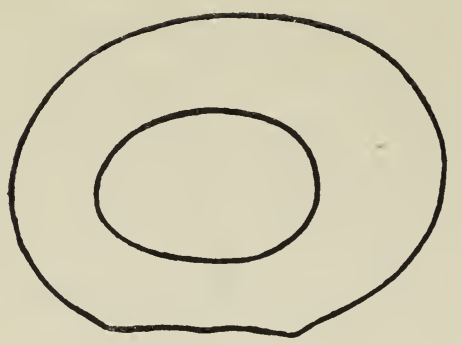

Fig. 2. $\mathrm{S} 251$.

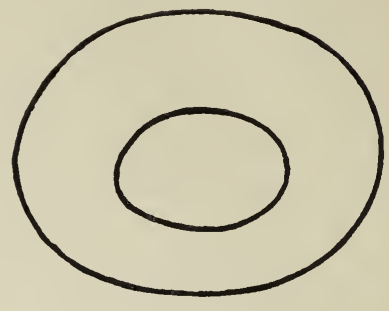

Fig. 3. L $46 \mathrm{r}$.

Fig. 2 und 3. Beide kommen in ihrer mittleren Wandstärke dem Durchschnitt ihrer Rassen am nächsten.

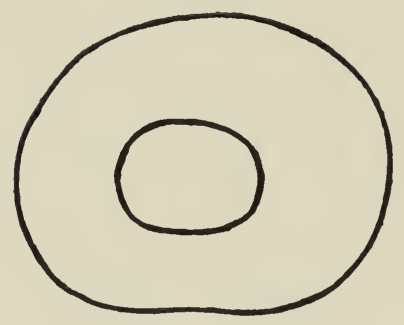

Fig. 4. L $71{ }^{1}$ )

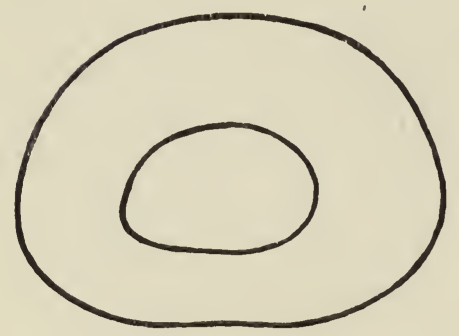

Fig. 6. S2l.

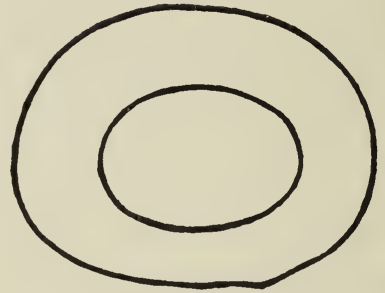

Fig. 5. L 44 r.

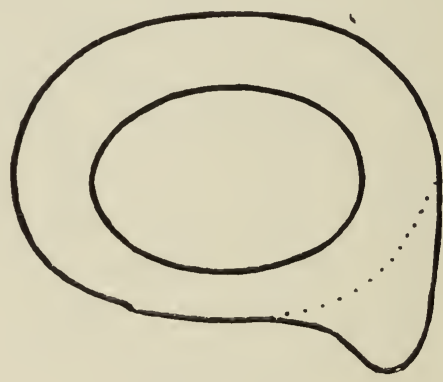

Fig. 7. $\mathrm{S} 6 \mathrm{r}$.

Fig. 4-7. Extreme in den Wandstärken bei Laufpferden (L 7 l. und L 44 r.) und bei Schrittpferden (S 2 1. und S6r.).

Schrittpferden $49,1 \%$, bei Laufpferden $53,9 \%$ des mittleren Radius (vergl. Fig. 2 und 3 ).

1) Sämtliche Figuren der Knochenquerschnitte sind so orientiert, dass sich immer ein rechter und ein linker Metacarpus gegenüberstehen, so dass die Lage der Seiten in den Figuren auch jedesmal der natürlichen Lage der Knochenwände entspricht. Eine Ausnahme hiervon machen nur die letzten drei Querschnitte (Fig. 21-23), welche alle drei rechtsseitige Knochen darstellen. 
Die Schwankungsgrenzen sind:

\begin{tabular}{|c|c|c|c|c|}
\hline & \multicolumn{2}{|c|}{ Absolut $m m$} & \multicolumn{2}{|c|}{ Relativ $\%$ des mittleren Radius } \\
\hline & minimal & maximal & minimal & maximal \\
\hline $\begin{array}{l}\text { Bei Laufpferden . } \\
\text { Schrittpferden }\end{array}$ & $\begin{array}{l}7,4 \text { (No. L } 42 \text { r.) } \\
7,4 \text { (No. S } 6 \text { l.) }\end{array}$ & $\begin{array}{l}11,1 \text { (No. L } 17 \text { r.) } \\
10,6 \text { (No. S } 2 \text { l.) }\end{array}$ & $\begin{array}{l}45 \% \text { (No. L } 44 \text { r. } \\
36 \% \text { (No. S } 6 \text { r.) }\end{array}$ & $\begin{array}{l}\left.63 \% \text { (No. L } 71 .)^{1}\right) \\
56 \% \text { No. S } 21 .)\end{array}$ \\
\hline
\end{tabular}

(Vergl. Fig. 4 bis 7.)

Als ich die Knochen des einzigen von mir untersuchten Hengstes (L 1) so starkwandig fand, dass sich dazu nur ein Gegenstück (L 7) finden liess, hielt ich es für möglich, dass diese Starkwandigkeit der Knochen eine Eigenart des männlichen Geschlechtes sei, die sich, wenn auch abgeschwächt, auch bei den Wallachen zeigen würde. Ich berechnete deshalb die durchschnittliche Wandstärke der Wallachenknochen für sich allein und verglich sie mit den Durchschnittszahlen der Tabelle VI. Die nachstehende Zusammenstellung zeigt das Ergebnis.

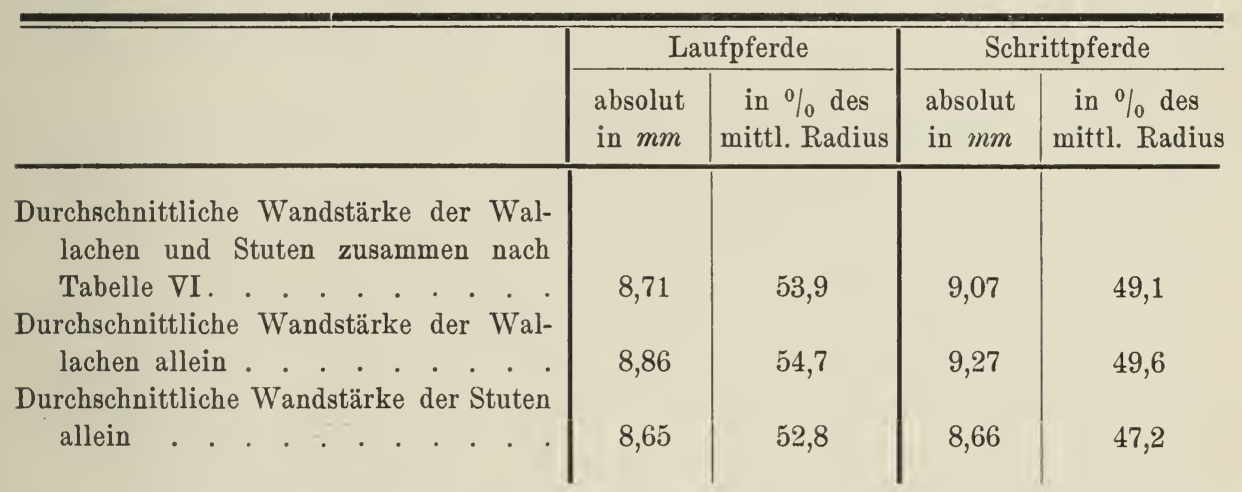

Es sind demnach die Knochen der Wallache etwas starkwandiger als die der Stuten, in beiden Rassen. Diese Differenz ist im Vergleich zu den grossen Schwankungen innerhalb beider Rassen (um 16 resp. 20\%) sehr gering. Einige der Wallache zeigen auch eine Wandstärke, die bedeutend hinter der durchschnittlichen der betr. Rasse zurücksteht.

Ausserdem fnden sich bei den Laufpferden sowohl als bei den Schrittpferden Stuten, welche den Wallachen durchaus gleichkommen in der Wandstärke. Ist doch auch das Pferd, welches die gleiche Wandstärke wie der Hengst zeigt, eine Stute (L 6) und kein Wallach. Die geringe Knochenwandstärke einiger Wallache lässt sich vielleicht auf eine sehr frühzeitige Kastrierung zurïckführen.

Da sich unter den untersuchten Schrittpferden weit mehr Wallache $(58 \%$ ) befinden als unter den 59 Laufpferden (inkl. des Hengstes nur

1) L 1 1. zeigt $64 \%$. Doch habe ich diese Zahl hier nicht angeführt, weil L 1 der einzige Hengst unter den untersuchten Pferden ist. 
$19 \%$ ), so müssen auch die Zahlen für die Wandstärke der Knochen bei den Schrittpferden durch die grössere Wandstärke der Wallachenknochen mehr beeinflusst sein als die der Laufpferde. Wäre der Prozentsatz an Wallachen bei den Schrittpferden ebenso gering wie bei den Laufpferden, so würden die allgemeinen Durchschnittszahlen der Tabelle VI S, sowohl die absoluten wie die relativen, etwas kleiner sein; doch wäre diese Änderung so geringfügig, dass dadurch das Verhältnis zwischen der Wandstärke der Lauf- und Schrittpferdeknochen nicht wesentlich geändert würde.

Die Verteilung der Knochenmasse um die Markhöhle herum wird durch folgende Durchschnittszahlen erläutert.

\begin{tabular}{|c|c|c|c|c|c|c|c|c|c|c|}
\hline \multirow[b]{2}{*}{$\begin{array}{c}\text { Stärke der } \\
\text { Knochenwand }\end{array}$} & \multicolumn{5}{|c|}{ Laufpferde } & \multicolumn{5}{|c|}{ Schrittpferde } \\
\hline & 咅 & 苞 & 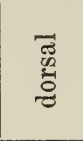 & : & 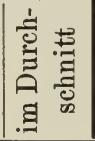 & $\frac{\bar{m}}{\circ}$ & 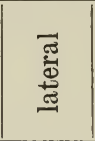 & 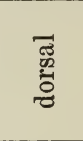 & 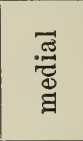 & 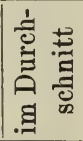 \\
\hline $\begin{array}{l}\text { a) absolut in } m m \\
\text { b) in } \% \text { des mittleren }\end{array}$ & 5,99 & 8,63 & 9,25 & 10,98 & 8,71 & 6,29 & 9,11 & 8,99 & 11,89 & 9,07 \\
\hline Radius . . . . . & 37,0 & 53,4 & 57,3 & 68,0 & 53,9 & 34,2 & 49,4 & 48,3 & 64,3 & 49,1 \\
\hline
\end{tabular}

Diese Verteilung der Knochenmasse, also das Bild des Querschnittringes ist aber individuell so verschieden, dass es schwer fällt, zwei darin

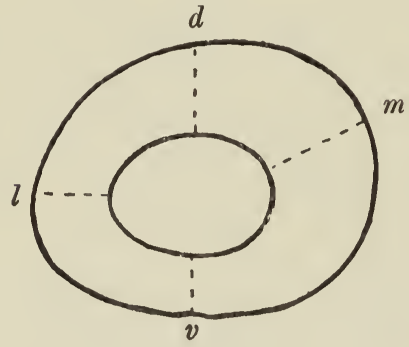

Fig. 8. L 331 .

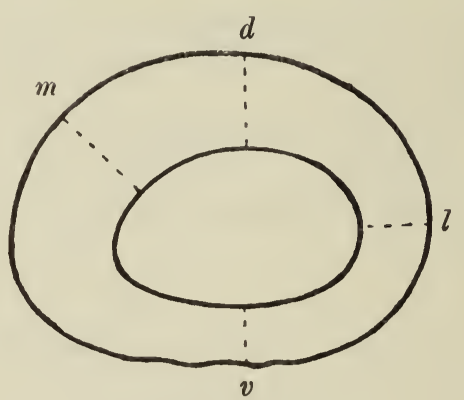

Fig. 9. S $10 \mathrm{r}$.

Fig. 8 und 9 zeigen schön das Stärkerwerden der Wände von der volaren über die laterale und dorsale zur medialen Seite hin. Die punktierten Linien geben die Stellen an, an welchen die Wandstärke gemessen wurde.

übereinstimmende Knochenpaare zu finden, selbst wenn man nicht auf die absoluten Mafse, sondern nur auf den prozentualen Anteil der Wände am mittleren Querschnittradius sieht. Als Regel lässt sich aber auf Grund vorstehender Zahlen folgendes aufstellen (vergl. Fig. 8 und 9). Am schwächsten ist die volare, am stärksten die mediale Wand, und zwar nach vorn zu. Bei Laufpferden ist in den meisten Fällen die vordere dorsale Wand stärker als die laterale, während bei Schrittpferden dies Verhältnis meist umgekehrt ist. Im allgemeinen kann man aber sagen, dass die Wandstärke von der volaren Fläche aus über die laterale und dorsale fortschreitend nach der medialen Seite hin zunimmt. Von den vorigen 
Figuren zeigen No. L 44, S 6 und S 2 diese Zunahme der Wandstärke. Besonders deutlich aber ist das ausgeprägt bei No. S 10 r. und L $33 \mathrm{l}$. (Fig. 8 und 9). - Sehr bezeichnend für die Variabilität der Wandstärke (Fig. 7 und 8) ist es, dass unter den 200 Knochen nicht einer zu finden ist, welcher die als Durchschnitt berechneten Mafse hat, selbst nicht die prozentualen. Dem Durchschnitt der Preussen kommen am nächsten L 46 r. (Fig. 3) und L 58 l., dem der Belgier S 16 l., S 25 l. (Fig. 2) und S $9 \mathrm{r}$.

Diese Mafsverhältnisse stimmen überein mit den Ausführungen ErcHBaUms. ${ }^{1}$ ) Nach diesem lässt sich die starke Ausbildung der medialen Wand gegenüber der der lateralen aller Knochen der Extremitäten leicht durch den Umstand erklären, „dass infolge der Lage der Schwerlinie des Körpers und der entweder medialwärts gelegenen Gelenkhervorragungen bezw. Vertiefungen oder der höher oder tiefer gestellten medialen Abteilungen der letzteren und der hiermit verbundenen stärkeren Belastung die Mittelkraft medial von der Knochenachse die Querschnitte des Knochens schneiden, die neutrale Achse also lateralwärts von derselben und damit weiter entfernt von der Innenwand gelegen sein muss. Die letztere muss daher in grösserem Mafse auf Druck beansprucht werden, wie die Aussenwand auf Zug.".

Dass die vordere, dorsale Wand stärker als die hintere, volare ausgebildet ist, hat nach demselben Verfasser seinen Grund in der leichten Krümmung des Metacarpus, dessen konkave Seite nach vorn und dessen konvexe Seite nach hinten gerichtet ist. Diese Krümmung soll den Zweck haben, die Gewalt des Stosses zu mildern, was wegen der fast senkrechten Stellung dieses Knochens nötig ist. Durch diese Krümmung aber wird die dorsale Wand der neutralen Achse des Knochens genähert, die volare Wand dagegen entfernt, woraus sich eine grössere Beanspruchung und daher stärkere Ausbildung der Vorderwand erklärt. Da also laterale und dorsale Wand die stärksten sind, ist es natürlich, dass an der Berührungsstelle beider die Kompakta die grösste Dicke zeigen muss.

Auffallend ist es aber, dass die dorsale Wand bei den Schrittpferden im Durchschnitt schwächer ist als die laterale, während bei den Laufpferden die Verhältnisse gerade umgekehrt liegen. Das würde erklärlich sein, wenn die Biegung des Metacarpus der Schrittpferde eine geringere wäre als bei den Laufpferden. Leider habe ich bei den Knochenmessungen die Krümmung nicht beachtet, und nach dem Zerbrechen der Knochen lassen sich diese Verhältnisse nicht mehr feststellen. Doch könnte für die geringere Stärke der dorsalen Knochenwand bei den Schrittpferden auch der grössere Querschnitt mitbestimmend sein.

Wahrscheinlich hat Eichвaum bei seinen Untersuchungen nur das Laufperd beachtet und wohl nur an wenigen Exemplaren. Die für die Stärke der Kompakta des Metacarpus von ihm (Seite 99) angegebenen Mafse $(8,5 \mathrm{~mm}$ als grösste Stärke der vorderen WVand und $7,5 \mathrm{~mm}$ als stärkste hintere) sind nach meinen Messungen nicht als normale anzusehen.

$\left.{ }^{1}\right)$ Dr. FR. Eichваum, Beiträge zur Statik und Mechanik des Pferdeskeletts, Berlin 1890. 
Nach den in Tabelle VI gegebenen Zahlen ist die Durchschnittsstärke der dorsalen Wand in der Mitte des Knochens bei Laufpferden 9,24 mm, müsste also, da nach Еichваum die Wand nach oben noch stärker wird, mindestens $9,5 \mathrm{~mm}$ betragen. Demgegenüber ist die volare Wand mit 7,5 $\mathrm{mm}$ Dicke als auffallend stark zu bezeichnen.

Ich glaube, dass die individuelle Verschiedenheit in der Stärke der Knochenwandungen viel grösser ist, als allgemein angenommen wird. Auch hier sieht man wieder, wie wichtig grosses Untersuchungsmaterial ist.

Von der Variabilität der Querschnittformen geben die Fig. 10-19 ein Bild. Doch muss ich hierzu bemerken, dass die Figuren wegen der Zersplitterung der Knochen teilweise rekonstruiert werden mussten. Deswegen liessen sich auch keine photographischen Bilder herstellen. Dass auffallende Verschiedenheiten anch zwischen den beiden Knochen eines Paares vorkommen können, zeigen L 41 links und rechts.

Bei der Betrachtung dieser Variabilität drängt sich der Gedanke auf, dass vielleicht die Verschiedenheit in der Verteilung der Wandstärke mit durch eine Verschiedenheit der Belastung des Röhrbeins bei der Arbeit des Pferdes verursacht sein könnte. Dr. Hirsch hat wenigstens nachgewiesen, dass beim Nenschen die Wände der Tibia ganz verschieden ausgebildet sind, je nachdem der Beruf eine spezifische Inanspruchnahme des Unterschenkels erfordert. ${ }^{1}$ ) Bei den Pferden wäre hierbei nicht nur die Art der Arbeit, sondern auch die Stellung des Fesselgelenks (flach oder steil, zeheneng oder zehenweit) von Einfluss. Auch wird bei breiten Pferden im schweren Zug, bei welchem sie den Huf genau in die Mittellinie stellen, so dass das Bein schräg nach innen zu stehen kommt, die Belastung der lateralen Röhrbeinwand eine relativ stärkere sein als bei schmalen Pferden, bei welchen die Schulterblätter doch näher beieinander liegen. Vielleicht ist das auch der Grund dafür, dass bei den Schrittpferden im grossen Mittel die laterale Wand etwas stärker ist als die dorsale, während es bei den Laufpferden gerade umgekehrt ist.

Weiter konnte ich diesen Gedanken an meinem Material nicht verfolgen. Dazu wäre nötig gewesen, dass ich die Art der Arbeit der Pferde genau gekannt hätte. An die Röhrbeine der Pferde L 7 und L 49, beide von den 1. Gardedragonern, Betrachtungen über die Wandstärke bei Reitpferden zu knüpfen, erschien mir nicht angängig. Dazu wäre eine grössere Zahl von Objekten nötig gewesen. Dasselbe gilt von dem einen Artilleriepferd L 12 und dem einen Zirkuspferd L 1, das überdies noch als einziger Hengst den übrigen Pferden doch nur sehr bedingt gegenübergestellt werden durfte.

Die Betrachtung der Knochenwände lenkt unsere Aufmerksamkeit auf die Beschaffenheit der Markhöhle, der Spongiosa und des Markes. Hierauf

1) Diese Mitteilung verdanke ich der Freundlichkeit des Herrn Prof. Dr. RUdeLoff, unter dessen Leitung Herr Dr. Hirsch seine Untersuchungen im Königl. Material-Prüfungsamt zu Gross-Lichterfelde ausführte. Die darauf bezügliche Veröffentlichung habe ich trotz aller Mühe nicht finden können. 


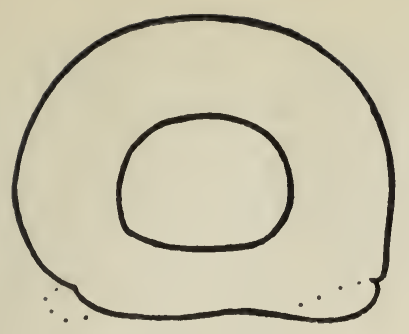

Fig. 10. L 181 .

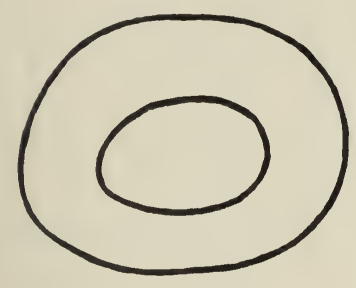

Fig. 12. L 421 .

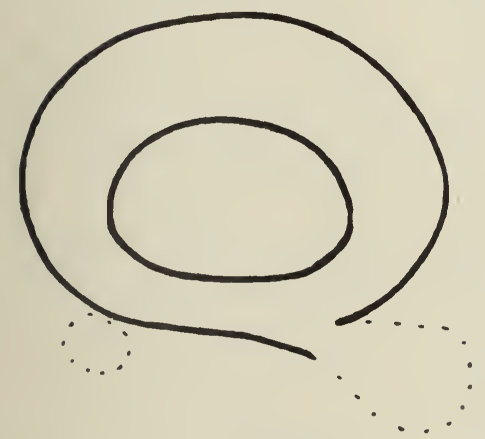

Fig. 14. S 361.

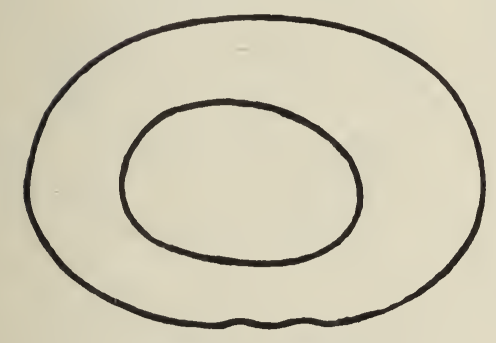

Fig. 16. S 241.

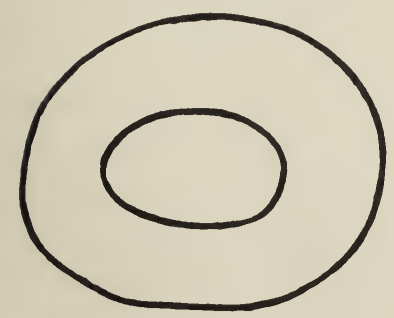

Fig. 18. L 41 l.

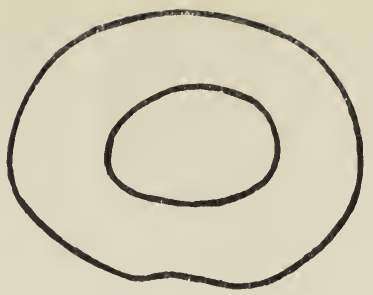

Fig. 11. L $40 \mathrm{r}$.

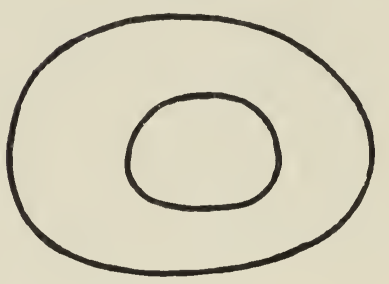

Fig. 13. L $15 \mathrm{r}$.

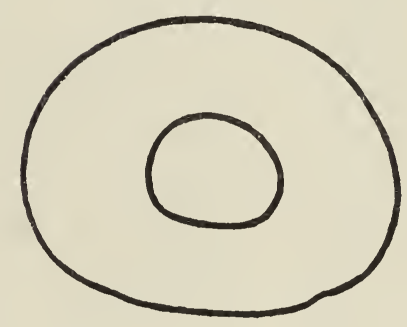

Fig. 15. L $17 \mathrm{r}$.

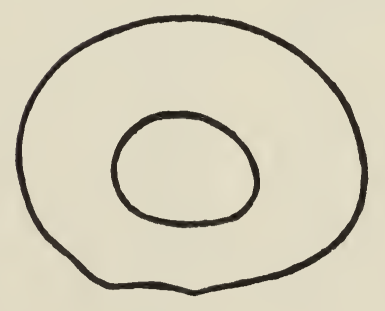

Fig. 17. L $30 \mathrm{r}$.

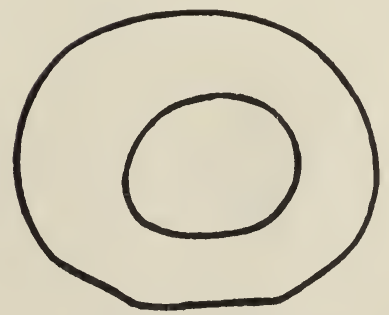

Fig. 19. L $41 \mathrm{r}$.

Fig. 10-19, Verschiedene Formen der Knochenringe, d. h. der Verteilung der Knochenmasse um die Markhöhle. 
habe ich leider bei dem Zerbrechen der Knochen zu wenig geachtet. Auffallend war mir nur die sehr grosse Verschiedenheit in der Farbe des Markes, welche vom blassen Weissgrau bis zum deutlichen Rot variierte. Ausserdem schien mir die Spongiosa sehr verschieden stark ausgebildet. Doch habe ich über beide Punkte nichts notiert, so dass ich daran keine Betrachtungen schliessen kann. Betreffend die verschieden starke Ausbildung der Spongiosa finde ich bei Zschocke nur die Notiz, dass die Markhöhle bei älteren Tieren „oft eigenmächtig und rücksichtslos in die Epiphysen vorzudringen scheine, so dass die Spongiosa merkwürdig reduziert wird". Dieser Verlust an Knochensubstanz müsste auf das spez. Gewicht der ganzen Knochen einwirken, und zwar derart, dass die Knochen älterer Tiere

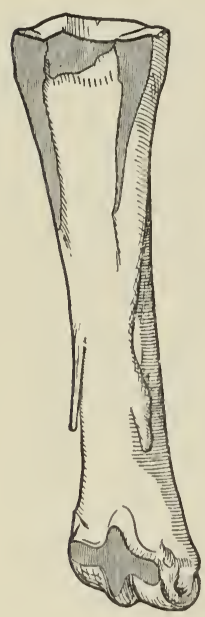

Fig. 20. L 43 l.

1/4 nat. Gr. Starke Verwachsung schwacher Griffelbeine mit dem Metacarpus. Die senkrecht schrafflerten Flächen sind die für die Biegeversuche abgefrästen Auflagestellen. spezifisch etwas leichter sein müssten als die jüngerer Tiere. Doch ist eine darauf basierendeParallele von Alter und spez. Gewicht bei den untersuchten Knochen (Tabelle XIII) nicht zu erkennen. Die Differenz an Knochensubstanz wird so gering sein, dass sie durch anderes verdeckt wird. Zschocke hält übrigens die Annahme für prüfenswert, dass dieses Schwinden der Spongiosa eine Folge der Domestikation sei, z. B. bei Rindern, die ständig im Stalle gehalten werden.

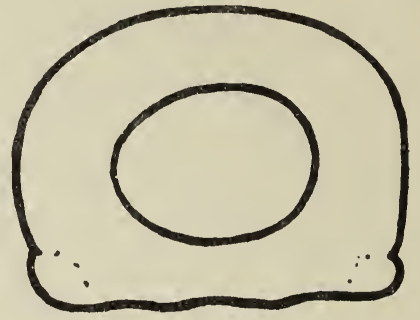

Fig. 21. S 31 r.

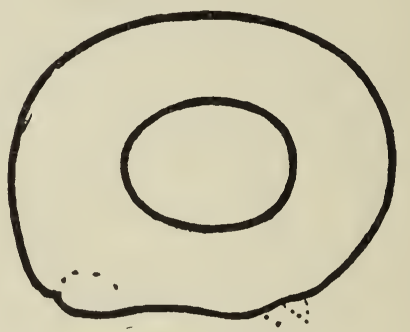

Fig. 22. L 54 r.

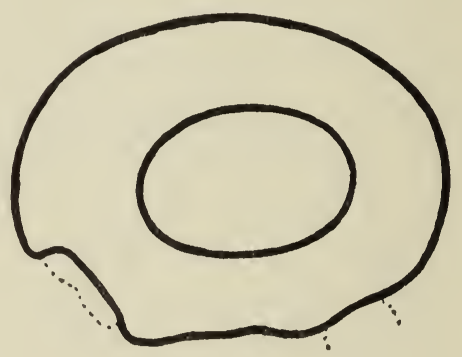

Fig. 23. S 35 r.

\section{Die Griffelbeine.}

Von diesen habe ich leider nur $1 \mathrm{Ma}$ s nehmen

Fig. 21-23. 1/1. Veränderungen der Querschnittformen durch Verwachsung der Griffelbeine mit dem Röhrbein. Die Punkte deuten die Lage der Griffelbeine an.

können, nämlich die Länge. Diese ist aber weniger charakteristisch als ihre Stärke und der Grad der Verwachsung. Letzteres lässt sich leider nicht durch Zahlen ausdrücken. Die Stärke wieder konnte wegen der Verwachsung nicht gemessen werden. Nun wäre es ja in vielen Fällen möglich gewesen, nach der Ausführung der Biegeversuche die Griffelbeine vom Röhrbein mit Gewalt zu trennen und dann das Volumen festzustellen. Aber zur Ermöglichung der Biegeversuche war von allen Röhrbeinen ein grosses 
Stück weggeschliffen worden (siehe Fig. 20), so dass die gewinnbaren Volumzahlen wertlos sein mussten. Ich habe darum diese Arbeit nicht

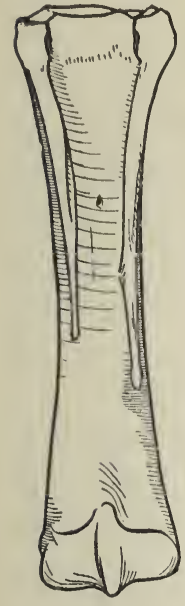

$A$

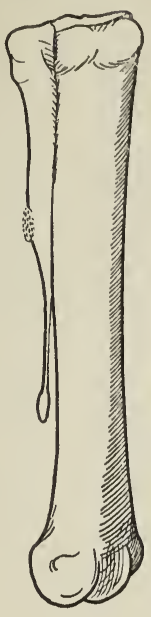

$B$

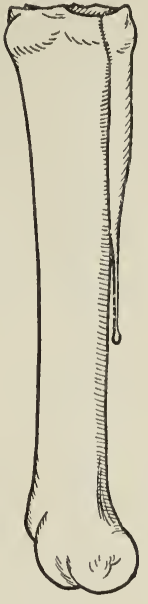

$C$
Fig. 24. $1 / 4$ nat. Gr. Röhrbein No. L 26 l. $A$ von der hinteren, $B$ von der inneren, $C$ von der äusseren Seite gesehen, mit auffallend schwacher Ausbildung der Griffelbeine. ausführen können und war ganz auf mein freies Urteil angewiesen. Hiernach glaube ich folgendes behaupten zu dürfen:

Die beiden Griffelbeine eines Röhrbeines sind selten ganz übereinstimmend. Bei grösserer Differenz war das innere immer das stärkere. Metacarpus IV ist also in der Rückbildung weiter vorgeschritten. Im allgemeinen waren die Griffelbeine der Schrittpferde voluminöser; die der Laufpferde verjüngten sich nach unten mehr. Alle Griffelbeine, auch die dünnsten, zeigten unten ein kleines Gelenkköpfchen. Die Stärke der Griffelbeine ging nicht parallel mit dem Grade der Verwachsung.

Letztere war ganz individuell. Sie beeinflusste oft das Bild des Röhrbeinquerschnittes. Gewöhnlich erschien die Wand des Röhrbeins in der Nähe der Verwachsungsstelle aufgetrieben. Das war selbst dann öfter der Fall, wenn das Griffelbein am Röhrbein nur fest anlag, ohne mit ihm verwachsen zu sein. Fig. 20, das Röhrbein L 43 l. darstellend, zeigt solche tief verwachsenen Griffelbeine, die an sich von geringer Stärke sind.

Wie das Bild des Querschnittes durch die Verwachsung des Griffelbeines beeinflusst werden kann, ist aus den Fig. 21-23 (S 31 r., L 54 r. und $\mathrm{S} 35 \mathrm{r}$.) $\mathrm{zu}$ erkennen, auch aus Fig. 7, 10 u. 14.

Eine extreme Ausbil-

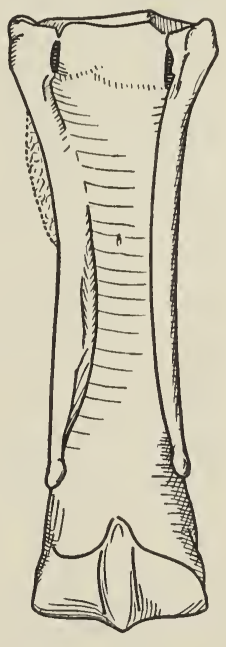

$A$

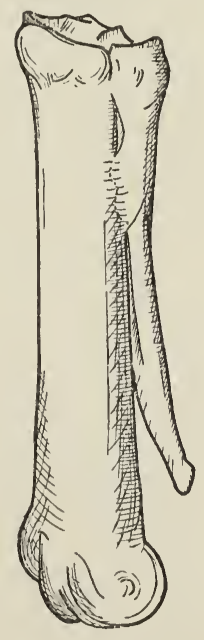

$B$

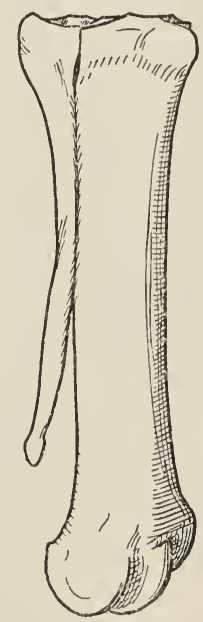

$C$

Fig. 25. $1 / 4$ nat. Gr. Röhrbein No. S 23 r. $A$ von der hinteren,

$B$ von der inneren, $C$ von der äusseren Seite gesehen, mit extremer Ausbildung beider Griffelbeine, besonders des inneren.

dung der Griffelbeine zeigen L 26 und S 23. Letzterem kann man noch No. D aus den angefügten Torsionsversuchen an die Seite stellen. Während die Rückbildung bei L 26 (Fig. 24), besonders des äusseren Griffelbeines, 
einen sehr hohen Grad erreicht hat, machen die Griffelbeine von S 23 (Fig. 25) fast den Eindruck, als hätten sie noch dazu beigetragen, die Körperlast zu tragen. Bei No. D war das innere Griffelbein wohl noch voluminöser als bei S 23, aber durch seine starke Verwachsung erscheint es doch mehr als ein Appendix des Röhrbeins. S 23 dagegen zeigt eine so selbständige und dabei doch kräftige Ausbildung der beiden Griffelbeine, dass man versucht ist, es als ein Bindeglied zwischen Equus caballus und dem Pliohippus resp. dem Hipparion zu betrachten. Bei Laufpferden habe ich eine auch nur annähernd so starke Ausbildung der Griffelbeine nie gefunden, bei Schrittpferden wiederum keine so starke Rückbildung wie bei L 26 .

\section{Zusammenfassung der Messresultate an den Röhrbeinen.}

In der Tabelle VII habe ich sämtliche Messresultate noch einmal zusammengestellt. Daraus ergibt sich folgendes: Die absoluten Mafse der Schrittpferderöhren übertreffen sämtlich die der Röhrbeine der Laufpferde, und zwar ist das absolute Gewicht grösser um 30,7\%, das Volumen um $23,7 \%$, die Röhrenlänge um $1,3 \%$, der mittlere Durchmesser um $12,4 \%$, der Flächeninhalt des totalen Querschnittes um 29,9\% und die Wandstärke um $4 \%$. Dagegen ist das spez. Gewicht $1,2 \%$ geringer, ebenso der prozentuale Anteil der Wandstärke am mittleren Querschnittradius, nämlich um fast $10 \%$, was beides meiner Ansicht nach in kausalem Zusammenhang steht. Die grössere Gedrungenheit der Schrittpferderöhren kommt doppelt zum Ausdruck: einmal dadurch, dass das Röhrbein im Verhältnis zur Widerristhöhe um 2,6\% kürzer ist als bei den Laufpferden, und zweitens dadurch, dass der mittlere Durchmesser des Metacarpus im Verhältnis zu dessen Länge um $11 \%$ grösser ist. Ferner fand sich noch ein Unterschied darin, dass das Röhrbein der Schrittpferde flacher gebaut war als das der Laufpferde; die Differenz des prozentualen Anteiles des kleinen vom grossen Querschnittdurchmesser beträgt 5,8\%. Ausserdem ist endlich auffallend, dass bei den Schrittpferden die dorsale Knochenwand schwächer ist als die laterale, während es bei den Laufpferden umgekehrt ist. Ausserhalb des Querschnittzylinders fallen vom Volumen bei beiden Rassen gleiche Anteile der Masse des Metacarpus.

Die Tabelle gibt gleichzeitig die Schwankungsgrenzen. Diese zeigen deutlich, dass die Differenzen innerhalb beider Rassen weit grösser sind als zwischen denselben.

\section{Morphologische Asymmetrie der Röhrbeinpaare.}

MECkEL bezeichnet es in seinen Untersuchungen „über die seitliche Asymmetrie im tierischen Körper"1) als ein sehr grosses Unrecht, dass man dem Knochenskelett die Ehre angetan habe, es als das am meisten symmetrische hinzustellen. SchwarzNecker weist in seiner Pferdezucht ${ }^{2}$ ) auf die häufige Ungleichheit der Sprunggelenke hin. Er hält es für möglich,

1) Halle 1822.

2) Pferdezucht, 4. Auflage, S. 288. 
dass „der linke Fuss des Pferdes, ähnlich wie der rechte Arm des Menschen, durch Übung massiger sich entwickelt als der gegenseitige, und zwar deshalb, weil die Pferde im Naturzustande mit Vorliebe links galoppieren". Das müsste sich natürlich auch am Skelett zeigen. Wie Schwarznecker die linke, so hält BRoca die rechte Seite für die stärker entwickelte. Klarheit darüber aber konnten erst exakte Messungen bringen. Die Symmetrieverhältnisse am Pferdeskelett unterzog K. Heuss, dem ich auch obige Zitate Meckels und Brocas entnehme, eingehenden Untersuchungen. ${ }^{1}$ ) Er stellte an 24 Pferdeskeletten neben denen von 15 anderen Perissodaktylen die Knochenmafse fest, musste sich aber auf die Untersuchung von Länge und Umfang beschränken. Nur in wenigen Fällen konnte er auch das Gewicht feststellen. Das Längenmals des Metacarpus nahm er wie bei den vorliegenden Untersuchungen; der Umfang wurde aber „dicht über der Gelenkrolle am distalen Ende" gemessen, was bei meinen Untersuchungen in Hinsicht auf die Prüfung der Biegefestigkeit unzweckmässig gewesen wäre. Wegen dieser verschiedenen Messmethode lassen sich auch die von Heuss gewonnenen Zahlen mit den vorliegenden nicht vergleichen. Überdies sind nur 2 Schrittpferdeskelette von ihm gemessen. Er kommt zu dem Resultat, dass eine Konstanz in den Differenzen zugunsten einer Seite nicht besteht. $\mathrm{Zu}$ demselben Ergebnis führen auch meine Messungen. Tabelle VIII gibt eine Gegenüberstellung der Durchschnittsmafse des linken und rechten Metacarpus. Die Differenzen zwischen beiden sind so gering, dass sie neben den Einzelabweichungen gar nicht in Betracht kommen. Man könnte vielleicht auf gewisse Korrelationen schliessen, wenn die Differenzen nicht ganz hinter den Abweichungen zwischen den Knochen der einzelnen Paare verschwinden würden. Ebensowenig darf man aus der Übereinstimmung der Mafse 3, 4 und 5 der Laufpferde auf eine grössere Symmetrie bei den Pferden dieser Rasse gegenüber den Schrittpferden schliessen. Dazu sind die Differenzen bei denselben Mafsen der Schrittpferde viel zu gering, andere bei den Laufpferden wieder zu gross. Man kommt in bezug auf die Asymmetrie in den Malsen der Röhrbeinpaare zu dem Schluss, dass sich die grossen Differenzen zwischen den einzelnen Paaren im Durchschnitt nahezu ausgleichen und dass sie dies wahrscheinlich bei genügendem Untersuchungsmaterial ganz tun werden. Eine bedeutende Asymmetrie ist also vorhanden, aber scheinbar nicht zugunsten einer bestimmten Körperseite.

\section{Prüfung der Knochen auf ihre Widerstandsfähigkeit.}

Trotzdem die Widerstandsfähigkeit der Pferdeknochen in hippologischen Kreisen so oft erwähnt wird, ist sie doch erst in neuester Zeit einmal Gegenstand einer wissenschaftlichen Untersuchung geworden. Prof. L. HoFfMANN-Stuttgart veröffentlichte $1901^{2}$ ) einen kurzen Artikel darüber. Ausser Knochen von Pferden wurden auch solche von anderen Haustieren durch

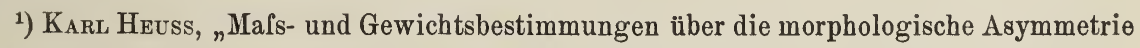
der Extremitätenknochen des Pferdes und anderer Perissodaktylen“. Paderborn 1898.

2) Berliner tierärztliche Wochenschrift 1901, No. 2. 
ihn untersucht. Die Prüfung bestand in einem Zerdrücken des ganzen Knochens der Längsachse nach und in einem Zerdrücken kongruenter zylindrischer Knochenstücke von gleichem Durchmesser und gleicher Höhe. Bezüglich der Pferdeknochen sagt L. HoffMarx: „Einen Unterschied in der Widerstandsfähigkeit habe ich nur in deren Altersverschiedenheit feststellen können, aber nicht auch in der Herkunft und Rasse." Leider ist ein ausführlicher Bericht über diese Untersuchungen bis jetzt noch nicht veröffentlicht. Sehr exakte Untersuchungen über die Knochenfestigkeit hat schon A. RAUBER 1876 veröffentlicht. ${ }^{1}$ ) Aber seine Untersuchungen erstrecken sich fast nur auf Menschenknochen. In der Hauptsache prüfte er prismatische Knochenstäbe, welche bei $80 \mathrm{~mm}$ Länge einen quadratischen Querschnitt von $2 \mathrm{~mm}$ Seitenkante hatten.

Sehr schön wäre es, wenn man bei der Prüfung der Widerstandsfähigkeit der Knochen solche Verhältnisse schaffen könnte, wie sie in Wirklichkeit den Bruch des Knochens bedingen. Das ist aber nicht möglich, schon deswegen nicht, weil wir die verschiedenen hierbei mitwirkenden Momente gar nicht genïgend kennen. Dann wäre es noch eine weitere Frage, ob wir diese Verhältnisse bei der Prüfung der Knochen künstlich schaffen könnten. Wir müssen uns deshalb begnügen, unsere Untersuchungen so zu gestalten, dass die Widerstandsfähigkeit des Knochens an sich, ohne Berücksichtigung der natürlichen Verhältnisse, zur Geltung kommt. Man kann hierbei zwei verschiedene Wege einschlagen: Entweder man prüft den Knochen als Ganzes oder man prüft einzelne Stücke desselben. Diese können wieder entweder kongruente oder äquivalente Teile der Knochen sein.

Geometrisch kongruente Stïcke werden ihrer Struktur nach selten gleichwertig sein können. Die verschiedene Stärke der Knochenwandungen bei verschiedenen Knnochen wird immer zur Folge haben, dass bei dünneren Knochenwänden der betr. Ausschnitt mehr Substanz von beiden Wandseiten enthält, während bei dickeren Wänden entweder die innere oder die äussere Wandseite mehr Material für den Ausschnitt liefert. Selbst die Stärke der Wölbung der Knochenoberfläche ist schon von Einfluss, da bei der Schaffung ebener Wandflächen der weniger gewölbte (dickere) Knochen bedeutend weniger von seiner festen Rindenschicht verliert als der stärker gewölbte (dünnere) Knochen. Glaubt man, auf die Untersuchung kongruenter Ausschnitte nicht verzichten zu können, so wird man diese von Knochen entnehmen müssen, die möglichst ähnlich gebaut sind. Solche Knochen sind aber, wie sich bei meinen Knochenmessungen ergeben hat, schwer zu finden, und es lässt sich z. B. die Wandstärke der Knochen äusserlich gar nicht erkennen.

Zweckmässiger als die Prüfung kongruenter Stücke erscheint die Untersuchung äquivalenter Teile. Diese wird um so natürlicher sein, ein je grösserer Bruchteil das betr. Stück rom ganzen Knochen ist. Deshalb sind

1) „Elastizität und Festigkeit der Knochen“ von A. RAUBER. Leipzig 1876. 
Knochenringe, welche durch rechtwinklige Schnitte zur Längsachse des Knochens gewonnen werden, sehr geeignete Versuchsstïcke. Aber mag man sie noch so hoch wählen, sie bleiben doch immer nur Teile des Knochens, und dieser ist doch ein organisches Ganzes. - Ist es uns darum auch nicht möglich, für unsere Untersuchungen die Verhältnisse zu schaffen, die beim lebenden Tiere den Bruch des Knochens verursachen, so meine ich, dürfen wir doch die Unnatürlichkeit nicht künstlich steigern dadurch, dass wir nur Ausschnitte des Knochens untersuchen. Wir prüfen den Knochen deshalb als Ganzes, was natürlich nicht ausschliesst, dass wir zur Ermöglichung unserer Experimente weniger wichtige Stücke entfernen.

Es soll nun nicht bestritten werden, dass die Prüfung kongruenter Stücke, etwa in der Weise Raubers, auch ihre Vorzüge hat. Man kann dabei mit einem gleichmässigen Material experimentieren, das weit bequemere Zahlen liefert. Aber die Untersuchung der Knochen als Ganzes hat doch so entschiedene Vorzüge, speziell auch für den Praktiker, dass ich mich für sie entschieden habe.

Weiter war nun zu erwägen, ob mit den Knochen Biege-, Knick-, Torsions- oder Zugversuche angestellt werden sollten. Eins ist mit jedem Knochen nur möglich. Ich hielt die beiden ersten für die wichtigsten und hatte anfangs den Plan, den linken Metacarpus für die Biegeversuche und den rechten für die Knickversuche zu benutzen. Doch in der Erwägung, dass die morphologische Asymmetrie beider Knochen die Resultate stark beeinflussen und so zu falschen Schlüssen führen könnte, beschränkte ich mich lieber auf eine Art der Untersuchung. Hierdurch war auch die Möglichkeit gegeben, das rechte und linke Röhrbein auf seine Leistungsfähigkeit zu vergleichen. Ich entschied mich für Biegeversuche, da ich annahm, hierdurch deutlichere Resultate als durch Knick- und andere Versuche zu gewinnen, ohne damit sagen zu wollen, dass man gerade aus Biegeversuchen den sichersten Schluss auf die Widerstandsfähigkeit des lebenden Knochens ziehen könnte.

Die gewöhnliche Form der Prüfung geschieht allerdings durch Knickversuche auf ihre rückwirkende Festigkeit in longitudinaler Richtung. Diese Prüfung wirkt aber, wie auch ZschockE anführt, ${ }^{1}$ ) keineswegs gleichmässig ein; der Druck ist nicht gleich verteilt, sondern bald auf dieser, bald auf jener Seite stärker oder schwächer, der Druck ist nicht zentriert. Dadurch erklärt es sich auch, dass es dabei nicht zur faktischen Knickung der Knochen kommt, sondern dass grössere Stücke unterhalb der Druckstelle abspringen. Die so gewonnenen Zahlen für die Druckfestigkeit sind also von der mehr oder weniger gleichmässigen Verteilung des Druckes über den ganzen Querschnitt abhängig und können nicht ohne weiteres als Mafsstab für die Widerstandsfähigkeit des gesamten Knochens betrachtet werden. Ich führe dies nur an, um es etwaigen Einwänden gegen die Beweiskraft der Biegeversuche entgegenhalten zu können. Eine völlig einwandfreie Prüfungsmethode für unsern Zweck gibt es eben nicht.

1) Zsсноске, Untersuchungen über das Verhältnis der Knochenbiegung. Zürich 1892. 
Wie schon eingangs erwähnt, führte ich die Prüfung der Knochen in dem Königl. Material-Prüfungsamt zu Gross-Lichterfelde, einem Institut der Technischen Hochschule zu Charlottenburg, aus. Dort standen mir nicht nur die geeigneten Maschinen zur Verfügung, sondern Herr Prof. Rudeloff ermöglichte mir eine korrekte Durchführung der Versuche noch dadurch, dass er mich auf alle bei der Ausführung zu erwartenden Schwierigkeiten aufmerksam machte, welche der Techniker gleich erkennt, der Laie aber erst bemerkt, wenn die Versuche bald beendet sind. Hierfür sage ich Herrn Prof. Rudeloff an dieser Stelle nochmals meinen Dank, ebenso seinem ersten Assistenten Herrn Dr. Sтоск. Zu ganz besonderem Danke bin ich noch Herrn Dipl.-Ing. Heilmann verpflichtet, welcher die technische Ausführung meiner Biegerersuche leitete und sich alle Mühe gab, meine Arbeit zu fördern.

\section{Prüfung der Biegefestigkeit.}

Die Biegeversuche wurden mittelst einer hydraulischen Presse, einer Pohlmeyer-Maschine von $50 t$ Druckkraft, ausgeführt. Die Knochen lagen

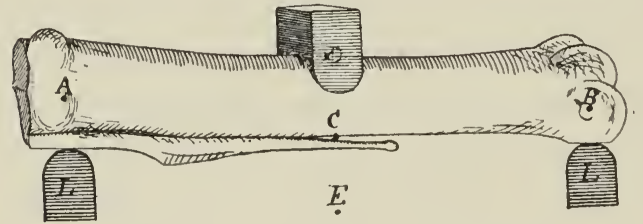

Fig. $26(1 / 4$ nat. Gr.) zeigt die Lage der Knochen bei den Biegeversuchen. $L$ die beiden Lager, $A$ und $B$ die Punkte, in welchen die Stifte zur Aufnahme des DurchbiegungsMessapparates eingesetzt wurden. $C$ der Punkt, in welchem der Taster des Messapparates einsetzte. Auf dem Knochen das Druckstück. mit den Enden auf zwei zylindrisch abgerundeten Lagern von etwa $20 \mathrm{~mm}$ Stärke. Damit die Röhrbeine fest auflagen, wurden an ihrer volaren Seite an beiden Enden durch Fräsen ebene Flächen geschaffen. Die Bruchfestigkeit des Knochens konnte dadurch nicht beeinträchtigt werden. Am meisten Masse verloren dabei die Griffelbeine.

Fig. 20 zeigt die so hergestellten Auflageflächen. Die Maschine ermöglichte es, die Lager derartig einzustellen, dass der Knochen mit beiden Enden in der ganzen Breite der abgefrästen Fläche fest auflag. Das Druckstück, welches in der Mitte quer über den Knochen gelegt wurde, war von der Form, wie Fig. 26 es zeigt. Der Radius des kreisförmigen Ausschnittes, welcher mit dem Knochen in Berührung kam, war so gross, dass das Druckstück immer nur in der Mitte, wenn auch mehrere Millimeter breit, auflag. Die untere Seite war, wie bei den Lagern, abgerundet.

Die Presse war mit einem Apparat verbunden, welcher die Grösse des sukzessive zunelımenden Druckes anzeigte. Überdies war sie so konstruiert, dass ein Absinken der Last zu erkennen war, wenn der Druck des Wassers im Knochen keinen genügenden Gegendruck mehr fand, also beim Beginn des Bruches. Aber nur selten war dies Zurückgehen zu beobachten; meist brachen die Knochen ganz plötzlich. Die beiden Hälften wurden dann mit solcher Gewalt hinweggeschleudert, dass das Anbringen von schïtzenden Drahtgeweben nötig wurde, um Verletzungen der Beobachter und Beschädigungen von Apparaten zu verhüten. Diese Vorrichtung diente gleichzeitig dazu, die einzelnen Knnochenstücke zusammenzuhalten. 
Bei der verschiedenen Länge der Knochen durfte der Abstand der Lager, die "Stützweite“, nicht immer dieselbe sein, sondern musste der jedesmaligen Röhrbeinlänge entsprechen. Diese Einstellung der Maschine liess sich bequem ausführen. Als zweckmässigste Stützweite erschien mir $86 \%$ der Röhrbeinlänge, so dass an jedem Ende $7 \%$ der Länge des Knochens über die Stützlinie hinausragte. Massgebend hierfür war, eine möglichst grosse Stützweite und eine möglichst breite Auflagefläche an beiden Enden zu gewinnen.

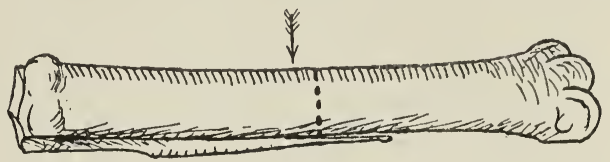

Fig. 27.

\section{Bruchformen.}

Die Form des Bruches war sehr verschieden, doch liess sich als Regel feststellen, dass der Knochen selten genau in der Mitte brach, wie man nach der Lage des Druckstückes vermuten sollte. Vielmehr trat der Bruch entweder 5-10 mm unterhalb der Mitte ein (Fig. 27) oder er war doch schräg nach dem distalen Ende gerichtet (Fig. 28). Selten verlief der Bruch schräg der proximalen Gelenkfläche zu. In der Regel sprang unter dem Druckstück, also von der dorsalen Wand des Knochens, eine flache, etwa zweimarkstückgrosse Knochenplatte los, unter welcher dann der Bruch erfolgte (Fig. 29). Dieser war manchmal so schräg bogenförmig, dass er bis in die Gelenkrolle hineinreichte (Fig. 30). Hin und wieder zersprang der Knochen auch in mehr als

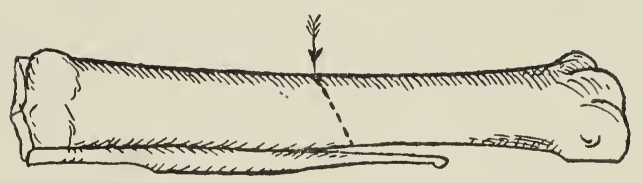

Fig. 28.

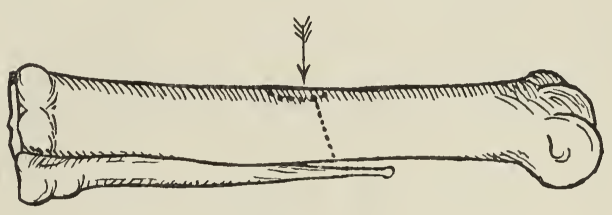

Fig. 29.

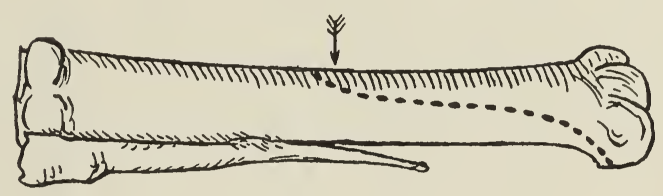

Fig. 30.

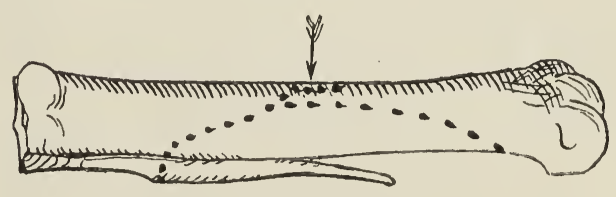

Fig. 31.

Fig. 27-31. 1/4 nat. Gr. Verschiedene Bruchformen der Knochen. Die kleinen Pfeile zeigen die Richtung des einwirkenden Druckes.

2 Hauptteile. Das geschah dann so, wie es Fig. 31 zeigt. Natürlich traten mannigfache Variationen dieser Bruchformen auf, doch waren obige Grundformen immêr zu erkennen. Meist war der Bruch ziemlich symmetrisch bezüglich der lateralen und medialen Wand. Selten stammte ein grösseres Teilstück des Knochens nur von einer Wand. Bei einigen Knochen liessen sich noch deutlich weitere Sprünge erkennen. Genauere Untersuchungen habe ich darüber nicht angestellt, doch ist anzunehmen, dass bei allen 
Knochen noch mehr oder weniger tiefe Risse entstanden, die makroskopisch nicht wahrzunehmen waren.

\section{Die Tragfähigkeit.}

Der zum Bruch des Röhrbeins nötige Druck betrug bei den Laufpferden im Durchschnitt $1159,6 \mathrm{~kg}$ und schwankte von $730 \mathrm{~kg}$ (L $36 \mathrm{r}$.) bis $1640 \mathrm{~kg}$ (L 58 l.). Bei den Schrittpferden betrug er im Mittel 1527,6 kg, war also um $368 \mathrm{~kg}$ oder $31,7 \%$ grösser. Er schwankte hier zwischen 1140 und $1900 \mathrm{~kg}$ (S 27 l. und S 9 l.).

Reihenfolge der Lauf- und Schrittpferde, geordnet nach der Tragfähigkeit nur des stärkeren Metacarpus jedes Paares.

\begin{tabular}{|c|c|c|c|c|c|c|c|c|c|}
\hline \multicolumn{6}{|c|}{ L a u f f erde: } & \multicolumn{4}{|c|}{ Schrittpferde: } \\
\hline $\begin{array}{l}\text { No. } \\
\text { L }\end{array}$ & $\begin{array}{c}\text { Bruchlast } \\
\text { in } \mathrm{kg}\end{array}$ & $\begin{array}{c}\text { No. } \\
\text { L }\end{array}$ & $\begin{array}{c}\text { Bruchlast } \\
\text { in } \mathrm{kg}\end{array}$ & $\begin{array}{l}\text { No. } \\
\mathrm{L}\end{array}$ & $\begin{array}{l}\text { Bruchlast } \\
\text { in } \mathrm{kg}\end{array}$ & $\begin{array}{c}\text { No. } \\
\mathrm{S}\end{array}$ & $\begin{array}{c}\text { Bruchlast } \\
\text { in } \mathrm{kg}\end{array}$ & $\begin{array}{c}\text { No. } \\
\text { S }\end{array}$ & $\begin{array}{c}\text { Bruchlast } \\
\text { in } \mathrm{kg}\end{array}$ \\
\hline 36 & 770 & 33 & 1120 & 16 & 1240 & 1 & 1200 & 35 & 1580 \\
\hline 29 & 940 & 41 & 1120 & 17 & 1240 & 28 & 1260 & 5 & 1600 \\
\hline 44 & 945 & 11 & 1130 & 49 & 1250 & 20 & 1270 & 29 & 1600 \\
\hline 21 & 950 & 22 & 1130 & 39 & 1255 & 27 & 1270 & 8 & 1610 \\
\hline 14 & 1000 & 48 & 1130 & 50 & 1260 & 32 & 1300 & 3 & 1630 \\
\hline 35 & 1005 & 32 & 1140 & 28 & 1270 & 12 & 1320 & 25 & 1640 \\
\hline 19 & 1030 & 57 & 1140 & 27 & 1290 & 21 & 1330 & 38 & 1640 \\
\hline 24 & 1030 & 59 & 1140 & 47 & 1310 & 14 & 1370 & 17 & 1650 \\
\hline 42 & 1040 & 3 & 1145 & 4 & 1320 & 34 & 1380 & 33 & 1660 \\
\hline 40 & 1045 & 2 & 1160 & 13 & 1320 & 30 & 1520 & 16 & 1680 \\
\hline 52 & 1050 & 26 & 1170 & 8 & 1340 & 31 & 1520 & 36 & 1700 \\
\hline 34 & 1070 & 30 & 1190 & 1 & 1350 & 7 & 1530 & 13 & 1710 \\
\hline 37 & 1070 & 10 & 1200 & 18 & 1380 & 37 & 1540 & 19 & 1710 \\
\hline 38 & 1075 & 46 & 1200 & 25 & 1380 & 15 & 1560 & 23 & 1710 \\
\hline 15 & 1080 & 31 & 1210 & 54 & 1380 & 22 & 1560 & 4 & 1790 \\
\hline 53 & 1080 & 6 & 1220 & 12 & 1540 & 24 & 1560 & 18 & 1800 \\
\hline 56 & 1090 & 9 & 1220 & 23 & 1620 & 6 & 1570 & 2 & 1850 \\
\hline 5 & 1100 & 51 & 1220 & 7 & 1640 & 11 & 1570 & 26 & 1860 \\
\hline 43 & 1100 & 45 & 1225 & 58 & 1640 & 10 & 1580 & 9 & 1900 \\
\hline 20 & 1115 & 55 & 1230 & & & & & & \\
\hline
\end{tabular}

Die grosse Differenz zugunsten der Schrittpferde findet zum Teil ihre Erklärung in der grösseren Masse der Schrittpferdeknochen. Will man aber zu einem Urteil über die Qualität der Knochensubstanz beider Pferderassen kommen, so muss man relative Zahlen gegenüberstellen.

Die Tragfähigkeit eines Körpers ist nun (abgesehen von der spezifischen Beschaffenheit seines Stoffes) abhängig von Form und Inhalt seines Querschnittes und von seiner Stützweite.

Betrachten wir zunächst den Querschnitt. Dieser beträgt (Tabelle VII) im Mittel bei den Laufpferden 806, bei den Schrittpferden 1047 qmm. Berechnen wir die Tragfähigkeit der Schrittpferderöhren auf den Querschnitt derjenigen der Laufpferde, so ergibt sich eine Tragfähigkeit von 
$1047.806=1176 \mathrm{~kg}$ gegenüber den $1159,6 \mathrm{~kg}$ der Laufpferde. Dasselbe

Verhältnis ergibt sich natürlich auch für die Tragfähigkeit pro qmm des Querschnittes. Diese beträgt bei den Laufpferden im Mittel 1432 g, bei den Schrittpferden $1456 \mathrm{~g}$ (Tabelle IX), ist also bei den letzteren immer noch um 1,67\% grösser, wenn diese Zahlen auch nicht mehr so differieren, wie die absoluten mit $31,7 \%$.

Bedeutender aber wird diese Differenz wieder, wenn wir auch das zweite Moment, die Stützweite, berücksichtigen. Da bei unseren Versuchen die Stützweite immer ein aliquoter Teil der Länge des Knochens war, so können wir bei unseren Berechnungen dafür auch die Knochenlänge selbst einsetzen. Nun beträgt die durchschnittliche Länge der Laufpferderöhren nach Tabelle VII 254,3 mm, die der Schrittpferde 257,5 $\mathrm{mm}$. Es verhält sich demnach auch die stützweite der Laufpferdeknochen zu der der Schrittpferde wie 254,3:257,5. Es waren also die Schrittpferde etwas ungünstiger bei den Biegeversuchen gestellt. Berechnen wir die Tragkraft eines $q m m$ des Querschnittes beider Rassen auf gleiche Stützweite, so muss sich das Resultat für die Schrittpferde noch günstiger stellen. Gehen wir von der Stützweite der Laufpferderöhren aus, so bleibt für diese die Zahl $1432 \mathrm{~g}$ pro $\mathrm{qmm}$ des Querschnittes bestehen, bei den Schrittpferden aber ergibt sich $\frac{1456,15 \mathrm{~g} \cdot 257,5}{254,3}=1474,47 \mathrm{~g}$ Tragkraft pro $q \mathrm{~mm}$ des Querschnittes, das übertrifft die Laufpferde um 2,95\%.

Dies günstige Resultat ergibt sich für die Schrittpferde, trotzdem die Form ihres Knochenquerschnittes für die Biegeversuche nicht so günstig ist, wie die der Laufpferde. Bekanntlich steigert nicht nur die Grösse, sondern auch die Form des Querschnittes die Tragfähigkeit. Die Leistungsfähigkeit des Querschnittes wächst einfach mit der Grundlinie, aber im Quadrat mit der Höhe, so dass bei gleichem Flächeninhalt der höhere Querschnitt immer der tragfähigere ist. Nun sind aber die Röhren der Laufpferde höher gebaut als die der Schrittpferde (Tabelle VII Seite 6, vergl. auch die idealen Querschnitte Fig. 1). Es waren also die Laufpferde in der Form des Querschnittes günstiger gestellt und haben doch eine geringere relative Tragkraft gezeigt.

Aber es kommt noch ein ferneres Moment hinzu, welches für die Qualität der Schrittpferdeknochen spricht. Bei der vorstehenden Berechnungsweise ist der Querschnitt immer als geschlossene Fläche betrachtet, die Markhöhle also gar nicht berücksichtigt. Das wäre gerechtfertigt, wenn der Anteil der Markhöhle am Querschnitt, oder was dasselbe ist, die relative Wandstärke bei beiden Rassen die gleiche wäre. Nun ist aber nach den Resultaten meiner Messungen die durchschnittliche Wandstärke der Schrittpferde relativ geringer als die der Laufpferde, nämlich um $9 \%$ (Tabelle VI Reihe 12). Bei Berücksichtigung auch dieses Momentes würden also die Zahlen für die Leistungsfähigkeit des Schrittpferdeknochens noch günstiger werden. 
Ich verzichte darauf, diese für die Schrittpferde günstigeren Zahlen, welche sich bei gleicher Form des Querschnittumrisses und gleicher relativer Wandstärke ergeben würden, zu berechnen. Man möchte sonst geltend machen, dass die durch die faktischen Messungen festgestellten Differenzen zu gering wären, als dass man mit diesen Zahlen weiter operieren könnte.

Nun mag mancher diese ganze Berechnungsmethode der Biegefestigkeit für zu kompliziert halten. Der Praktiker hat gern einfache, anschauliche Vergleiche. Ich habe darum die Tragfähigkeit noch verglichen nach dem Volumen, indem ich dieselbe für $1 \mathrm{ccm}$ des Knochens (diesen massiv angenommen) berechnete. Das Resultat ist folgendes: $1 \mathrm{ccm}$ der Laufpferderöhre trägt im Mittel $411,13 \mathrm{~kg}, 1 \mathrm{ccm}$ der Schrittpferderöhre dagegen $417,51 \mathrm{~kg}$. Also auch hier schneiden die Schrittpferde günstiger ab, trotz ihrer auch relativ grösseren Markhöhle, wenn auch nur um 1,56\%.

Hier wird vielleicht eingewandt, dass die für die Schrittpferde günstigeren Zahlen durch den grösseren Prozentsatz von Wallachen verursacht seien, da diese stärkere Knochenwände besässen. Demgegenüber stelle ich noch einmal die betreffenden Zahlen zusammen:

\begin{tabular}{c|c|c}
\hline $\begin{array}{c}\text { Die durchschnittl. Wandstärke } \\
\text { beträgt vom mittleren Radius } \\
\text { des Querschnittes: }\end{array}$ & $\begin{array}{c}\text { Bei Lauf- } \\
\text { pferden } \\
\%\end{array}$ & $\begin{array}{c}\text { Bei Schritt- } \\
\text { pferden } \\
\%\end{array}$ \\
\hline Bei Stuten und Wallachen zu- & & \\
sammen . . . . . . . . . . & 53,9 & 49,1 \\
"Wallachen allein . . . . . . . & 54,7 & 49,6 \\
" Stuten allein . . . . . . . . & 52,8 & 47,2
\end{tabular}

Die Wandstärke der Wallachen unter den Schrittpferden bleibt immer noch geringer als die Wandstärke der Stutenknochen unter den Laufpferden. Man wird also die ermittelte grössere Leistungsfähigkeit der Schrittpferdeknochen nicht durch die grössere Zahl von Wallachen erklären können.

\section{Die Durchbiegung der Knochen.}

Bei den Biegeversuchen wollte ich nicht nur die Grösse des Druckes messen, welcher nötig war, den Knochen zu zerbrechen, sondern es sollte gleichzeitig der Grad der Durchbiegung festgestellt werden, um hieraus auf die Elastizität der Knochen schliessen zu können. Diese Feststellung hat die meisten Umstände, den grössten Zeitaufwand bei meinen ganzen Untersuchungen verursacht, und doch muss ich gestehen, dass ich den beabsichtigten Zweck nicht erreichte. Ein klares Urteil über die Elastizität der Laufpferdeknochen gegenüber der der Schrittpferde lässt sich aus den erhaltenen Zahlen nicht gewinnen. Ich beschränke mich deshalb auf die Wiedergabe der Prüfungsergebnisse, ohne weitere Erwägungen daran zu knüpfen. Diese Zahlen erwecken immerhin so viel Interesse, dass ihre 
Mitteilung hier gerechtfertigt erscheint, und sie können bei späteren Untersuchungen vielleicht als Material verwandt werden.

Die Feststellung der Durchbiegung erscheint zunächst sehr einfach, als wäre es nur nötig, die Abstandsverringerung des Punktes $D$ oder $C$ des Knochens Fig. 26 von dem senkrecht darunter liegenden Punkt $E$ der Maschine zu messen. Nun wird aber der Knochen durch den Druck in $D$ so stark auf die Lager gepresst, dass man annehmen muss, dass die beiden Lager in den Knochen etwas eindringen, oder anders gesagt: dass die Punkte $A$ und $B$ sich den Lagern nähern. Die Abstandsverringerung zwischen $D$ (resp. $C$ ) und $E$ ist demnach nicht reine Durchbiegung, sondern Summe von Durchbiegung und Verdrückung an den Auflagestellen. Da die Auflageflächen der Knochen breit hergerichtet wurden, kann das Hineinpressen des Knochens in die Lager ja nicht bedeutend sein, muss aber, weil es sich bei der Durchbiegung immer nur um Hundertstel Millimeter handelt, berücksichtigt werden. Um die durch das Hineinpressen der Lager in den Knochen entstehenden Ungenauigkeiten auszuscheiden, muss die Lageveränderung der Punkte $D$ resp. $C$ zu festen Punkten innerhalb des Knochens selbst festgestellt werden. Darin lag die Schwierigkeit des Experimentes.

In jeden Knochen wurden auf einer Seite in den Punkten $A$ und $B$, welche um die Stützweite

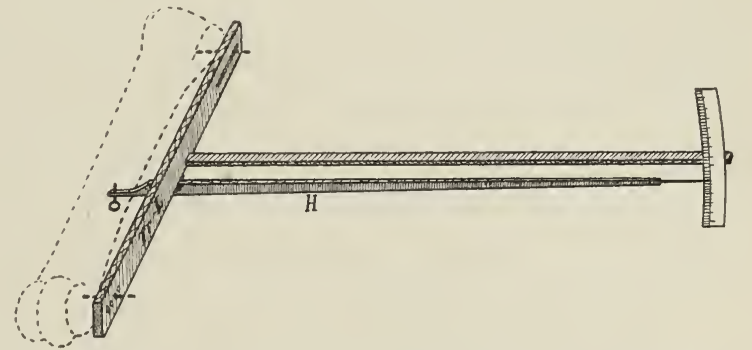

Fig. 32. ca. $1 / 6$ nat. Grösse. Hebelapparat zur Feststellung der faktischen Durchbiegung. $\operatorname{Tr} L$ Tragleiste, auf die beiden Messingstifte des Knochens gestreift, $H$ Hebel, beweglich an der Tragleiste aufgehängt, links mit dem unter den Knochen passenden Taster, rechts auf einer Skala die Grösse der Durchbiegung anzeigend. des Knochens entfernt waren, also senkrecht über den Scheiteln der beiden Lager lagen, zwei etwa $2 \mathrm{~mm}$ starke und ca. $30 \mathrm{~mm}$ herausragende Messingstifte so eingesetzt, dass sie senkrecht zur Längsachse des Knochens und parallel zu den Auflageflächen standen, bei dem aufliegenden Knochen also horizontal nach der Seite gerichtet waren. An diesen Stiften wurde nun ein Apparat befestigt, welcher die Durchbiegung des Punktes $C$ zur Linie $A B$ anzeigte, wodurch die Lageveränderung des Knochens zur Maschine eliminiert war.

Dieser Apparat wurde nach Angaben des Herrn Prof. Rudelofr konstruiert und ist in Fig. 32 dargestellt. Die Tragleiste " $\operatorname{Tr} L^{\text {" war }}$ an einem Ende mit einem Schlitz, am anderen mit mehreren Löchern versehen, womit sie auf die Messingstifte des Knochens aufgestreift werden konnte. Diese Einrichtung ermöglichte die Befestigung bei jeder Entfernung der Stifte, der verschiedenen Stützweite entsprechend. Der bewegliche Hebelarm $H$ war durch ein Scharnier beweglich an der Tragleiste befestigt. Sein kürzerer Arm war so abgeschrägt, dass er bequem unter jeden Knochen 
fasste. Ein verschiebbarer Messingstift an seinem Ende diente als Taster. Die an einem langen, festen Arm angebrachte Skala zeigte Millimeterteilung. Die Durchbiegung wurde nach je $100 \mathrm{~kg}$ Belastung abgelesen. Aus den angezeigten Mafsen wurde dann die faktische Durchbiegung berechnet.

So zweckmässig der Apparat konstruiert war, schien er doch nicht ordentlich zu funktionieren. Er zeigte bei den einzelnen Druckstufen so unregelmässige Durchbiegungen an, wie sie nicht zu erwarten waren. Oft waren die Schwankungen so gross, dass ich glaubte, sie nicht passieren lassen zu dürfen, und bei manchen Knochen wurden deshalb die Biegeversuche wohl dreimal begonnen. Ein Grund für die Unregelmässigkeiten war vorläufig nicht zu finden; darum liessen sie sich auch nicht abstellen.

Ein anderer Übelstand war der notwendig leichte Bau des Apparates, der es nötig machte, den Apparat vor dem Bruch des Röhrbeins jedesmal abzunehmen, wenn er nicht zertrümmert werden sollte. Dies Abnehmen (während der Knochen in der Maschine belastet war) war immer mit Schwierigkeiten verknüpft. Manchmal überschätzte man auch die Tragkraft des Knochens und der Bruch trat ein, ehe der Apparat entfernt war. Dann waren wieder langwierige Reparaturen nötig. Einmal musste sogar der Apparat gänzlich erneuert werden. Der Hauptfehler war aber der, dass man wegen der vorherigen Entfernung des Apparates die Durchbiegung innerhalb der letzten Druckstufen, die doch gerade die wichtigsten Zahlen erwarten liessen, nicht feststellen konnte.

Deshalb entschloss ich mich, nachdem ich bei etwa 80 Knochen diesen Nessapparat angewandt hatte, ihn nicht weiter zu benutzen, sondern einen stabileren Messapparat anzubringen. Dieser wurde nicht am Knochen, sondern an der Maschine befestigt, so dass ich die oben erwähnten, durch das Hineinpressen der Lager in den Knochen entstehenden Ungenauigkeiten in den Kauf nehmen musste. Doch war anzunehmen, dass sich diese bei allen Knochen annähernd ausgleichen würden. Nun konnte ich schneller arbeiten und brauchte bei keinem Knochen mehrere Male zu beginnen, was doch auch die Widerstandsfähigkeit der Knochen beeinflussen konnte. Die Durchbiegungsschwankungen wollten allerdings doch nicht verschwinden, wodurch ich darauf hingewiesen wurde. den Grund dafür in den Knochen selbst zu suchen. Es war nun möglich, auch die Durchbiegung der letzten Druckstufen zu messen. Dafür konnte allerdings mit diesem Apparat wieder nicht die Durchbiegung der ersten Druckstufe festgestellt werden. Aber gerade diese hat nur geringen Wert, da es sich bei Gebrauch des ersten Apparates gezeigt hatte, dass die Zahlen für die Durchbiegung der ersten Druckstufe weitaus am meisten über die wahrscheinliche Durchbiegung hinausgehen. Das mag daher kommen, dass sich bei Beginn des Druckes der Knochen erst den Lagern und dem Druckstück fest anschmiegt. Dieser neue Apparat zeigte die Durchbiegung in $1 / 500 \mathrm{~mm}$ an, während die beiden in Fig. 32 dargestellten eine etwa 8 resp. 9 fache Übersetzung hatten. 
In den Tabellen habe ich die Durchbiegung daraus in $1 / 100 \mathrm{~mm}$ angegeben, und zwar in Druckstufen von je $100 \mathrm{~kg}$. Einige Male wurde das Ablesen verpasst. Dann gibt die betr. Zahl der Tabelle die Grösse der Durchbiegung für die beiden Druckstufen an.

Die Haupttabelle zeigt die gewonnenen Zahlen. Trotz der oben erwähnten Schwankungen ist deutlich ersichtlich, wie im allgemeinen die Durchbiegung in den höheren Druckstufen eine grössere wird. Bei der tabellarischen Eintragung habe ich die Unregelmässigkeiten der Durchbiegung nicht auszugleichen gesucht, sondern habe sie genau wiedergegeben. Die Knochen der Laufpferde No. 17, 20, 23, 25, 26 und 29 konnten wegen mangelnder Zeit auf ihre Durchbiegung nicht geprüft werden. Ich habe dann wenigstens, um das Material zu verwerten, die Bruchlast festgestellt.

Aus den so gewonnenen Zahlen galt es nun, zu einem Urteil über die Elastizität der Laufpferd- und Schrittpferdröhren zu gelangen. Auf die Feststellung der Gesamtdurchbiegung der einzelnen Knochen glaubte ich im Hinblick auf die Unregelmässigkeiten innerhalb der ersten Druckstufen verzichten zu müssen. War es doch fraglich, ob die gewonnenen Zahlen wirklich nur von der Durchbiegung der Knochen herrührten, und ob nicht ein anderes Moment mitwirkte. Ich werde hierauf im Abschnitte über die Ursachen der unregelmässigen Widerstandsfähigkeit der Knochen noch näher eingehen.

Einwandfreiere Vergleichszahlen als die der Gesamtdurchbiegung glaubte ich zu bekommen, wenn ich nur die Durchbiegungssumme der zweiten Hälfte aller Druckstufen berücksichtigte. Die Zahlen hierfür (also für die zweite Hälfte allein) habe ich in der Haupttabelle angegeben. Bei ungerader Zahl der Druckstufen wurde die mittlere zur Hälfte mitgerechnet. Da die Knochen selten gerade dann brachen, wenn ein volles neues Hundert von Kilogrammen Druck erreicht war, so wurde die Durchbiegung für die über das letzte volle Hundert hinausgehende Druckkraft nicht mehr festgestellt. Es wurde z. B. für den Knochen L $41 \mathrm{r}$,, der erst bei $1095 \mathrm{~kg}$ Belastung brach, die letzte Durchbiegung bei $1000 \mathrm{~kg}$ Belastung mit 55 Hundertsteln Millimeter abgelesen. Danach ertrug der Knochen noch eine Mehrbelastung von 95, also fast $100 \mathrm{~kg}$, für welche die Durchbiegung nicht mehr gemessen wurde. Diese Durchbiegung innerhalb der letzten angefangenen Druckstufe durfte bei der Feststellung der "Gesamtdurchbiegung“" nicht übersehen werden. Ich suchte sie deshalb zu berechnen. Als. Anhalt hierfür dienten mir diejenigen Knochen, welche gerade während der Ablesung, also am Ende einer Druckstufe gebrochen waren. Bei diesen war die Durchbiegung bis zur Grenze festgestellt und die Betrachtung der Durchbiegung der letzten Druckstufe dieser Knochen liess es tunlich erscheinen, diese Grösse bei den übrigen Knochen für die über den letzten vollen Hunderter überschiessende Belastung nach folgender Formel festzustellen:

$\frac{\text { Summe der Durchbiegung } \times 2 \times \text { überschiessende Belastung }}{\text { Zahl der Druckstufen } \times 100}$, also für den erwähnten Knochen L 41 r. $\frac{207 \cdot 2.95}{5.100}=79$. Die so gewonnenen Zahlen wurden 
dann der Summe der Durchbiegung der letzten Hälfte der Druckstufen hinzugezählt, und es ergab sich so die „Gesamtdurchbiegung“.

Einwandfrei ist diese Berechnungsweise zwar nicht; aber sie dürfte genügen, ein ungefähres Bild der Elastizität der Knochen zu geben. Natürlich konnten die Röhrbeine, bei welchen die Durchbiegung mit dem in Fig. 32 wiedergegebenen Messapparate festgestellt war, nicht zur Berechnung dieser „Gesamtdurchbiegung“" benutzt werden, da hier die Durchbiegung der letzten und wichtigsten Druckstufen nicht gemessen war. Die Durch-

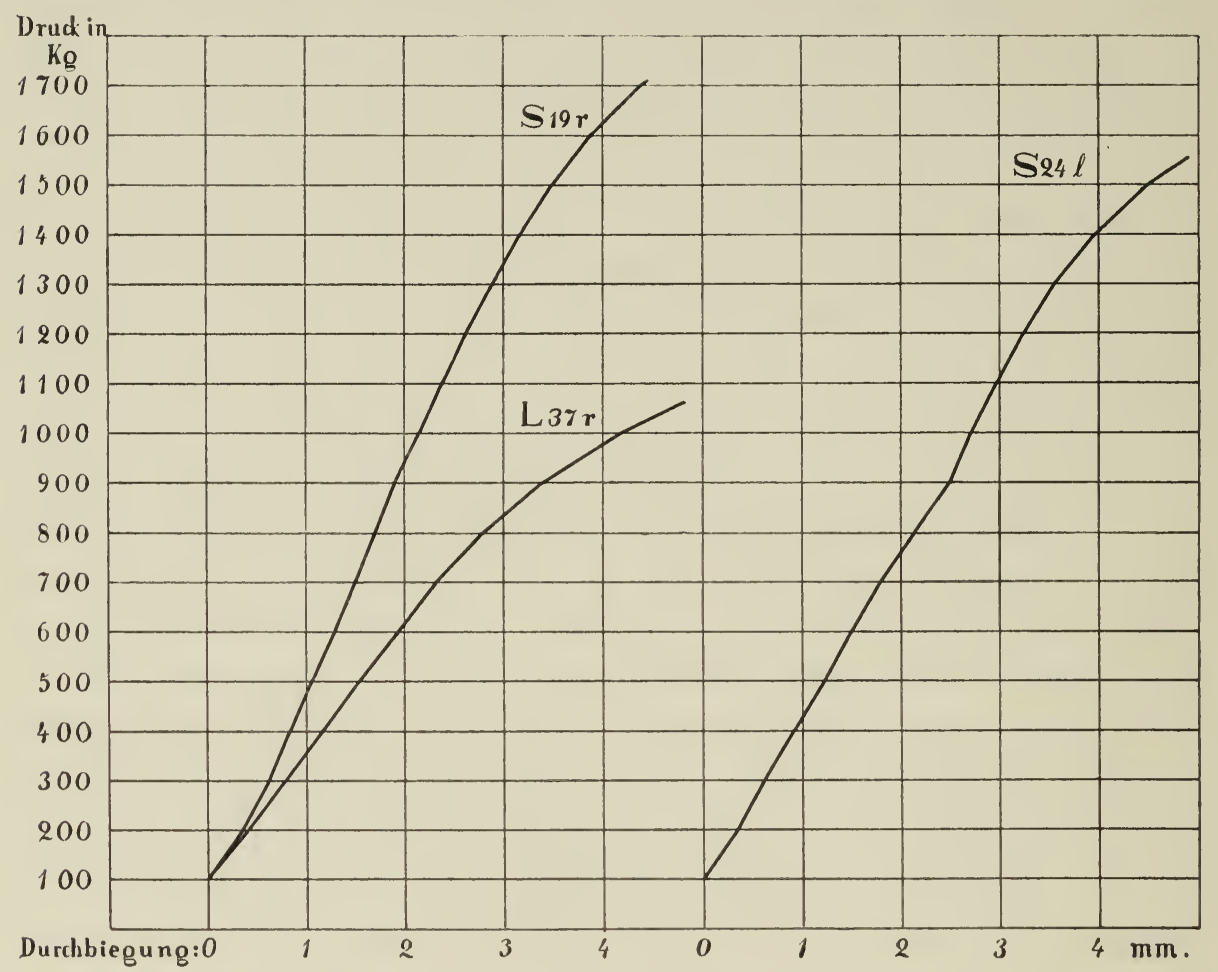

Fig. 33. Biegungskurven dreier Röhrbeine. S 19 r. und L 37 r. normal verlaufend, d. h. während der ersten Hälfte der Belastung unregelmässig, danach gleichmässig nachgebend. S $24 \mathrm{l}$. bis zum Druck von $900 \mathrm{~kg}$ normal (unregelmässig), dann plötzlich stärkeren Widerstand leistend.

schnittszahlen beruhen deshalb nur auf Messungen an 36 Laufpferden und 20 Schrittpferden.

Eine Zusammenstellung der Resultate gibt Tabelle X. Aus dieser geht hervor, dass die Durchbiegung bei den Schrittpferden trotz der grösseren Zahl der Druckstufen und trotz der grösseren Stützweite eine geringere als bei den Laufpferden ist. Der Grund hierfür ist aber meines Erachtens nicht ein spröderes Material der Schrittpferdeknochen, vielmehr findet diese Erscheinung in der Verschiedenheit von Form und Inhalt des Querschnittes eine genügende Erklärung.

Um von der verschiedenen Durchbiegung der Knochen ein anschauliches Bild zu geben, füge ich in Fig. 33 die Biegungskurven einiger Knochen 
bei. Diese zeigen deutlich die Unregelmässigkeit der Durchbiegung in den ersten Druckstufen und die Zunahme derselben zum Schlusse hin. Das auch zur Anschauung gebrachte abnorme Verhalten des Knochens S 241. werde ich noch weiterhin erwähnen.

\section{Asymmetrie der Leistung. (Tabelle $\mathrm{XI}$ ).}

Eine Gegenüberstellung der Leistungsfähigkeit der linken und rechten Röhrbeine gibt Tabelle XI. Diese zeigt, dass sowohl nach Tragfähigkeit als auch nach Biegsamkeit bei beiden Gruppen das linke Röhrbein dem rechten überlegen ist, sowohl absolut als auch relativ. Die Differenzen sind aber nur gering und gelten auch nur für den Durchschnitt. Der Unterschied in der Leistungsfähigkeit zwischen den beiden Knochen eines zusammengehörigen Paares ist meist bedeutender; oft ist er so gross, dass die durchschnittlichen Differenzen daneben ganz verschwinden. Das zeigen die Zahlen für die absoluten Leistungen einiger Knochen, welche in der Tabelle beigegeben sind.

Vergleicht man die Asymmetrie der Leistung (Tabelle X) mit der der Form (Tabelle VIII), so sucht man vergebens nach einer Parallele. Das liegt wohl in der Geringfügigkeit der Differenzen in beiden Tabellen begründet und führt zu der Annahme, dass diese geringen Unterschiede nur zufällige sind, die wahrscheinlich bei Behandlung noch grösseren Materials immer mehr verschwinden.

Wollte man aus den geringfügigen Differenzen einen Schluss über das Verhältnis von Leistungsfähigkeit und Form wagen, so würde man sagen müssen, dass das stärkere (linke) Röhrbein im Durchschnitt ein etwas geringeres Volumen, geringere oder gleiche Länge, flacheren Bau und ein höheres oder gleiches spezifisches Gewicht hat. Auffallend ist hierbei vor allem, dass ein flacherer Bau für die Tragfähigkeit günstig sein soll, während sonst allgemein das Gegenteil der Fall ist. Sieht man aber, dass der Unterschied in der Höhe des Querschnittes nur 0,3 resp. 0,4\% beträgt, so wird man dadurch noch mehr in der Annahme bestärkt, dass diese kleinen Differenzen auf Messungsfehlern oder Zufälligkeiten beruhen werden.

\section{Vergleich nur der stärkeren Knochen der einzelnen Röhrbeinpaare (Tabelle XII).}

Die grossen Differenzen in der Widerstandsfähigkeit zwischen den beiden zusammengehörigen Knochen einzelner Röhrbeinpaare kann zu der Vermutung führen, dass manche der schwächeren Knochen pathologisch verändert seien. Vielleicht wäre es daher richtiger gewesen, nur den jedesmal leistungsfähigeren Knochen jedes Paares bei den Berechnungen zu berücksichtigen. Doch habe ich zunächst lieber mit den Zahlen sämtlicher Knochen operiert, um das zur Verfügung stehende mühsam gesammelte Material möglichst auszunutzen. Danach füge ich nun in der Tabelle XII eine Zusammenstellung der Durchschnittszahlen über die Leistungsfähigkeit nur des stärkeren von beiden Röhrknochen an. ${ }^{1}$ ) Zum bequemeren Ver-

1) Aus denselben Erwägungen sind schon in den kleinen Tabellen Seite 8, 9 und 32 nur die Zahlen für das stärkere Röhrbein gegeben. 
gleich habe ich gleich die allgemeinen Durchschnittszahlen aus Tabelle IX und $\mathrm{X}$ daneben gestellt und in jedem Falle die Differenz der Leistung zwischen Lauf- und Schrittpferden in Prozenten beigefügt.

Aus dieser Zusammenstellung geht hervor, dass das Verhältnis in der Leistungsfähigkeit zugunsten der Schrittpferde ungefähr dasselbe geblieben ist. Nur die Differenz in der Tragkraft für jeden Kubikzentimeter des Volumens hat sich erheblich verringert, auf ein Drittel der allgemeinen Durchschnittsdifferenz; doch geht auch hier noch das Schrittpferd dem Laufpferd in der Leistungsfähigkeit voran.

\section{Korrelation von Form und Leistung.}

Wie die Abschnitte über Asymmetrie in Form und Leistung gezeigt haben, ist es schwierig, eine Parallele zwischen beiden $\mathrm{zu}$ finden. Ich wollte aber doch die Möglichkeit geben, irgendwelche Korrelationen herauszufinden und habe deshalb in den Tabellen XIII L und S die stärkeren Röhrbeine der einzelnen Paare noch einmal zusammengestellt. Es sind aber nicht die einzelnen Mafse und Leistungen der Knochen aufgeführt, sondern jedes Röhrbein hat in jeder Spalte diejenige Ordnungsnummer erhalten, welche seinem Mals oder seiner Leistung in der betr. Spalte zukommt. Aus der Tabelle ist also z. B. zu ersehen, dass das stärkere linke Röhrbein des Laufpferdes No. 1 nach dem absoluten Gewicht das 40., nach dem Volumen das 36. ist usw. Da es dasselbe spezifische Gewicht hat wie das Röhrbein von L 13, so mussten beide den gleichen Rang erhalten. Da ferner aber zwei Knochen auch zwei Nummern haben müssen, wurden beide mit den Zahlen „51 und 52" bezeichnet. Aus denselben Erwägungen wurden auch in der Rubrik "Gesamtdurchbiegung“" bei den Laufpferden, die in dieser Spalte nur 36 Nummern enthalten, die Zahlen 1-59, bei den Schrittpferden statt der vorhandenen 20 Nummern die Zahlen 1 -38 verwandt, weil ja sonst kein Vergleich mit den übrigen Spalten möglich war. Dadurch, dass in regelmässigen Zwischenräumen einige Zahlen ausgelassen wurden, konnte dies durchgeführt werden. Nach demselben Grundsatz ist auch in den beiden Spalten für die Wandstärke verfahren, wo auch einige Zahlen fehlen.

Zur Vervollständigung dieser Tabelle ist das Verhältnis der Knochenstärke zum über der Haut gemessenen Röhrbeinumfang und das Alter mit aufgeführt.

Fast genau gleichlaufend müssen in dieser Zusammenstellung natürlich die Spalten „mittlerer Durchmesser" und „Flächeninhalt des Querschnittes" sein, ebenso „absolutes Gewicht" und „Volumen“. Auch die beiden Spalten für die relative Tragfähigkeit gehen einigermassen parallel. Aber eine weitere deutliche Parallele herauszufinden, ist mir nicht möglich gewesen. Das schliesst aber nicht aus, dass eine solche Parallele zwischen Form und Leistung doch vorhanden ist und nur dadurch verdeckt wird, dass immer mehrere Formmomente als Faktoren gemeinsam die Leistung beeinflussen. 


\section{Ursachen der unregelmässigen Widerstandsfähigkeit.}

Die Widerstandsfähigkeit der Knochen zeigt in zweifacher Hinsicht eine gewisse Regellosigkeit: erstens in der Zunahme der Durchbiegung innerhalb der einzelnen Druckstufen, zweitens in der scheinbaren Unabhängigkeit der Tragkraft vom morphologischen Bau, wie sie besonders durch die Differenz der Tragkraft der beiden Knochen zusammengehörender Paare, die keine äusserliche Verschiedenheit zeigen, zum Ausdruck kommt. Ganz allgemein liesse sich das ja dadurch erklären, dass ein frischer Knochen ein organisches Gebilde ist, das sich nicht so einfach, wie ein toter, homogener Körper verhält. Doch befriedigt diese Erklärung nicht recht, und man muss versuchen, obige Erscheinungen weiter auf ihre Ursachen zu verfolgen.

Ich gehe aus von der unregelmässigen Durchbiegung innerhalb der ersten Druckstufen. Wie schon im Abschnitt „Durchbiegung“ angedeutet, kommt der Gedanke, dass die gemessene Grösse der Durchbiegung nicht nur verursacht sei durch die eigentliche Biegung des Knochens, sondern ausserdem noch durch andere Momente, etwa durch Torsion der Diaphyse gegenüber den aufliegenden Enden. Die verschiedenen Torsionslagen würden dann dem Druck verschiedene Widerstände bieten, wodurch die Unregelmässigkeiten in den ersten Druckstufen erklärt wären. Da in der zweiten Hälfte der Laststufen die Durchbiegung eine regelmässige Zunahme zeigt, müsste weiter angenommen werden, dass Torsionsänderungen bei höherem Druck nicht mehr vor sich gehen.

Aber das Verhalten eines der untersuchten Knochen machte es doch unwahrscheinlich, dass Torsionen die Ursachen für die Unregelmässigkeit der Durchbiegung seien. Es war das der linke Metacarpus von S 24, einem etwa zwölfjährigen, belgischen Wallach. Wie die Haupttabelle zeigt, stieg die Durchbiegung während der ersten 9 Druckstufen etwas unregelmässig von 29 auf 36 Hundertstel Millimeter innerhalb jeder Stufe. Dann aber geht die Grösse der Durchbiegung in der 10. Stufe ganz plötzlich auf nur 23 Hundertstel zurück, um nun regelmässig bis zum Bruche zuzunehmen. In Fig. 33 ist die Biegungskurve dieses Knochens mit dargestellt. Daraus sind deutlich die beiden Biegungsperioden des Knochens erkennbar. In der ersten Periode ist der Knochen ziemlich biegsam und wird dann ganz plötzlich in der zweiten widerstandsfähiger, ein Verhalten, welches dem gewöhnlichen Verhalten homogener Körper gerade entgegengesetzt ist. Kein anderer Knochen zeigt ähnliche Erscheinungen, sondern immer nur unregelmässige Schwankungen, niemals eine zweite widerstandsfähigere Periode. Noch auffallender wurde das Verhalten des Knochens S 241. dadurch, dass am Schluss der 9. Druckstufe ein lautes Krachen ertönte, so dass die Beobachter glaubten, der Knochen wäre gebrochen und nicht wenig erstaunt waren, als er nun gerade erst seine grösste Widerstandsfähigkeit zeigte. Wie soll man sich das erklären! Das Krachen war doch sicher verursacht durch irgend welche Verbandslockerungen im Innern des Knochens. 
Dass die auffallenden Durchbiegungszahlen verursacht seien durch ungenaues Arbeiten des Messapparates, ist ausgeschlossen. Bei diesem Knochen wurde nicht mit dem Hebelapparat (Fig. 32) gearbeitet, sondern mit dem später gebrauchten stabilen. Auch kann die hydraulische Presse unmöglich fehlerhaft funktioniert haben. Dass die Erscheinung durch den Knochen selbst hervorgerufen war, ist ja auch durch das Krachen bewiesen.

Das liesse sich auf folgende Weise erklären: Der Knochen ist kein toter, homogener Körper, dessen einzelne Fasern bei der Durchbiegung gleichzeitig und regelmässig wirksam sind. Vielmehr ist er ein organisches Gebilde von sehr unregelmässiger Form, so dass es wahrscheinlich ist, dass seine einzelnen Elemente (die Haversschen Kanäle, die Grund- und die Speziallamellen) zu verschiedenen Zeitpunkten und durchaus regellos beansprucht werden. Die Folge davon muss sein, dass immer nur ein Teil der Gesamtheit der Knochenelemente dem Biegedruck Widerstand leistet. Zerreissen nun einige Elemente der gerade beanspruchten Gruppe durch Überlastung, so werden sich die bleibenden weiter durchbiegen müssen und andere Knochenelemente werden mit beansprucht. Es kann nun geschehen, dass so viel neu mitwirkende Elemente hinzutreten, dass die jetzt zusammenwirkende Gruppe kräftiger ist, als die vorher wirksame, und die Folge wird sein, dass die Durchbiegung in der neuen Druckstufe eine geringere ist, als die in der vorigen. Mit zunehmendem Druck aber werden nach und nach alle Knochenelemente in Anspruch genommen und von dem Augenblick an, wo der Knochen mit seiner vollen Widerstandsfähigkeit einsetzt, muss die Durchbiegung eine regelmässig zunehmende werden. Dieser Zeitpunkt scheint den vorliegenden Versuchen nach immer etwa bei halber Belastung, also in der Mitte der Laststufen einzutreten.

Hiernach wäre also die unregelmässige Durchbiegung der untersuchten Knochen in den ersten Druckstufen eine notwendige Folge dieser ungleichzeitigen Inanspruchnahme der einzelnen Knochenelemente, und es war demnach ein aussichtsloses Bemühen, einen Apparat konstruieren zu wollen, der eine gleichmässig ansteigende Durchbiegung der Knochen angezeigt hätte, weil eine solche eben nicht vorkommt.

Findet eine solche ungleichzeitige Inanspruchnahme der Knochenelemente wirklich statt, so kann der Fall eintreten, dass anfangs eine grössere Gruppe von Knochenelementen gar nicht beansprucht wird, während eine schwächere Gruppe den ganzen anfänglichen Druck in der Hauptsache aufzunehmen hat. Da muss aber der Augenblick kommen, wo diese schwächere Gruppe nachgibt, vielleicht alle ihre Elemente gleichzeitig; und die dadurch bedingte stärkere Durchbiegung lässt nun die so lange ruhende Hauptgruppe in Funktion treten. Diese setzt einen grösseren Widerstand entgegen, gestattet also nur eine geringere Durchbiegung als die anfängliche war. So liesse sich das Verhalten des Knochens S 241. erklären. Diese Annahme bedingt durchaus nicht, dass jede der beiden erwähnten Gruppen dieses Knochens in sich ganz gleichartig wirkende Elemente enthielte, Innerhalb jeder Gruppe können vielmehr wieder Schwan- 
kungen durch die ungleichzeitige Wirksamkeit ihrer Elemente stattfinden. Auch ist es wahrscheinlich, dass neben diesen beiden Gruppen noch eine dritte Gruppe bestand, welche sowohl mit der ersten als auch nach deren Nachgeben mit der zweiten gemeinsam wirkte.

Das Krachen des Knochens trat demnach ein, als die schwächere erste Gruppe plötzlich zerriss. Übrigens wurde ein schwächeres Knacken öfter bei den Versuchen vernommen. Die Beobachter nahmen dann als mögliche Ursache an, dass sich die Griffelbeine vom Metacarpus III gelöst hätten, was sich aber nicht kontrollieren liess. Jetzt halte ich es für das Wahrscheinlichere, dass ähnlich wie bei S 24 l. in dem Augenblick des Knackens eine Gruppe von Knochenelementen plötzlich zerriss. Leider beachtete ich diese Erscheinung so wenig, dass ich sie nicht notierte.

Nach diesen Ausführungen würden demnach bei eintretender Beanspruchung des Metacarpus nicht gleich alle Teile desselben ihren Widerstand geltend machen, sondern je nach der Art der Beanspruchung immer nur einzelne Gruppen, wenn es nicht gerade zum Bruch kommt.

Diese gruppenweise Wirksamkeit der Knochenelemente scheint man aber allgemein für ausgeschlossen zu halten. Wenigstens sagt Zsch0скE, der die einschlägige Literatur in weitestem Malse berïcksichtigt: „Dass der für grosse Anforderungen genügend entwickelte Knochen auch für kleinere genügt und für diese keine Spezialstruktur zu besitzen braucht, ist selbstverständlich." - Eine eigentliche Spezialstruktur für geringere Beanspruchung hat allerdings der Knochen auch nach der oben vorgetragenen Annahme nicht. Die ungleichzeitige und darum gruppenweise Inanspruchnahme der einzelnen Knochenelemente aber ist in der Funktion einer solchen Spezialstruktur gleichwertig.

Eine Bestätigung meiner oben ausgeführten Annahme der gruppenweisen Wirksamkeit der Knochenelemente scheint mir in den Beobachtungen RaUbERS zu liegen. Wie schon erwähnt, prüfte RaubER prismatische Knochenstäbe von $80 \mathrm{~mm}$ Länge. Die für die Biegungsversuche benutzten Stäbchen entstammten verschiedenen Röhrenknochen von Mensch und Rind. Rauber stellte bei der Durchbiegung der Stäbchen zwei Phasen fest. Er sagt: ${ }^{1}$ ) „Die Grösse der Formänderung, bis zu deren Erreichung jene Proportionalität (direkt Kubus der Länge, indirekt der Breite und indirekt dem Kubus der Dicke) stattfindet, schwankt im übrigen so sehr, dass es unmöglich ist, sie genauer zu bezeichnen als damit, dass sie häufig noch jenseits der Elastizitätsgrenze liege. Eine weit grössere Reihe von hierauf gerichteten Versuchen, als die zu diesem Zweck mitgeteilten, lässt eine genauere Bestimmung infolge noch unregelmässigeren Verlaufes ebensowenig zu; sie wurden darum nicht mitgeteilt. Auf diese proportional mit der Belastung wachsende Strecke folgt hierauf eine solche, innerhalb welcher dem gleichen Belastungszuwachs ein geringerer Biegungszuwachs entspricht. Dies Verhalten kann bis zum Bruch des Stäbchens unverändert das gleiche bleiben;

1) Seite 59 von: „Elastizität und Festigkeit der Knochen“. 
in anderen Fällen steigen die Biegungszuwüchse gegenüber den Belastungszuwüchsen, in geringerer oder grösserer Entfernung rom Bruch." Kurz gesagt, stellt RAUBER also eine anfängliche, stärkere und sehr unregelmässige und eine darauffolgende regelmässigere, aber geringere Durchbiegung fest und ist geneigt, das Verhalten während der zweiten Biegungsphase „für das normalere zu erklären, das entgegengesetzte Verhalten (während der ersten Phase) aber teils der Schwierigkeit der Messung, teils Unregelmässigkeiten der Stäbchen zuzuschreiben". Die durch RAUBER beobachtete Weise der Durchbiegung ist genau dieselbe, wie sie unser besprochener Knochen S 24 l. zeigt. Die Annahme Raubers, dass die beobachtete Unregelmässigkeit der Durchbiegung während der ersten Periode der Schwierigkeit der Messung und Unregelmässigkeiten der Stäbchen zuzuschreiben sei, kann ich nicht beipflichten; denn ich fand dieselbe Unregelmässigkeit fast bei allen meinen 200 untersuchten Knochen und glaubte sie besser durch die vorstehend geschilderte ungleichzeitige Beanspruchung der einzelnen Knochenelemente zu erklären.

Das führt zu der Frage, welche Folgen, oder wenn man will, welchen Zweck diese Art der Beanspruchung für die praktische Leistungsfähigkeit des Knochens hätte.

Wenn alle Elemente des Knochens gleichzeitig wirken, werden sie natürlich bei grösseren Anforderungen п. U. auch alle beschädigt; der ganze Knochen ist geschwächt. Bei teilweiser Inanspruchnahme können nur die beanspruchten Teile des Knochens leiden. Der Knochen bleibt also wenigstens teilweise funktionsfähig, er ist nur „angebrochen". Vielleicht ist dies für die Heilung des Knochens das Günstigere. Allerdings würde ein Knochen bei gruppenweiser Wirkung seiner Teile niemals einen so grossen Widerstand leisten können, wie es der Fall sein würde, wenn alle Teile gleichzeitig wirkten.

\section{Torsionsversuche.}

Die Knochen werden häufig auf Torsion beansprucht, aber wohl selten so stark, dass dadurch eine Verletzung der Knochen verursacht werden könnte, denm die Gelenke ermöglichen dann eine Drehung des ganzen Knochens.

Da es nicht ausgeschlossen war, dass Torsionen der Diaphyse die beobachteten Unregelmässigkeiten mit verschuldeten, so wollte ich versuchen, durch einige Experimente dafür einen Anhalt zu gewinnen. Bei der Anbringung der Seitenstifte zur Befestigung des zuerst gebrauchten Messapparates für die Durchbiegung war nicht darauf geachtet worden, auf welcher Seite des Metacarpus, der lateralen oder medialen, die Stifte zu stehen kämen. Nun war es nicht ausgeschlossen, dass die event. Torsion des Knochens nach einer bestimmten Seite hin erfolgte, ständig oder doch in der Hauptsache.

Zur Klarstellung dieser Frage fügte ich noch Torsionsversuche mit 5 Paaren Metacarpalien an. Es standen mir dazu nur Schrittpferde, ebenfalls Schlachttiere, zur Verfügung. Da es hier ja nur auf einen Vergleich 
von links und rechts und nicht auf eine Gegenüberstellung der Rassen ankam, konnte ich auf Knochen von Laufpferden hierbei auch verzichten.

Die Versuche wurden folgendermassen ausgeführt: Beide Enden des Knochens wurden in kräftigen, eisernen Kasten von etwa $9 \mathrm{~cm}$ lichter Weite fest eingegipst. Der Kasten mit dem unteren distalen Ende war feststehend, der für das proximale Ende aber war in der Richtung der Längsachse des Knochens mit einer kräftigen eisernen Welle verbunden, welche am andern Ende ein Rad von $50 \mathrm{~cm}$ Radius trug. Die ganze Vorrichtung war horizontal auf einem schweren Gestell befestigt. An einer Seite des Rades war eine Schale für die Aufnahme von Gewichten an einem am Radkranz befestigten Eisenband aufgehängt. Am Rade war noch eine Skala angebracht, um die Grösse der Torsion nachzuweisen. Fig. 34 zeigt die Vorrichtung.

Da das distale Ende immer im feststehenden, das proximale immer im drehbaren Kasten lag und das Rad immer nach derselben Seite, nach rechts, gedreht wurde, so wurden auch die beiden Knochen eines Paares immer nach rechts tordiert, das war aber für den rechten Metacarpus nach aussen, für den linken nach innen. Es konnte sich hierbei nun zeigen, ob je nach der Torsionsrichtung die Knochen eine verschiedene Festigkeit hatten.

Die Schale für die Ge-

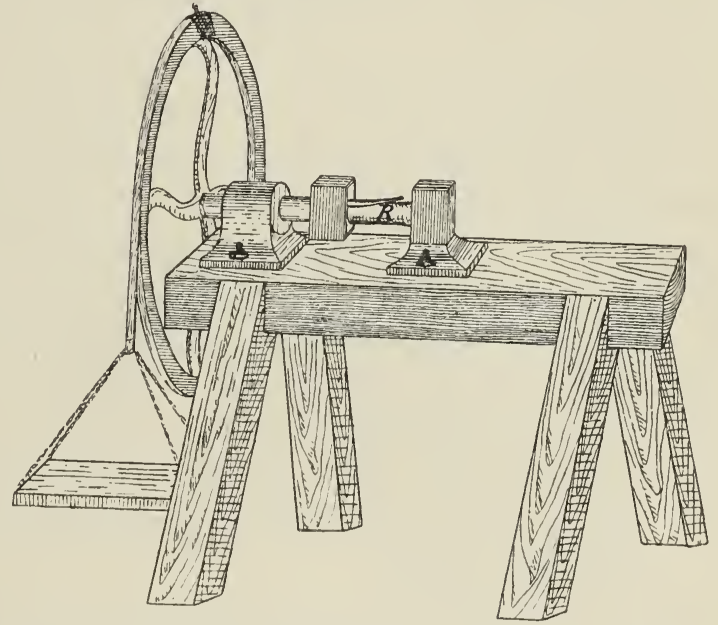

Fig. 34. ca. $1 / 20$ nat. Grösse. Apparat zu den Torsionsversuchen. $R$ das in zwei Eisenkästen eingegipste Röhrbein. Der rechte Eisenkasten auf dem Gestell fest verschraubt, der linke mittelst der Radwelle drehbar. An der Peripherie des Rades ein Stahlband befestigt, welches die Schale zur Aufnahme der Gewichte trägt. wichte wurde gleich anfangs mit $50 \mathrm{~kg}$ belastet und von hier ab die Drehung bei je $5 \mathrm{~kg}$ Mehrbelastung festgestellt. Die Knochen wurden etwa $7 \mathrm{~cm}$ tief eingegipst, und zwar die Knochen jedes Paares immer möglichst gleich weit.

Tabelle XIV zeigt die gefundenen Zahlen, denen ich auch die Knochenmalse beigefügt habe. Die Knochen sind, um eine Verwechselung mit den zu Biegeversuchen benutzten vorzubeugen, nicht mit Nummern, sondern mit Buchstaben bezeichnet.

Mit Ausnahme von No. D ertrug immer der rechte Metacarpus, das ist der im Kniegelenk nach aussen gedrehte, eine etwas grössere Belastung. Die Differenz ist aber nur bei C eine genügend grosse, um daraus auf eine faktisch grössere Torsionsfestigkeit bei nach aussen gerichteter Drehung schliessen zu können. Da aber die Biegeversuche zeigten, dass häufig ein erheblicher Unterschied in der Leistungsfähigkeit der beiden Knochen eines 
Paares vorhanden ist, muss es doch zweifelhaft erscheinen, ob der grössere Torsionswiderstand ganz oder auch nur teilweise durch die Torsionsrichtung verursacht ist und nicht vielmehr seinen Grund in einer verschiedenen Widerstandskraft beider Knochen des betr. Paares hat. Auch das entgegengesetzte Verhalten der Knochen von D spricht dagegen. Auffallend ist ferner die Differenz in der Grösse der Torsion zwischen D l. und r., welche der angewandten Kraft gerade entgegengesetzt ist. Ich wage deshalb nicht, einen Unterschied in der Torsionsfestigkeit je nach der Torsionsrichtung anzunehmen.

Auffallend war die Form des Torsionsbruches: steil aufsteigende, sich teilende Schraubenlinien in der Richtung der Torsion.

\section{Zusammenfassung der Resultate.}

\section{A. Die Form betreffend.}

1. Da die Schrittpferde im Durchschnitt grösser und massiger sind als die Laufpferde, so übertreffen die absoluten Malse der Schrittpferdemetacarpalien sämtlich die der Laufpferde, und zwar ist das absolute Gewicht grösser um $30,7 \%$, das Volumen um $23,7 \%$, die Länge um $3 \%$, der mittlere Durchmesser um $12,4 \%$, der Flächeninhalt des totalen Querschnittes um 29,9\% und die Wandstärke um $4 \%$.

2. Der prozentuale Anteil der Wandstärke am mittleren Querschnittradius ist bei den Schrittpferden um fast $10 \%$ geringer und daher auch das spez. Gewicht des gesamten, unverletzten Metacarpus, nämlich um $1,2 \%$. Das spez. Gewicht der eigentlichen Knochensubstanz ist wahrscheinlich gleich.

3. Im Verhältnis zur Widerristhöhe ist der Metacarpus der Schrittpferde, der grösseren Kurzbeinigkeit entsprechend, um 2,6\% kürzer als bei den Laufpferden.

4. Der Metacarpus der Schrittpferde ist gedrungener gebaut, sein mittlerer Querschnittdurchmesser ist im Verhältnis zur Länge des Metacarpus um $11 \%$ grösser als der der Laufpferde.

5. Der Metacarpus der Schrittpferde ist etwas flacher gebaut als der der Laufpferde.

6. Bei den Laufpferden ist in der Regel die dorsale Knochenwand stärker als die laterale; bei den Schrittpferden ist es meist umgekehrt.

7. Anscheinend sind die Griffelbeine der Schrittpferde (auch relativ) stärker entwickelt als bei den Laufpferden; extreme Stärke der Griffelbeine zeigten nur die Schrittpferde, extreme Rückbildung nur die Laufpferde.

8. Ausserhalb des auf dem Querschnitt errichtet gedachten Zylinders fallen bei beiden Rassen gleiche Anteile der Masse des Metacarpus.

9. Sämtliche relativen Mafse der Knochen zeigen eine so grosse Variabilität, dass die Rassenunterschiede daneben gänzlich verschwinden. 
10. Die Form des Querschnittringes (die Verteilung der Knochenmasse um die Markhöhle herum) ist individuell. Sie wird sehr durch die Griffelbeine beeinflusst.

11. Die Knochenstärke ist im Verhältnis zum Röhrbeinumfang bei den Laufpferden um 1,3\% grösser als bei den Schrittpferden.

12. Der Röhrbeinumfang steht in einem ziemlich konstanten Verhältnis zur Knochenstärke.

\section{B. Die Leistung betreffend.}

1. Die absolute Tragkraft (Biegefestigkeit) der Schrittpferdemetacarpalien ist um $31,7 \%$ grösser als die der Laufpferde.

2. Die Schrittpferdeknochen zeigen auch relativ eine etwas grössere Tragkraft, sowohl auf die Querschnitt- als auch auf die Volumeneinheit berechnet.

3. Die Durchbiegung der Schrittpferderöhren ist absolut eine geringere als bei den Laufpferden.

4. Die Leistungsfähigkeit der Knochensubstanz erscheint bei den Schrittpferden etwas grösser als bei den Laufpferden.

5. Das Verhalten der Knochen während der Biegeversuche weicht stark ab von dem gewöhnlichen Verhalten homogener Körper. 
Tabelle I (Haupttabelle).

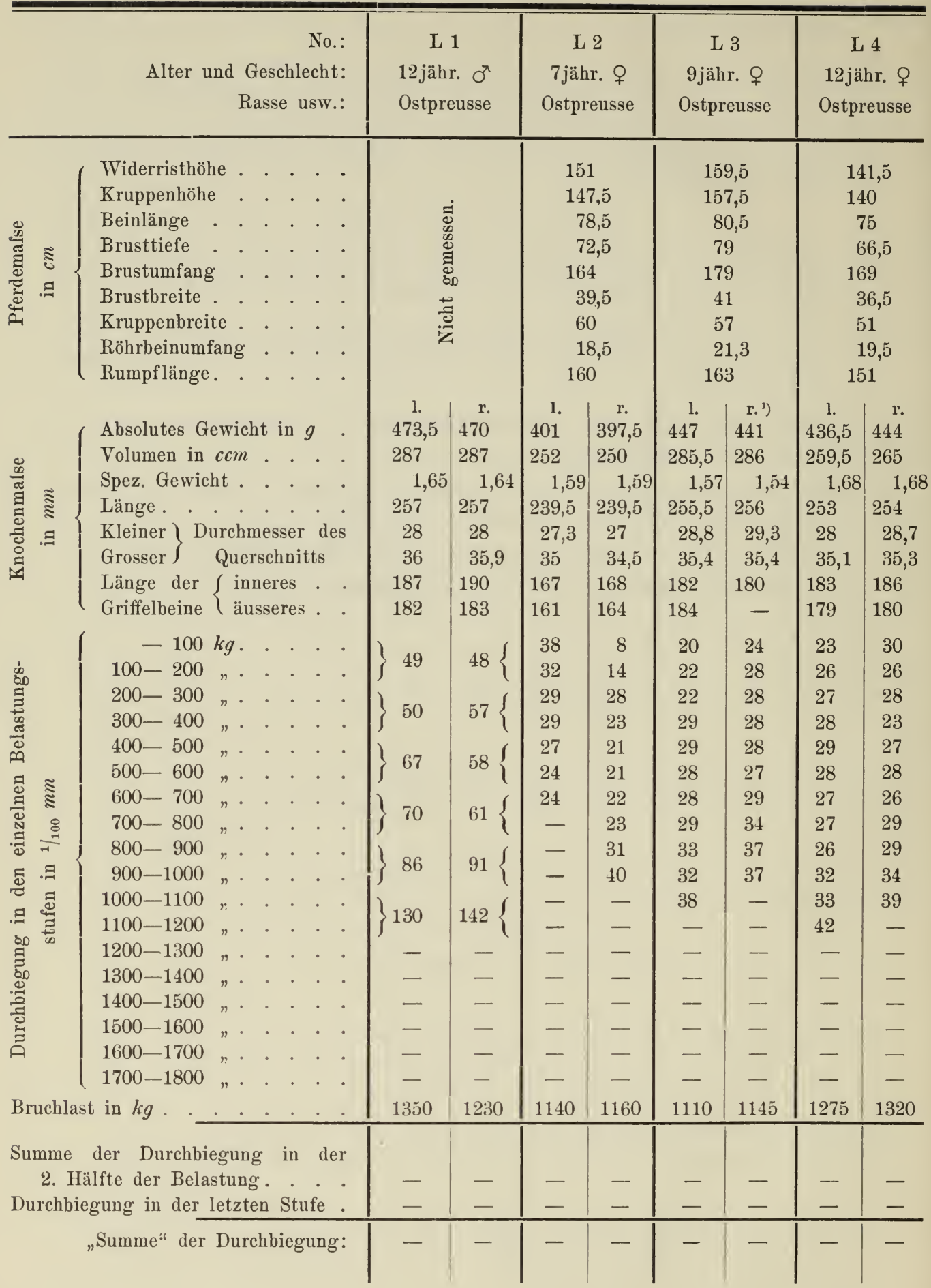

$\left.{ }^{2}\right)$ Ende des äusseren Griffelbeines weggebrochen. 
Tabelle I (Haupttabelle).

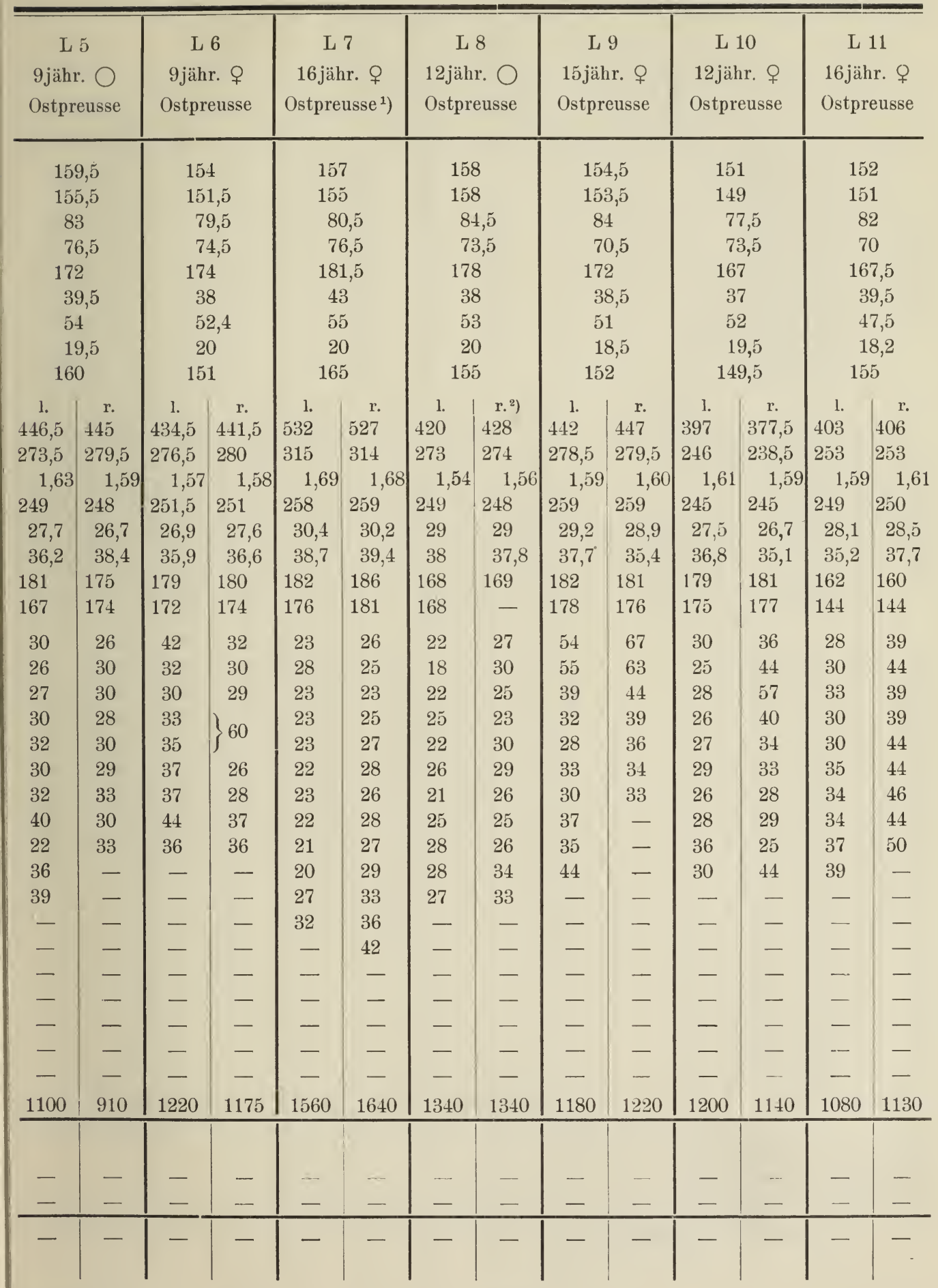

1) Von den 1. Garde-Dragonern. - 2) Spitze war weggebrochen. 
Noch Tabelle I (Haupttabelle).

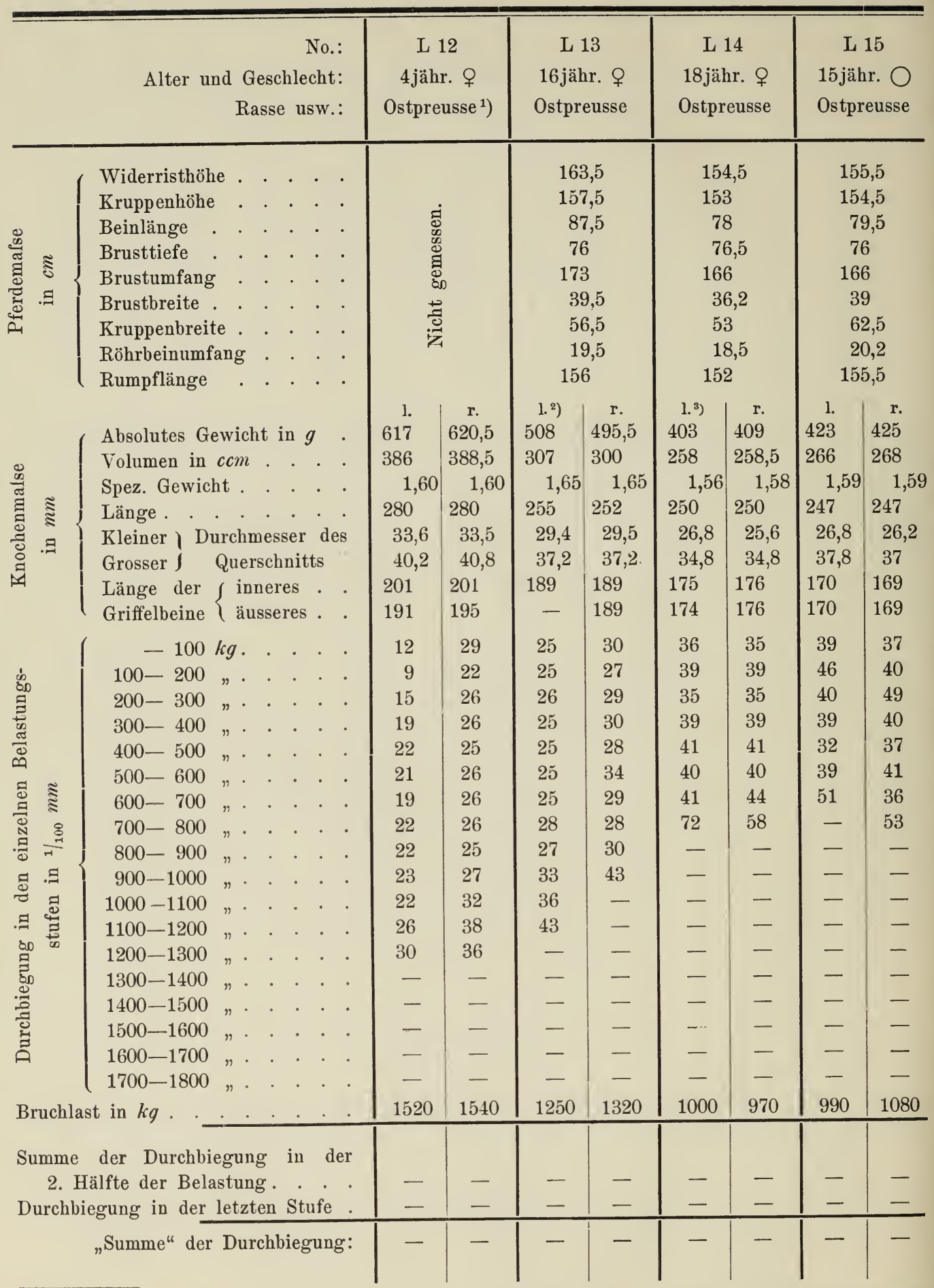

1) Artilleriepferd. - $\left.{ }^{2}\right)$ Äusseres Griffelbein weggebrochen. $-{ }^{3}$ ) Spitze des äusseren Griffelbeins weggebrochen. 
Noch Tabelle I (Haupttabelle).

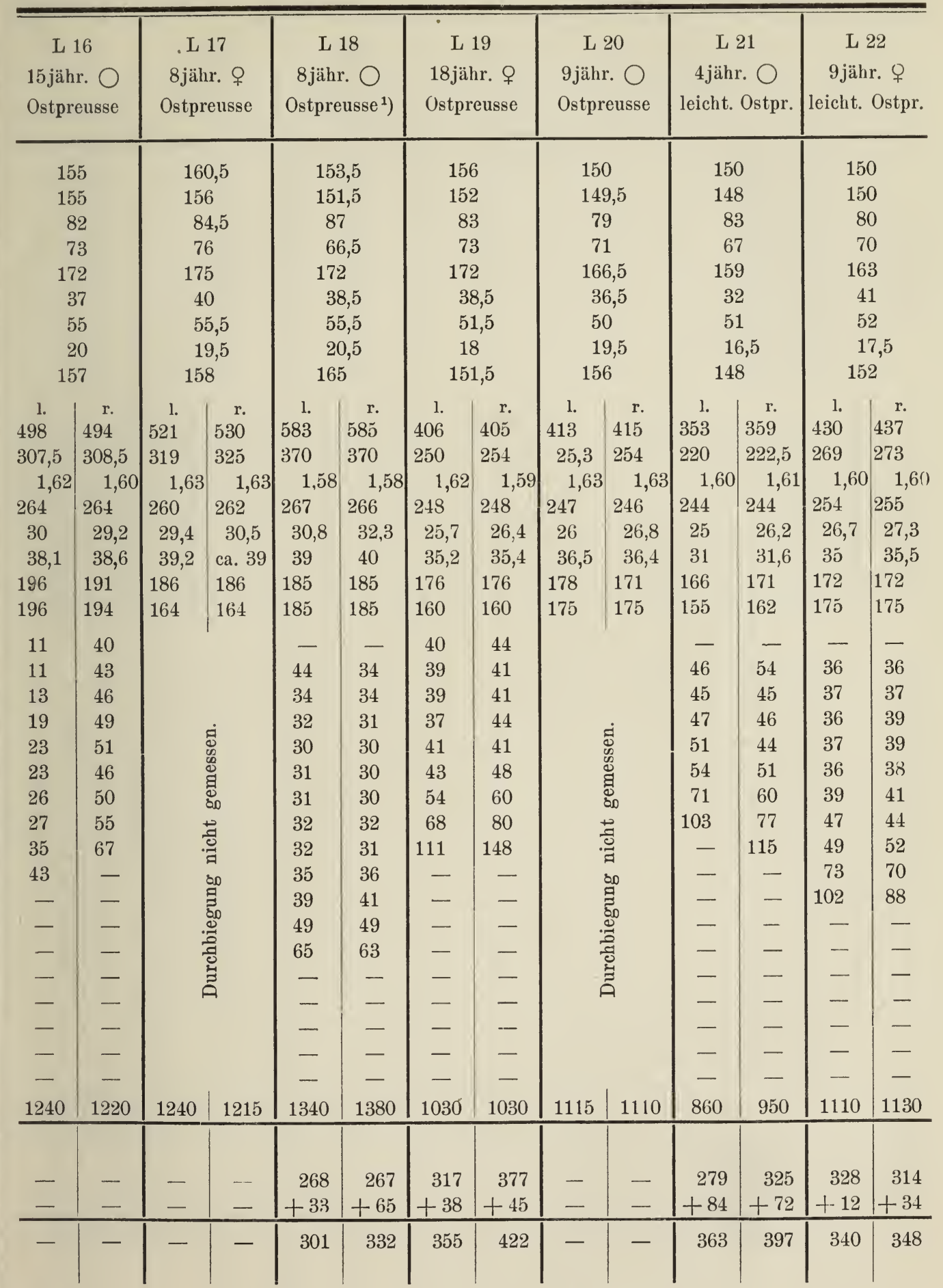

1) Mit Brand: 屯. 
Noch Tabelle I (Haupttabelle).

\begin{tabular}{|c|c|c|c|c|c|c|c|c|c|}
\hline \multicolumn{2}{|c|}{$\begin{array}{r}\text { No.: } \\
\text { Alter und Geschlecht: } \\
\text { Rasse usw.: }\end{array}$} & \multicolumn{2}{|c|}{$\begin{array}{c}\text { L } 23 \\
\text { 5jähr. ㅇ } \\
\text { Ostpreusse }\end{array}$} & \multicolumn{2}{|c|}{ 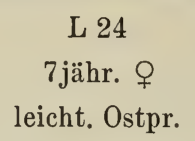 } & \multicolumn{2}{|c|}{$\begin{array}{c}\text { L } 25 \\
\text { 12jähr. ㅇ } \\
\text { Ostpreusse }\end{array}$} & \multicolumn{2}{|c|}{$\begin{array}{c}\text { L } 26 \\
\text { 10jähr. } q \\
\text { leicht. Ostpr. }\end{array}$} \\
\hline 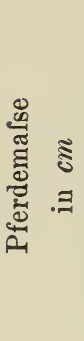 & 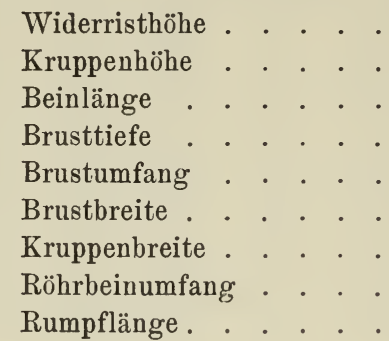 & $\begin{array}{r}16 \\
16 \\
7 \\
8 \\
19 \\
4 \\
5 \\
1 \\
16\end{array}$ & $\begin{array}{l}2 \\
9,5 \\
3,5 \\
3 \\
2 \\
6 \\
9 \\
9\end{array}$ & $\begin{array}{r}15 \\
14 \\
7 \\
7 \\
16 \\
3 \\
5 \\
1 \\
14\end{array}$ & $\begin{array}{l}1 \\
7 \\
6 \\
5 \\
9 \\
6,5 \\
0,5 \\
7,5 \\
6\end{array}$ & $\begin{array}{r}15 \\
15 \\
7 \\
8 \\
17 \\
3 \\
5 \\
1 \\
15\end{array}$ & $\begin{array}{l}6 \\
2 \\
2 \\
4 \\
9 \\
9,5 \\
4,5 \\
8,5 \\
2,5\end{array}$ & $\begin{array}{r}15 \\
15 \\
8 \\
7 \\
16 \\
4 \\
5 \\
1 \\
15\end{array}$ & $\begin{array}{l}51 \\
50 \\
31 \\
30 \\
88 \\
10,5 \\
3,5 \\
8,5 \\
33\end{array}$ \\
\hline 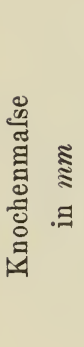 & $\begin{array}{l}\text { Absolutes Gewicht in } g \\
\text { Volumen in } c c m \\
\text { Spez. Gewicht. . . } \\
\text { Länge. } \\
\text { Kleiner } \\
\text { Grosser }\} \text { Durchmesser des } \\
\text { Länge der }\left\{\begin{array}{l}\text { inneres . } \\
\text { Griffelbeine }\end{array} \text { äusseres. }\right.\end{array}$ & $\begin{array}{c}1 . \\
576,5 \\
358,5 \\
1,61 \\
263 \\
31,4 \\
37 \\
193 \\
201\end{array}$ & $\begin{array}{l}\text { r. } \\
572 \\
357 \\
1,60 \\
264 \\
31,5 \\
37,3 \\
194 \\
198\end{array}$ & $\begin{array}{c}1 . \\
388 \\
233,5 \\
1,66 \\
247 \\
25 \\
33,6 \\
169 \\
174\end{array}$ & $\begin{array}{c}\text { r. } \\
390 \\
233,5 \\
1,67 \\
247 \\
26 \\
33,3 \\
165 \\
172\end{array}$ & $\begin{array}{c}1 . \\
445,5 \\
276,5 \\
1,61 \\
246 \\
28,5 \\
37 \\
175 \\
176\end{array}$ & $\begin{array}{c}\mathrm{r} . \\
450,5 \\
279,5 \\
1,61 \\
245 \\
29 \\
37 \\
179 \\
174\end{array}$ & $\begin{array}{c}1 . \\
430 \\
261,5 \\
1,64 \\
243 \\
26,3 \\
35 \\
159 \\
135\end{array}$ & $\begin{array}{c}\mathrm{r} . \\
429 \\
261 \\
1,64 \\
243 \\
26,6 \\
35 \\
161 \\
140\end{array}$ \\
\hline 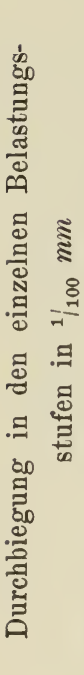 & 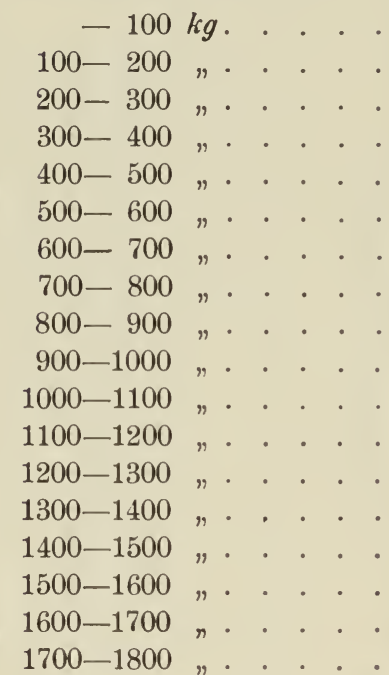 & & & \begin{tabular}{l|}
27 \\
27 \\
25 \\
28 \\
76 \\
41 \\
- \\
- \\
- \\
- \\
- \\
- \\
- \\
- \\
- \\
- \\
- \\
-
\end{tabular} & $\begin{array}{l}23 \\
34 \\
30 \\
34 \\
27 \\
35 \\
55 \\
46 \\
- \\
- \\
- \\
- \\
- \\
- \\
- \\
- \\
-\end{array}$ & & 0 & & 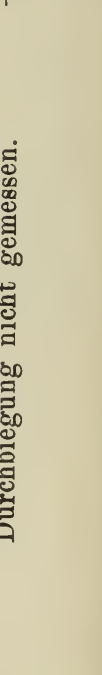 \\
\hline Bruch & $\mathrm{t}$ in $\mathrm{kg} . \ldots . . .$. & 1560 & 1620 & 930 & 1030 & 1380 & 1370 & 1150 & 1170 \\
\hline $\begin{array}{l}\text { Summ } \\
2 . \\
\text { Durch }\end{array}$ & $\begin{array}{l}\text { der Durchbiegung in der } \\
\text { ifte der Belastung. . . . } \\
\text { gung in der letzten Stufe. }\end{array}$ & - & - & - & - & - & - & - & - \\
\hline & "Summe" der Durchbiegung: & - & - & - & - & - & - & - & - \\
\hline
\end{tabular}


Noch Tabelle I (Haupttabelle).

\begin{tabular}{|c|c|c|c|c|c|c|c|c|c|c|c|c|c|}
\hline \multicolumn{2}{|c|}{$\begin{array}{c}\text { L } 27 \\
\text { 5jähr. } \bigcirc \\
\text { Ostpreusse }\end{array}$} & \multicolumn{2}{|c|}{$\begin{array}{c}\text { L } 28 \\
\text { 8jähr. } \bigcirc \\
\text { Ostpreusse }\end{array}$} & \multicolumn{2}{|c|}{$\begin{array}{c}\text { L } 29 \\
5 \text { jähr. } \bigcirc \\
\text { Ostpreusse }\end{array}$} & \multicolumn{2}{|c|}{$\begin{array}{c}\text { L } 30 \\
\text { 9jähr. \& } \\
\text { Ostpreusse }\end{array}$} & \multicolumn{2}{|c|}{$\begin{array}{c}\text { L 31 } \\
\text { 9jähr. ㅇ } \\
\text { Ostpreusse }\end{array}$} & \multicolumn{2}{|c|}{$\begin{array}{c}\text { L } 32 \\
\text { 12jähr. ㅇ } \\
\text { Ostpreusse }\end{array}$} & \multicolumn{2}{|c|}{$\begin{array}{c}\text { L } 33 \\
\text { 14jähr. ㅇ } \\
\text { Ostpreusse }\end{array}$} \\
\hline \multicolumn{2}{|c|}{157} & \multicolumn{2}{|c|}{158} & \multicolumn{2}{|c|}{155,5} & \multicolumn{2}{|c|}{158} & \multicolumn{2}{|c|}{165} & \multicolumn{2}{|c|}{153} & \multicolumn{2}{|c|}{151} \\
\hline \multicolumn{2}{|c|}{153} & \multicolumn{2}{|c|}{154} & \multicolumn{2}{|c|}{152} & \multicolumn{2}{|c|}{159} & \multicolumn{2}{|c|}{160} & \multicolumn{2}{|c|}{151} & \multicolumn{2}{|c|}{149} \\
\hline \multicolumn{2}{|c|}{83,5} & & 1,5 & 8 & & 8 & & 8 & & & 4,5 & & 88,5 \\
\hline & 3,5 & & 6,5 & & 1,5 & 7 & 5 & 7 & & & 8,5 & & 2,5 \\
\hline $17 !$ & & 18 & 5,5 & 16 & & 17 & & 17 & & 16 & & 172 & \\
\hline $3 \varepsilon$ & & & 3,5 & 36 & & & 1,5 & 4 & & & 9,5 & 40 & 0 \\
\hline 5 & & $5 \varepsilon$ & & 4 & & 5 & 2 & & 5,5 & 49 & & & 3,5 \\
\hline & 8,8 & 19 & & & 9,5 & & 7,5 & & 8,8 & & 8,8 & 19 & 9 \\
\hline $15 t$ & & 15 & & 151 & & 16 & & 16 & & 15 & & 15 & 3,5 \\
\hline 1. & $\mathrm{r}$ & 1. & r. & 1. & $r$. & 1. & $r$. & 1. & r. & 1. & r. & 1. & r. \\
\hline 482 & 488 & 505 & 505,5 & 409 & 409 & 442 & 430 & 546 & 526,5 & 443 & 439 & 416 & 422 \\
\hline 300,5 & 303 & 312,5 & 311 & 254 & 254 & 278,5 & 257,5 & 350 & 342,5 & 270 & 272 & 256,5 & 259 \\
\hline 1,61 & 1,61 & 1,62 & 1,62 & 1,61 & 1,61 & 1,59 & 1,67 & 1,56 & 1,57 & 1,64 & 1,61 & 1,62 & 1,63 \\
\hline 254 & 254 & 255 & 256 & 250 & 251 & 257 & 256 & 270 & 269 & 255 & 254 & 253 & 253 \\
\hline 29 & 30 & 27 & 27,3 & 25 & 25 & 27,8 & 28,3 & 30 & 30 & 26,5 & 27,3 & 27,9 & 27,7 \\
\hline 39,4 & 37,6 & 38,3 & 38 & 35,7 & 36 & 35,2 & 36 & 37,4 & 36,5 & 35,4 & ca. 36 & 35,8 & 35,4 \\
\hline 182 & 182 & 178 & 179 & 176 & 178 & 177 & 182 & 195 & 195 & 182 & 178 & 180 & 181 \\
\hline 180 & 175 & 166 & 168 & 175 & 175 & 177 & 181 & 231 & 230 & 179 & 183 & 181 & 180 \\
\hline- & - & - & - & & & - & - & - & - & - & - & - & - \\
\hline 31 & 31 & 33 & 31 & & & 37 & 37 & 41 & 33 & 35 & 37 & 37 & 37 \\
\hline 31 & 34 & 32 & 32 & & & 37 & 37 & 31 & 28 & 34 & 36 & 34 & 33 \\
\hline 31 & 29 & 30 & 30 & & & 35 & 35 & 30 & 31 & 34 & 37 & 33 & 34 \\
\hline 30 & 29 & 30 & 31 & 8 & & 27 & 35 & 31 & 31 & 34 & 39 & 34 & 34 \\
\hline 31 & 31 & 32 & 31 & 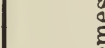 & 象 & 36 & 35 & 29 & 34 & 36 & 40 & 36 & 34 \\
\hline 32 & 31 & 31 & 32 & 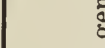 & 20 & 40 & 37 & 33 & 32 & 39 & 43 & 37 & 35 \\
\hline 32 & 31 & 34 & 33 & \pm & & 45 & 40 & 32 & 33 & 42 & 55 & 42 & 39 \\
\hline 37 & 35 & 37 & 36 & e & & 56 & 45 & 35 & 38 & 53 & 72 & 53 & 48 \\
\hline 47 & 43 & 43 & 46 & 8 & 20 & 77 & 57 & 41 & 46 & 64 & - & 69 & 57 \\
\hline 52 & 52 & 53 & 56 & 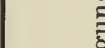 & 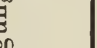 & - & 79 & 52 & 60 & 104 & - & - & 83 \\
\hline 77 & 64 & 74 & 79 & 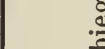 & & - & - & 71 & 96 & - & - & - & - \\
\hline- & - & - & - & ही & & - & - & - & - & - & - & - & - \\
\hline- & - & - & - & 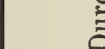 & & - & - & - & - & - & - & - & - \\
\hline- & - & - & - & & & - & - & - & - & - & - & - & - \\
\hline- & - & - & - & & & - & - & - & - & - & - & - & - \\
\hline- & - & - & - & & & - & - & - & - & - & - & - & - \\
\hline- & - & - & - & & & - & - & - & - & - & - & - & - \\
\hline 1200 & 1290 & 1270 & 1230 & 900 & 940 & 1090 & 1190 & 1210 & 1200 & 1140 & 980 & 1040 & 1120 \\
\hline $\begin{array}{l}277 \\
- \\
\end{array}$ & $\begin{array}{r}256 \\
+77 \\
\end{array}$ & $\begin{array}{r}272 \\
+64 \\
\end{array}$ & $\begin{array}{r}282 \\
+28 \\
\end{array}$ & - & - & $\begin{array}{r}254 \\
+92 \\
\end{array}$ & $\begin{array}{r}276 \\
+90 \\
\end{array}$ & $\begin{array}{r}264 \\
+9 \\
\end{array}$ & $\begin{array}{l}305 \\
- \\
\end{array}$ & $\begin{array}{r}320 \\
+45 \\
\end{array}$ & $\begin{array}{r}230 \\
+82 \\
\end{array}$ & $\begin{array}{r}237 \\
+38 \\
\end{array}$ & $\begin{array}{r}279 \\
+20 \\
\end{array}$ \\
\hline 277 & 333 & 336 & 310 & - & - & 346 & 366 & 273 & 305 & 365 & 312 & 275 & 299 \\
\hline
\end{tabular}


Noch Tabelle I (Haupttabelle).

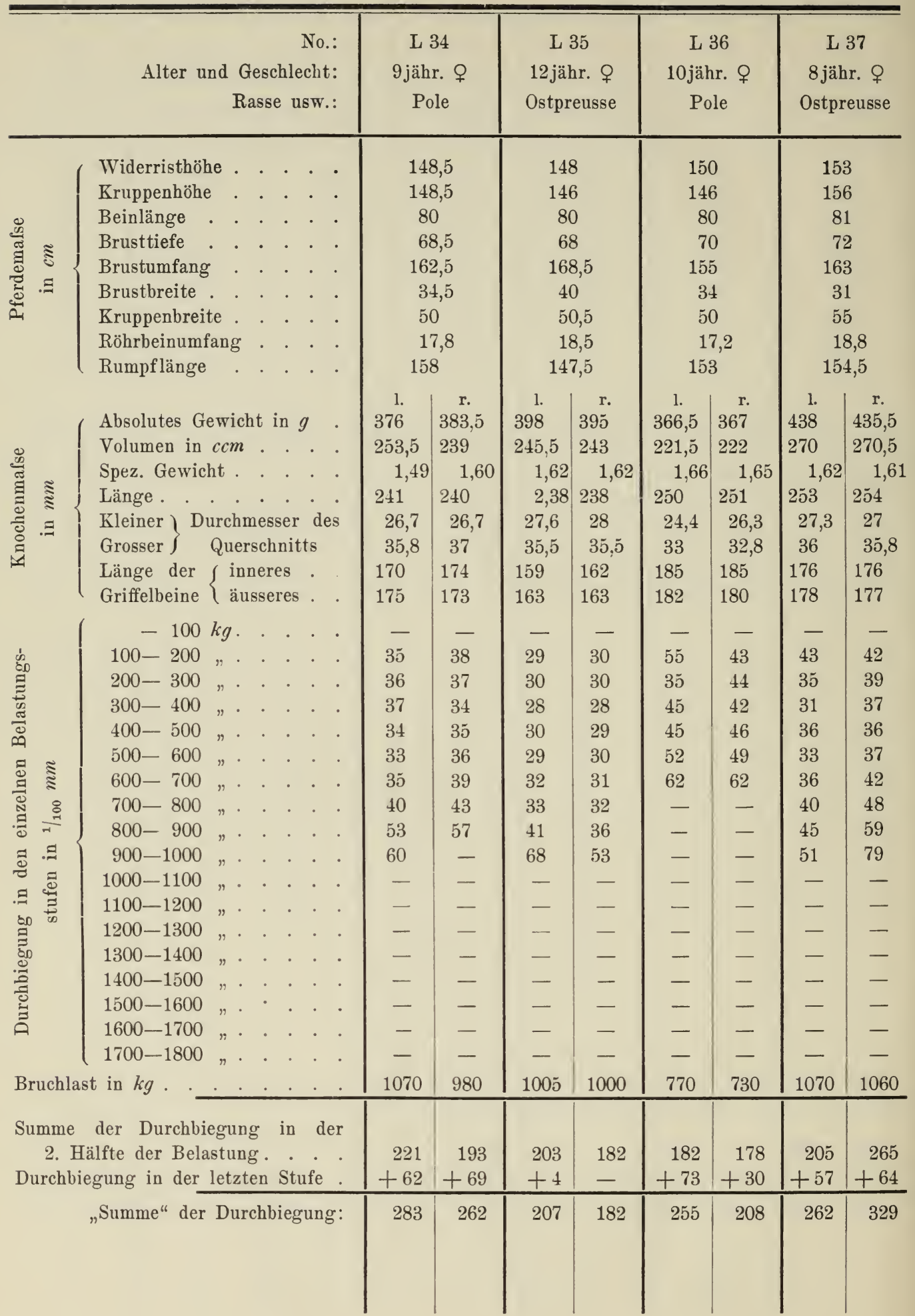


Noch Tabelle I (Haupttabelle).

\begin{tabular}{|c|c|c|c|c|c|c|c|c|c|c|c|c|c|}
\hline \multicolumn{2}{|c|}{$\begin{array}{c}\text { L } 38 \\
12 \text { jähr. 우 } \\
\text { Ostpreusse }\end{array}$} & \multicolumn{2}{|c|}{$\begin{array}{c}\text { L } 39 \\
\text { 15jähr. ㅇ } \\
\text { Ostpreusse }\end{array}$} & \multicolumn{2}{|c|}{$\begin{array}{c}\text { L } 40 \\
\text { 12jähr. } q \\
\text { ? Ostpreusse }\end{array}$} & \multicolumn{2}{|c|}{$\begin{array}{c}\text { L } 41 \\
\text { 15jähr. ㅇ } \\
\text { Ostpreusse }\end{array}$} & \multicolumn{2}{|c|}{$\begin{array}{c}\text { L } 42 \\
\text { 20jähr. }+ \\
\text { leicht. Ostpr. }\end{array}$} & \multicolumn{2}{|c|}{$\begin{array}{c}\text { L } 43 \\
\text { 18jähr. } 9 \\
\text { Ostpreusse }\end{array}$} & \multicolumn{2}{|c|}{$\begin{array}{c}\text { L } 44 \\
\text { 18jähr. } \stackrel{+}{ } \\
\text { Ostpreusse }^{1} \text { ) }\end{array}$} \\
\hline \multicolumn{2}{|c|}{157,5} & \multicolumn{2}{|c|}{154} & \multicolumn{2}{|c|}{155,5} & \multicolumn{2}{|c|}{165} & \multicolumn{2}{|c|}{152} & \multicolumn{2}{|c|}{158} & \multicolumn{2}{|c|}{155,5} \\
\hline \multicolumn{2}{|c|}{156} & \multicolumn{2}{|c|}{151,5} & \multicolumn{2}{|c|}{154,5} & \multicolumn{2}{|c|}{$160, \tilde{5}$} & \multicolumn{2}{|c|}{150} & \multicolumn{2}{|c|}{159} & \multicolumn{2}{|c|}{152} \\
\hline \multirow{2}{*}{\multicolumn{2}{|c|}{$\begin{array}{l}84 \\
73,5\end{array}$}} & 8 & & 79 & & 8 & & 80 & & 83 & 3 & & 82 \\
\hline & & 7 & & & 6,5 & 7 & & 72 & & 75 & 5 & & 73,5 \\
\hline 173 & & 17 & & 180 & & 17 & & 163 & & 183 & & & 67,5 \\
\hline 40 & & 3 & 9,5 & $3 \varepsilon$ & & 4 & & 35 & & 39 & 9 & & 44,5 \\
\hline 51 & & 5 & & 56 & & 5 & & & 1,5 & 58 & 8 & & 52,5 \\
\hline 19 & 9,5 & 19 & & & 9,2 & & 0,3 & 18 & & 20 & 0 & & 18 \\
\hline $15 t$ & 6,5 & 16 & 2,5 & 160 & & 16 & & 15 & 3,5 & 164 & & 15 & 59 \\
\hline 1. & r. & 1. & r. & 1. & r. & 1. & r. & 1. & r. & 1. & r. & I. & $\mathbf{r}$ \\
\hline 486 & 456,5 & 438 & 435,5 & 445 & 424,5 & 546 & 533,5 & 382 & 379,5 & 387,5 & 392,5 & 403 & 416 \\
\hline 297,5 & 294,5 & 283 & 282,5 & 283,5 & $270, \check{5}$ & 346 & 339 & 232,5 & 235,5 & 232,5 & 240 & 266,5 & 262 \\
\hline 1,63 & 1,55 & 1,55 & 1,54 & 1,57 & 1,57 & 1,58 & 1,57 & 1,64 & 1,61 & 1,67 & 1,64 & 1,51 & \begin{tabular}{l|l}
1 & 1,59
\end{tabular} \\
\hline 254 & 257 & 250 & 249 & 243 & 243 & 273 & 274 & 246 & 247 & 242 & 242 & 248 & 248 \\
\hline 28,6 & 28,7 & 28,7 & 28,2 & 27 & 27 & 30 & 29,8 & 26,3 & 25,8 & 26,7 & 26,5 & 28 & 28,7 \\
\hline ca. 38 & 37,5 & 36,8 & 37,2 & 36,4 & 36 & ca. 37 & 37,2 & 33,7 & 32,8 & 35,4 & ca. 35,5 & 36,4 & 37,5 \\
\hline 174 & 176 & 175 & 177 & 180 & 176 & 201 & 196 & 183 & 185 & 171 & 172 & 175 & $175^{\prime}$ \\
\hline 171 & 177 & 174 & 175 & 181 & 173 & 196 & 191 & 177 & 174 & 167 & 170 & 179 & 177 \\
\hline- & - & - & - & - & - & - & - & - & - & - & - & - & - \\
\hline 31 & 38 & 32 & 39 & 46 & 40 & 34 & 35 & 38 & 38 & 34 & 37 & 39 & 38 \\
\hline 28 & 36 & 31 & 37 & 35 & & 33 & 34 & 37 & 36 & 34 & 34 & 35 & 36 \\
\hline 30 & 35 & 30 & 38 & 35 & 3 & 32 & 34 & 37 & 40 & 33 & 34 & 35 & 36 \\
\hline 29 & 37 & 32 & 32 & 33 & 35 & 33 & 33 & 37 & 39 & 32 & 33 & 37 & 36 \\
\hline 29 & 37 & 32 & 35 & 36 & 35 & 33 & 35 & 39 & 39 & 32 & 32 & 39 & 38 \\
\hline 30 & 38 & 35 & 35 & 35 & 36 & 33 & 35 & 42 & 41 & 36 & 36 & 41 & 43 \\
\hline 34 & 47 & 36 & 38 & 38 & 49 & 37 & 37 & 48 & 55 & 38 & 38 & 50 & 50 \\
\hline 36 & 55 & 36 & 40 & 43 & 60 & 41 & 45 & 60 & 65 & 46 & 54 & 72 & 63 \\
\hline 46 & 68 & 40 & 70 & 56 & - & 54 & 55 & 120 & - & 55 & - & - & - \\
\hline- & - & 86 & 81 & - & - & 72 & - & - & - & 81 & - & - & - \\
\hline- & - & 102 & 134 & - & - & - & - & - & - & - & - & - & - \\
\hline- & - & - & - & - & - & - & - & - & - & - & - & - & - \\
\hline- & - & - & - & - & - & - & - & - & - & - & - & - & - \\
\hline- & - & - & - & - & - & - & - & - & - & - & - & - & - \\
\hline- & - & - & - & - & - & - & - & - & - & - & - & - & - \\
\hline- & - & - & - & - & - & - & - & - & - & - & - & - & - \\
\hline- & - & - & - & - & - & - & - & - & - & - & - & - & - \\
\hline 1075 & 1005 & 1255 & 1200 & 1045 & 975 & 1120 & 1095 & 1040 & 990 & 1100 & 975 & 925 & 945 \\
\hline $\begin{array}{r}175 \\
+52 \\
\end{array}$ & $\begin{array}{r}245 \\
+5 \\
\end{array}$ & $\begin{array}{r}335 \\
+61 \\
\end{array}$ & $\begin{array}{l}398 \\
- \\
\end{array}$ & $\begin{array}{r}208 \\
+37 \\
\end{array}$ & $\begin{array}{r}198 \\
+66 \\
\end{array}$ & $\begin{array}{r}254 \\
+18 \\
\end{array}$ & $\begin{array}{r}207 \\
+79 \\
\end{array}$ & $\begin{array}{r}309 \\
+50 \\
\end{array}$ & $\begin{array}{r}220 \\
+88 \\
\end{array}$ & $\begin{array}{l}272 \\
- \\
\end{array}$ & $\begin{array}{r}177 \\
+59 \\
\end{array}$ & $\begin{array}{r}221 \\
+25 \\
\end{array}$ & $\begin{array}{r}212 \\
+38 \\
\end{array}$ \\
\hline 227 & 250 & 396 & 398 & 245 & 264 & 272 & 286 & 359 & 308 & 272 & 236 & 246 & 250 \\
\hline
\end{tabular}

1) Mit Brand: \$ 
Noch Tabelle I (Haupttabelle).

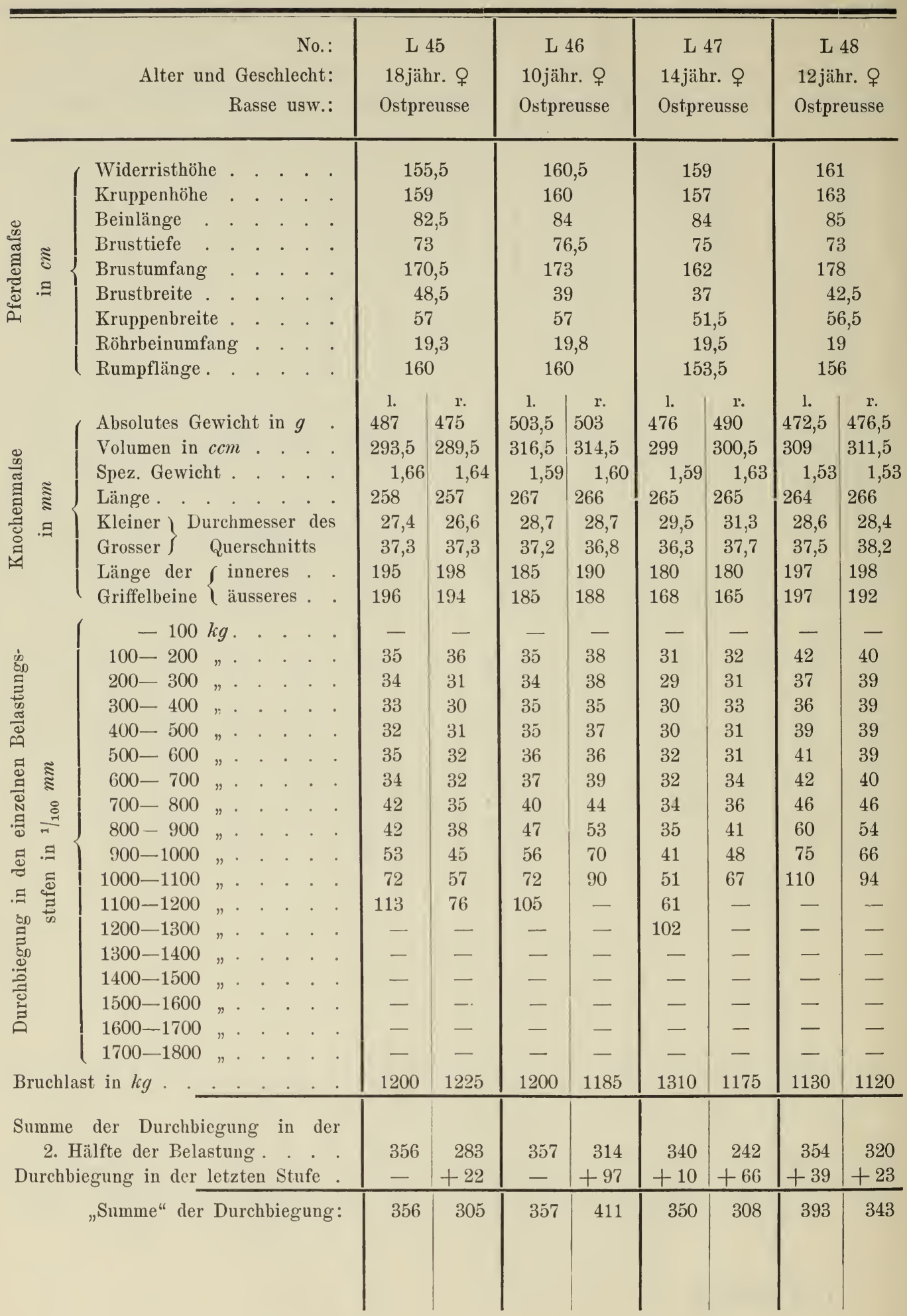


Noch Tabelle I (Haupttabelle).

\begin{tabular}{|c|c|c|c|c|c|c|c|c|c|c|c|c|c|}
\hline \multicolumn{2}{|c|}{$\begin{array}{c}\text { L } 49 \\
\text { 14jähr. } \bigcirc \\
\text { Ostpreusse }^{1} \text { ) }\end{array}$} & \multicolumn{2}{|c|}{$\begin{array}{c}\text { L } 50 \\
\text { 18jähr. }+9 \\
\text { stpreusse }\end{array}$} & \multicolumn{2}{|c|}{$\begin{array}{c}\text { L } 51 \\
\text { 14jähr. ㅇ } \\
\text { Ostpreusse }\end{array}$} & \multicolumn{2}{|c|}{$\begin{array}{c}\text { L } 52 \\
\text { 18jähr. 우 } \\
\text { leicht. Ostpr. }\end{array}$} & \multicolumn{2}{|c|}{$\begin{array}{c}\text { L } 53 \\
\text { 15́jähr. ๆ } \\
\text { leicht. Ostpr. }\end{array}$} & \multicolumn{2}{|c|}{$\begin{array}{c}\text { L } 54 \\
\text { 15jähr. 우 } \\
\text { Ostpreusse }\end{array}$} & \multicolumn{2}{|c|}{$\begin{array}{c}\text { L 555 } \\
\text { 15jähr. O } \\
\text { Ostpreusse }\end{array}$} \\
\hline \multicolumn{2}{|c|}{159,5} & \multicolumn{2}{|c|}{155,5} & \multicolumn{2}{|c|}{159,5} & \multicolumn{2}{|c|}{148,5} & \multicolumn{2}{|c|}{153,5} & \multicolumn{2}{|c|}{154} & \multicolumn{2}{|c|}{152,5} \\
\hline \multicolumn{2}{|c|}{156,5} & \multicolumn{2}{|c|}{150} & \multicolumn{2}{|c|}{154,5} & \multicolumn{2}{|c|}{148,5} & \multicolumn{2}{|c|}{153} & \multicolumn{2}{|c|}{155} & \multicolumn{2}{|c|}{151} \\
\hline 88 & & \multicolumn{2}{|c|}{83,5} & 83 & 3,5 & 79 & & 8 & & 81 & & 81 & \\
\hline 71 & 1,5 & 7 & & 76 & & 69 & & & 1,5 & 73 & & & 1,5 \\
\hline 167 & & 16 & & 175 & & 162 & & 17 & & 185 & & 16 & \\
\hline 37 & 7,5 & 3 & & 37 & 7,5 & 33 & & $3 \varepsilon$ & & 41 & 1,2 & & 0,5 \\
\hline & 2,5 & & 1,5 & 58 & & 52 & & 5 & 1,5 & - & & 50 & 0 \\
\hline & 9,5 & & 3,5 & 19 & 9,5 & 17 & 7,5 & 18 & & 20 & & 19 & \\
\hline 158 & & 14 & & 168 & & 155 & & 15 & 2,5 & 159 & & 14 & \\
\hline 1. & r. & 1. & r. & 1. & $\mathrm{r}$ & 1. & r. & 1. & r. & 1. & r. & & r. \\
\hline 459,5 & 465,5 & 426,5 & 423 & 483,5 & 501,5 & 393 & 381,5 & 423 & 420,5 & 484,5 & 490 & 430,5 & 431,5 \\
\hline 291 & 299 & 264,5 & 262,5 & 307,5 & 329,5 & 249 & 247,5 & 254 & 252,5 & 297 & 303 & 266,5 & 267,5 \\
\hline 1,58 & 1,56 & 1,61 & 1,61 & 1,57 & 1,52 & 1,58 & 1,54 & 1,67 & 1,67 & 1,63 & 1,62 & 1,62 & 1,61 \\
\hline 257,5 & 257 & 252 & 251 & 258 & 258 & 251 & 250 & 258 & 256 & 254 & 254 & 244 & 244 \\
\hline 28,6 & 29 & 28,5 & 28,2 & 27,4 & 27,4 & 27 & 26,7 & 26,8 & 27,4 & 28,6 & ca. 30 & 27,8 & 27,2 \\
\hline 36,8 & 37,8 & 35,4 & 35,8 & 37,4 & 37,5 & 34,6 & 34,4 & 34,2 & 34,2 & 38,7 & 38,3 & 38 & 38,4 \\
\hline 177 & 178 & 172 & 171 & 196 & 196 & 173 & 172 & 185 & 188 & 181 & 185 & 169 & 167 \\
\hline 165 & 167 & 171 & 171 & 192 & 192 & 168 & 156 & 177 & 176 & 178 & 181 & 181 & 185 \\
\hline- & - & - & - & - & - & - & - & - & - & - & - & - & - \\
\hline 32 & 35 & 37 & 32 & 32 & 38 & 41 & 40 & 46 & 46 & 28 & 29 & 29 & 30 \\
\hline 30 & 30 & 31 & 28 & 31 & 35 & 38 & 38 & 40 & 38 & 29 & 26 & 27 & 29 \\
\hline 29 & 33 & 30 & 28 & 33 & 35 & 40 & 36 & 40 & 36 & 28 & 26 & 26 & 30 \\
\hline 30 & 31 & 31 & 29 & 32 & 33 & 38 & 40 & 40 & 39 & 27 & 24 & 26 & 27 \\
\hline 31 & 31 & 31 & 30 & 32 & 34 & 42 & 39 & 39 & 40 & 29 & 26 & 26 & 27 \\
\hline 32 & 33 & 31 & 32 & 33 & 35 & 44 & 44 & 42 & 40 & 26 & 25 & 29 & 27 \\
\hline 35 & 36 & 34 & 35 & 39 & 39 & 55 & 52 & 48 & 37 & 29 & 27 & 27 & 29 \\
\hline 36 & 43 & 41 & 43 & 40 & 43 & 71 & 68 & 56 & 57 & 29 & 28 & 33 & 31 \\
\hline 48 & 50 & 43 & 46 & 52 & 52 & 119 & 110 & 76 & 73 & 34 & 29 & 35 & - \\
\hline 56 & 71 & 55 & 56 & 61 & 69 & - & - & - & - & 37 & 35 & 43 & - \\
\hline 83 & - & 74 & 72 & 96 & 95 & - & - & - & - & 49 & 37 & 57 & - \\
\hline- & - & - & - & - & - & - & - & - & - & - & 47 & - & - \\
\hline- & - & - & - & - & - & - & - & - & - & - & - & - & - \\
\hline- & - & - & - & - & - & - & - & - & - & - & - & - & - \\
\hline- & - & - & - & - & - & - & - & - & - & - & - & - & - \\
\hline- & - & - & - & - & - & - & - & - & - & - & - & - & - \\
\hline- & - & - & - & - & - & - & - & - & - & - & - & - & - \\
\hline 1250 & 1110 & 1230 & 1260 & 1210 & 1220 & 1020 & 1050 & 1080 & 1040 & 1260 & 1380 & 1230 & 950 \\
\hline $\begin{array}{r}290 \\
+48 \\
\end{array}$ & $\begin{array}{r}249 \\
+9 \\
\end{array}$ & $\begin{array}{r}278 \\
+28 \\
\end{array}$ & $\begin{array}{r}275 \\
+55 \\
\end{array}$ & $\begin{array}{r}321 \\
+11 \\
\end{array}$ & $\begin{array}{r}333 \\
+22 \\
\end{array}$ & $\begin{array}{r}331 \\
+26 \\
\end{array}$ & $\begin{array}{r}313 \\
+63 \\
\end{array}$ & $\begin{array}{r}261 \\
+84 \\
\end{array}$ & $\begin{array}{r}247 \\
+40 \\
\end{array}$ & $\begin{array}{r}204 \\
+41 \\
\end{array}$ & $\begin{array}{r}216 \\
+53 \\
\end{array}$ & $\begin{array}{r}224 \\
+22 \\
\end{array}$ & $\begin{array}{r}128 \\
+28 \\
\end{array}$ \\
\hline 338 & 258 & 306 & 330 & 332 & 355 & 357 & 376 & 345 & 287 & 245 & 269 & 246 & 156 \\
\hline
\end{tabular}

1) Von den 1. Garde-Dragonern. 
Noch Tabelle I (Haupttabelle).

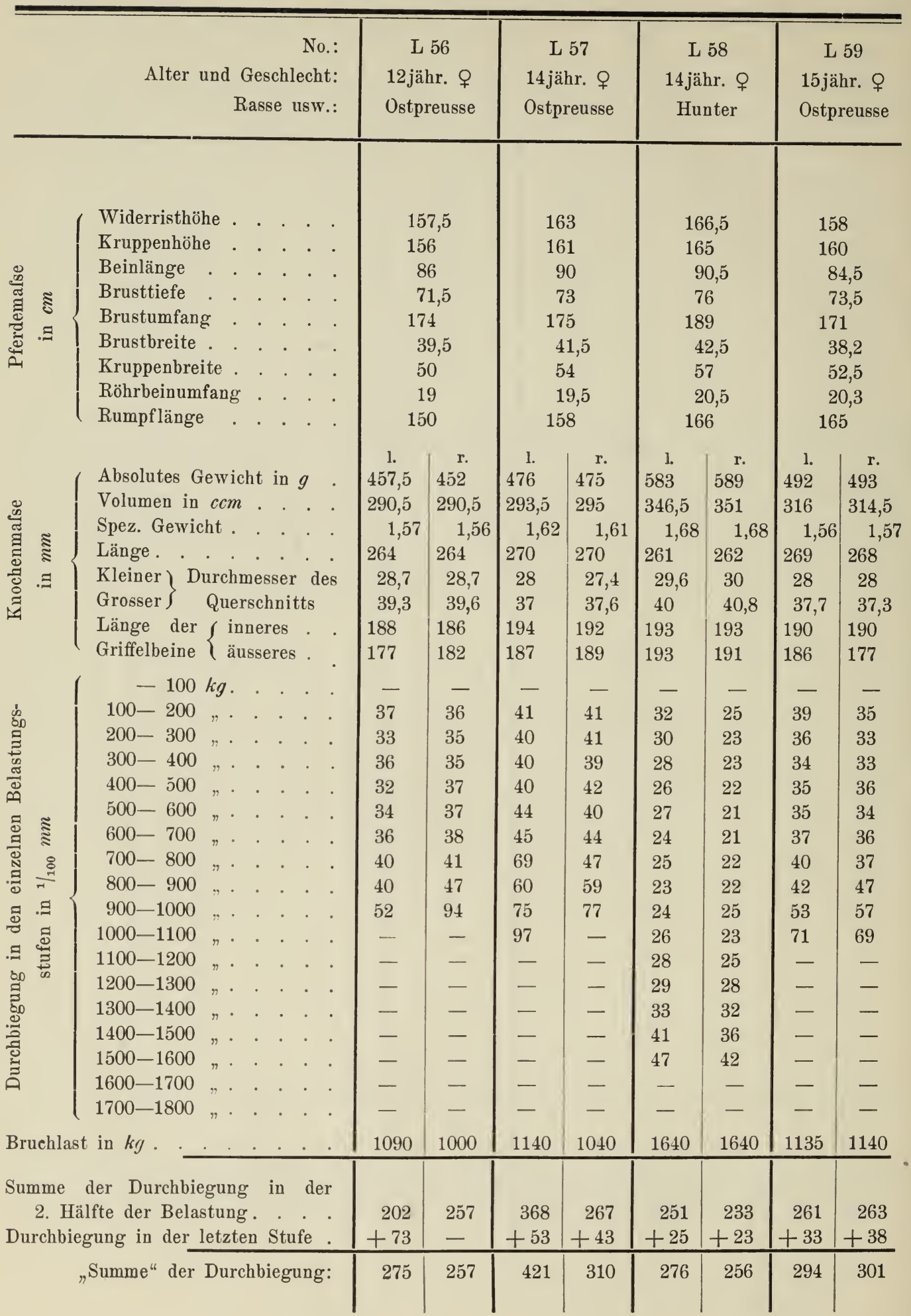


Noch Tabelle I (Haupttabelle).

\begin{tabular}{|c|c|c|c|c|c|c|c|c|c|c|c|c|c|}
\hline \multicolumn{2}{|c|}{$\begin{array}{c}\text { L } 60 \\
\text { 1jähr. } \bigcirc \\
\text { Laufpferd }\end{array}$} & \multicolumn{2}{|c|}{$\begin{array}{c}\text { L } 61 \\
12 \text { jähr. } q \\
\text { Jucker }\end{array}$} & \multicolumn{2}{|c|}{$\begin{array}{c}\text { S } 1 \\
\text { 15jähr. } \bigcirc \\
\text { Däne }\end{array}$} & \multicolumn{2}{|c|}{$\begin{array}{c}\text { S } 2 \\
\text { 6jähr. 우 } \\
\text { Belgier }\end{array}$} & \multicolumn{2}{|c|}{$\begin{array}{c}\text { S } 3 \\
\text { 12jähr. ㅇ } \\
\text { Belgier }\end{array}$} & \multicolumn{2}{|c|}{$\begin{array}{c}\text { S } 4 \\
\text { 10jähr. } \bigcirc \\
\text { Belgier }\end{array}$} & \multicolumn{2}{|c|}{$\begin{array}{c}\text { S 5 } \\
\text { 16jähr. } \bigcirc \\
\text { Belgier }\end{array}$} \\
\hline Absolut & $\begin{array}{l}\% \% \text { der } \\
\text { widerrist- } \\
\text { höhe }\end{array}$ & Absolut & $\begin{array}{c}\% \text { der } \\
\text { Widerrist- } \\
\text { höhe }\end{array}$ & & & & & & & & & & \\
\hline 46,5 & 100 & 133 & 100 & 160 & & 152 & & 164 & & 16 & 9,5 & 161 & 1,5 \\
\hline 141,5 & 96,6 & 129 & 97,4 & 159 & & 154 & & 166 & & 168 & & 159 & 9,5 \\
\hline 85,5 & 58,4 & 73 & 54,9 & & 2,5 & 74 & & 81 & 1,5 & 8 & & 84 & 4 \\
\hline 61 & 41,6 & 60 & 45,1 & & 7,5 & & 8,5 & 83 & & & 4,5 & & 7,5 \\
\hline 144 & 98,4 & 164 & 123,3 & 192 & & 204 & & 189 & & 20 & & 198 & \\
\hline 29,7 & 20,2 & 36 & 27,1 & 43 & & 48 & & 44 & & & 9,5 & & 5,5 \\
\hline 39 & 26,6 & 48 & 36,7 & 54 & & & 4,5 & 6 & & & 8,5 & 63 & 3 \\
\hline 15,5 & 10,6 & 18,5 & 13,9 & & 1,5 & 21 & & & 4,5 & & 6,5 & & 1,5 \\
\hline 138 & 94,2 & 146 & 109,8 & 162 & & 146 & & 165 & & 15 & 9,5 & 157 & \\
\hline 286 & $\begin{array}{r}\text { r. } \\
270\end{array}$ & $\begin{array}{r}1 . \\
356\end{array}$ & $\begin{array}{c}\text { r. } \\
364,5\end{array}$ & $\begin{array}{r}1 . \\
472\end{array}$ & $\begin{array}{r}\text { r. } \\
466\end{array}$ & $\begin{array}{c}1 . \\
612,5\end{array}$ & $\begin{array}{r}\mathrm{r} . \\
606\end{array}$ & $\begin{array}{c}1 . \\
709,5\end{array}$ & $\begin{array}{r}\text { r. } \\
690\end{array}$ & $\begin{array}{r}1 . \\
697\end{array}$ & $\begin{array}{r}\text { r. } \\
699\end{array}$ & $\begin{array}{c}1 . \\
568,5\end{array}$ & $\mid \begin{array}{r}\text { r. } \\
570\end{array}$ \\
\hline 208 & 203 & 219 & 223,5 & 300 & 294,5 & 374,5 & 371 & 450 & 457 & 446 & 445,5 & 355 & 354,5 \\
\hline 1,37 & 1,33 & 1,63 & 1,63 & 1,57 & 1,58 & 1,64 & 1,63 & 1,58 & 1,60 & 1,56 & 1,55 & 1,60 & 1,61 \\
\hline 258 & 256 & 239 & 240 & 258 & 259 & 250,5 & 252,5 & 268 & 268 & 267 & 268 & 258 & 260 \\
\hline 23 & 22,5 & 26,1 & 26,3 & 27,9 & 27,2 & 31,9 & 31,5 & 32,1 & 31,9 & 33,7 & 34,7 & 30,8 & 31,7 \\
\hline 29,6 & 29,5 & 34,2 & 34,4 & 36,5 & 36,6 & 43,8 & 42,3 & 44,8 & 44,1 & 49,6 & 47,1 & 42,6 & 42,3 \\
\hline 186 & 187 & 165 & 165 & 178 & 178 & 172 & 178 & 192 & 193 & 188 & 188 & 178 & 180 \\
\hline 185 & 184 & 167 & 167 & 167 & 167 & 185 & 188 & 199 & 199 & 189 & 189 & 175 & 179 \\
\hline 46 & 100 & 29 & 29 & & & 17 & 10 & 25 & 44 & 20 & 37 & 26 & 30 \\
\hline 63 & 106 & 37 & 34 & & $43<$ & 14 & 10 & 27 & 40 & 22 & 43 & 23 & 35 \\
\hline 69 & 113 & 36 & 36 & ${ }_{5}$ & & 17 & 11 & 26 & 30 & 21 & 34 & 23 & 22 \\
\hline 75 & 131 & 38 & 36 & $\int 50$ & $50\}$ & 16 & 20 & 25 & 32 & 21 & 28 & 21 & 26 \\
\hline 85 & 172 & 36 & 34 & )$_{53}$ & 57 & 18 & 26 & 21 & 33 & 18 & 27 & 21 & 22 \\
\hline- & - & 42 & 41 & 53 & $57<$ & 18 & 11 & 15 & 30 & 15 & 27 & 18 & 20 \\
\hline- & - & 43 & 41 & )$_{58}$ & $61 \mathrm{f}$ & 17 & 18 & 15 & 30 & 19 & 25 & 23 & 19 \\
\hline- & - & 49 & 51 & jo & $61\}$ & 21 & 17 & 18 & 35 & 15 & 25 & 22 & 21 \\
\hline- & - & - & - & $I_{72}$ & & 18 & 11 & 14 & 34 & 11 & 25 & 25 & 21 \\
\hline- & - & - & - & \}$^{73}$ & 64 & 19 & 16 & 13 & 28 & 13 & 21 & 25 & 21 \\
\hline- & - & - & - & $\lambda_{79}$ & - & 19 & 21 & 13 & 33 & 14 & 21 & 23 & 22 \\
\hline- & - & - & - & $7^{72}$ & - & 17 & 19 & 18 & 32 & 13 & 22 & 30 & 25 \\
\hline- & - & - & - & - & - & 19 & 18 & 19 & 29 & 13 & 23 & 32 & 25 \\
\hline- & - & - & - & - & - & 18 & 28 & 18 & 29 & 15 & 27 & - & 32 \\
\hline- & - & - & 一 & - & - & 26 & 20 & - & - & 15 & - & - & - \\
\hline- & - & - & - & - & - & - & - & - & - & - & - & - & - \\
\hline- & - & - & - & - & - & - & - & - & - & - & - & - & - \\
\hline- & - & - & - & - & - & - & - & - & - & - & - & - & - \\
\hline 595 & 540 & 825 & 945 & 1200 & 1080 & 1850 & 1770 & 1630 & 1400 & 1680 & 1790 & 1600 & 1540 \\
\hline- & - & - & - & - & - & - & - & - & - & - & - & - & - \\
\hline- & - & - & - & - & - & - & - & - & - & - & - & - & - \\
\hline- & - & - & - & - & - & - & - & - & - & - & - & - & - \\
\hline
\end{tabular}


Noch Tabelle I (Haupttabelle).

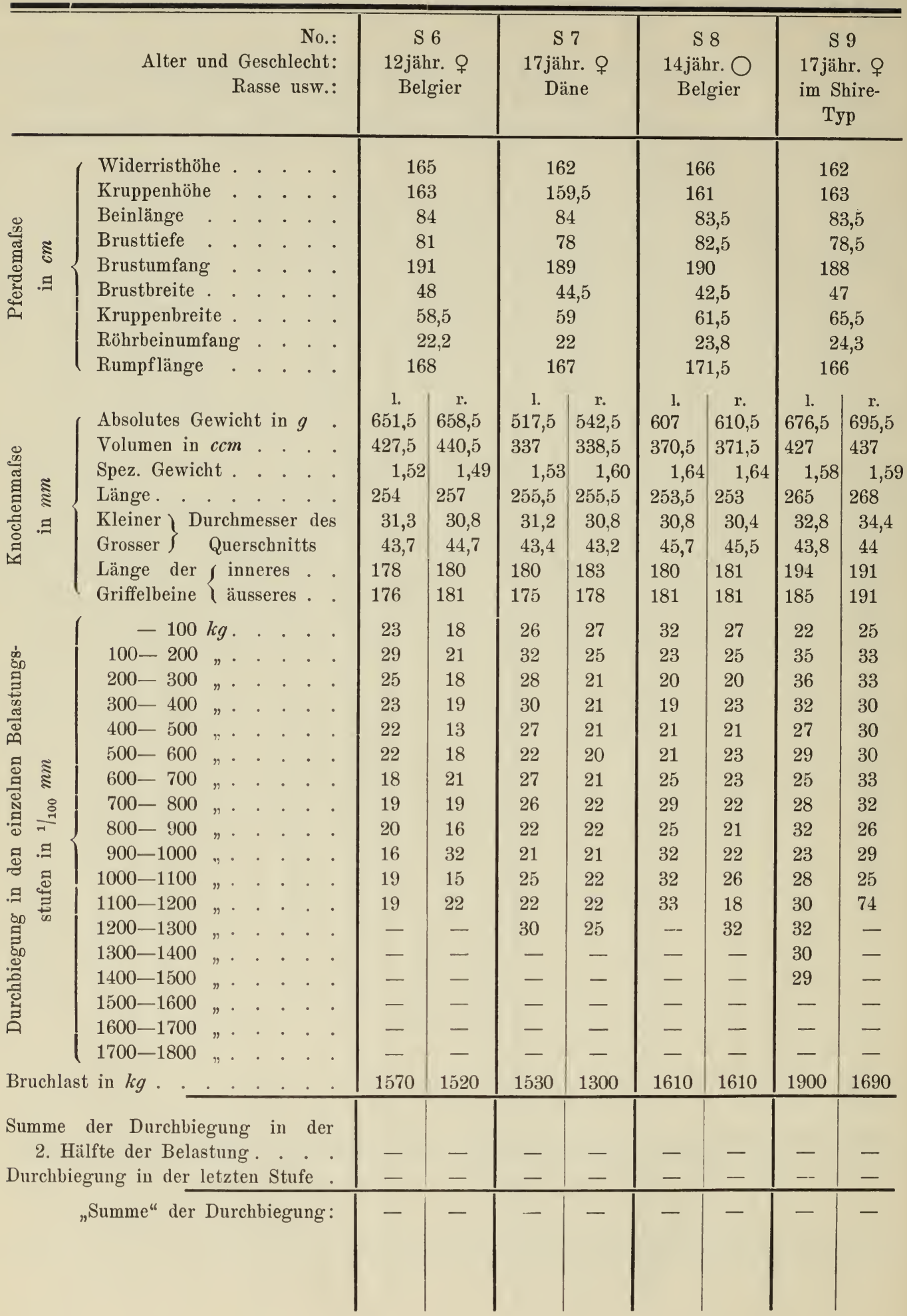


Noch Tabelle I (Haupttabelle).

\begin{tabular}{|c|c|c|c|c|c|c|c|c|c|c|c|c|c|}
\hline \multicolumn{2}{|c|}{$\begin{array}{c}\text { S } 10 \\
\text { 12jähr. } \bigcirc \\
\text { Däne }\end{array}$} & \multicolumn{2}{|c|}{$\begin{array}{c}\text { S } 11 \\
\text { 12jähr. 우 } \\
\text { im Shire- } \\
\text { Typ }\end{array}$} & \multicolumn{2}{|c|}{$\begin{array}{c}\text { S } 12 \\
\text { 16jähr. ㅇ } \\
\text { Belgier }\end{array}$} & \multicolumn{2}{|c|}{$\begin{array}{c}\text { S } 13 \\
14 \text { jähr. } \bigcirc \\
\text { Belgier }\end{array}$} & \multicolumn{2}{|c|}{$\begin{array}{c}\text { S } 14 \\
\text { 15jähr. } \bigcirc \\
\text { Belgier }\end{array}$} & \multicolumn{2}{|c|}{$\begin{array}{c}\text { S } 15 \\
\text { 14 jähr. } \bigcirc \\
\text { Belgier }\end{array}$} & \multicolumn{2}{|c|}{$\begin{array}{c}\text { S } 16 \\
\text { 15jähr. } \bigcirc \\
\text { Belgier }\end{array}$} \\
\hline \multicolumn{2}{|c|}{161} & \multicolumn{2}{|c|}{164,5} & \multicolumn{2}{|c|}{154} & \multicolumn{2}{|c|}{169} & \multicolumn{2}{|c|}{161} & \multicolumn{2}{|c|}{165} & \multicolumn{2}{|c|}{158} \\
\hline \multicolumn{2}{|c|}{158} & \multicolumn{2}{|c|}{165,5} & \multicolumn{2}{|c|}{154,5} & \multicolumn{2}{|c|}{169} & \multicolumn{2}{|c|}{164} & \multicolumn{2}{|c|}{167,5} & \multicolumn{2}{|c|}{159} \\
\hline \multirow{2}{*}{\multicolumn{2}{|c|}{$\begin{array}{l}82,5 \\
78,5\end{array}$}} & & 4,5 & $7 \varepsilon$ & 8,5 & 86 & & 84 & & 83 & & & 77 \\
\hline & & & 9,7 & 75 & 5,5 & 83 & & 77 & & 82 & & & 31 \\
\hline 190 & & 186 & 6,5 & 186 & 6,5 & 197 & & 201 & & 194 & & 19 & 97 \\
\hline 43 & & & 1,5 & 4 & 3,5 & 46 & 6,5 & 48 & & & 3,5 & & 42 \\
\hline 58 & & & 9,5 & 57 & 7,5 & 67 & & 63 & & 65 & & & 62 \\
\hline 22 & 2,5 & & 3,2 & 2 & & & 7,5 & 22 & & & 3,5 & & 22,5 \\
\hline 170 & & 172 & 2,5 & $15:$ & 9,5 & 174 & & 172 & & 16 & & 15 & \\
\hline l. & $\mathrm{r}$ & 1. & $\mathrm{r}$ & 1. & r. & 1. & r. & 1. & r. & 1. & r. & 1. & r. \\
\hline 559 & 572 & 627 & 633 & 453 & 460,5 & 645,5 & 627 & 517 & 507 & 609,5 & 605,5 & 603 & 584 \\
\hline 354 & 366 & 415 & 424 & 283 & 281,5 & 399 & 399 & 322 & 325 & 388,5 & 388 & 382,5 & 382 \\
\hline 1,58 & 1,56 & 1,51 & 1,50 & 1,60 & 1,63 & 1,62 & 1,60 & 1,61 & 1,56 & 1,57 & 1,57 & 1,58 & $3 \quad 1,503$ \\
\hline 258 & 257 & 265 & 261 & 249 & 245 & 258 & 261 & 251 & 251 & 257 & 260 & 252 & 252 \\
\hline 31,5 & 31,9 & 32,8 & 33,4 & 28,7 & 33,3 & 32 & 31,4 & 30 & 29,8 & 31,3 & 31,1 & 31,8 & 31,5 \\
\hline 44,1 & 44,3 & 44 & 44,5 & 39,2 & 39 & ca. 44 & ca. 45 & 41,6 & 41,3 & 41,6 & 42,1 & 43,2 & 43,2 \\
\hline 184 & 182 & 195 & 191 & 176 & 175 & 186 & 185 & 180 & 181 & 184 & 185 & 182 & 178 \\
\hline 181 & 178 & 195 & 199 & 175 & 175 & 180 & 178 & 180 & 176 & 186 & 187 & 182 & 179 \\
\hline 20 & 18 & 14 & 26 & 47 & 35 & 12 & 32 & 12 & 28 & 18 & 22 & 23 & 25 \\
\hline 21 & 16 & 25 & 25 & 28 & 25 & 12 & 29 & 18 & 26 & 18 & 26 & 29 & 26 \\
\hline 19 & 19 & 15 & 28 & 23 & 22 & 14 & 23 & 18 & 25 & 21 & 20 & 30 & 21 \\
\hline 19 & 16 & 15 & 23 & 22 & 18 & 16 & 19 & 18 & 23 & 18 & 21 & 30 & 22 \\
\hline 19 & 18 & 27 & 25 & 21 & 20 & 19 & 20 & 20 & 25 & 18 & 20 & 25 & 21 \\
\hline 16 & 16 & 29 & 23 & 21 & 22 & 16 & 21 & 21. & 22 & 18 & 18 & 22 & 21 \\
\hline 19 & 14 & 22 & 25 & 21 & 21 & 16 & 20 & 21 & 22 & 18 & 20 & 15 & 21 \\
\hline 16 & 18 & 18 & 25 & 19 & 28 & 19 & 18 & 20 & 22 & 19 & 18 & 25 & 22 \\
\hline 19 & 16 & 21 & 23 & 20 & 34 & 19 & 19 & 20 & 23 & 19 & 19 & 33 & 20 \\
\hline 18 & 19 & 36 & 23 & 28 & 28 & 21 & 19 & 20 & 25 & 21 & 16 & 26 & 19 \\
\hline 18 & 18 & 25 & 25 & 29 & - & 22 & 21 & - & 26 & 18 & 19 & 35 & 27 \\
\hline 21 & 20 & 22 & 26 & 27 & - & 29 & 19 & - & 25 & 20 & 22 & 40 & 29 \\
\hline 23 & 18 & 33 & 27 & 26 & - & - & 21 & - & - & 20 & - & 43 & 34 \\
\hline- & - & - & - & - & - & - & 23 & - & - & - & - & - & - \\
\hline- & - & - & - & - & - & - & - & - & - & - & - & - & - \\
\hline- & - & - & - & - & - & - & - & - & - & - & - & - & - \\
\hline- & - & - & - & - & - & - & - & - & - & - & - & - & - \\
\hline- & - & - & - & - & - & - & - & - & - & - & - & - & - \\
\hline 1580 & 1540 & 1460 & 1570 & 1300 & 1320 & 1710 & 1690 & 1370 & 1310 & 1520 & 1560 & 1680 & 1510 \\
\hline- & - & - & - & - & - & - & - & - & - & - & - & - & - \\
\hline- & - & - & - & - & - & - & - & - & - & - & - & - & - \\
\hline- & - & - & - & - & - & - & - & - & - & - & - & - & - \\
\hline
\end{tabular}


Noch Tabelle I (Haupttabelle).

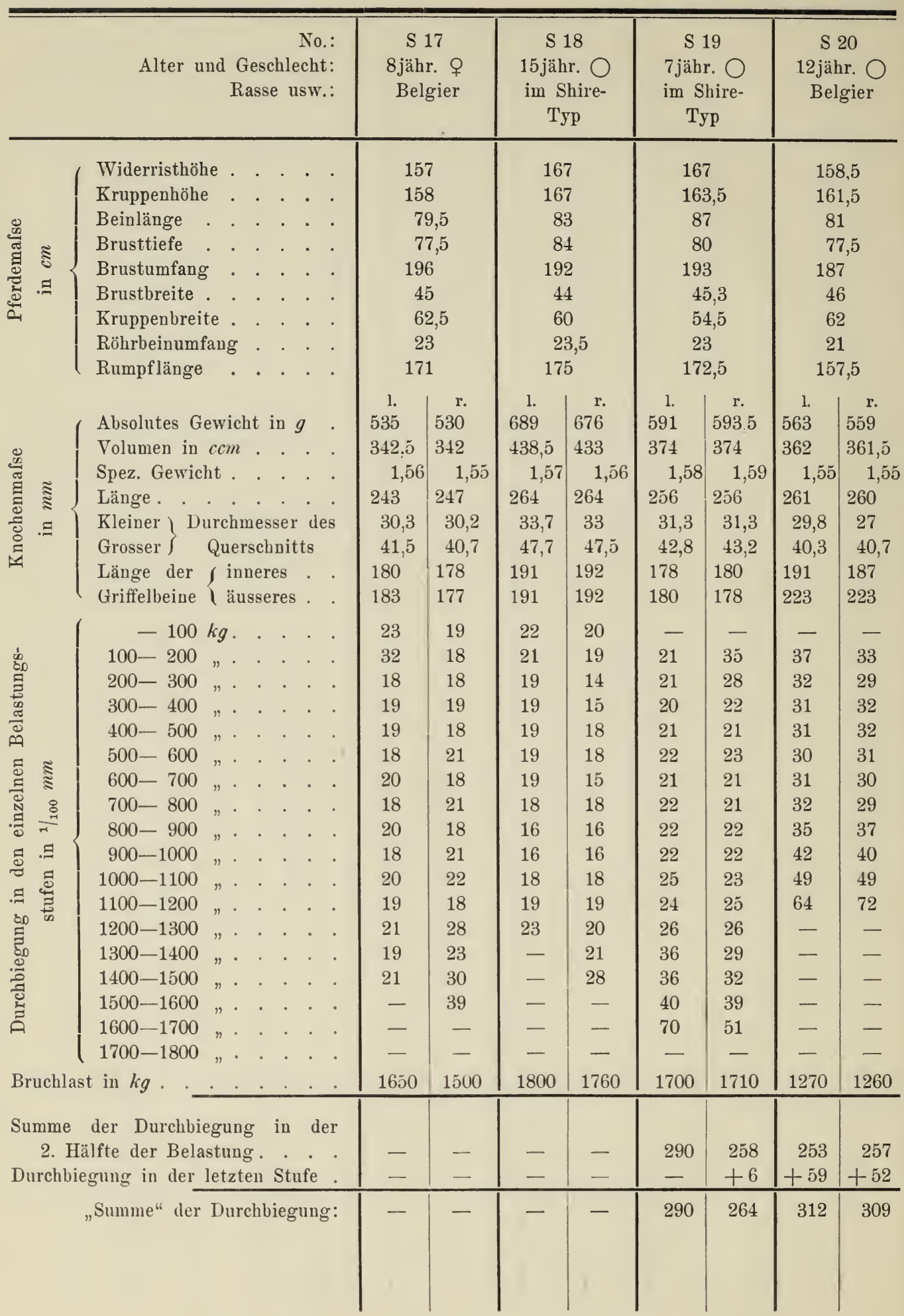


Noch Tabelle I (Haupttabelle).

\begin{tabular}{|c|c|c|c|c|c|c|c|c|c|c|c|c|c|}
\hline \multicolumn{2}{|c|}{$\begin{array}{c}\text { S } 21 \\
\text { 15jähr. ㅇ } \\
\text { Belgier }\end{array}$} & \multicolumn{2}{|c|}{$\begin{array}{c}\text { S } 22 \\
\text { 16 jähr. ㅇ } \\
\text { Belgier }\end{array}$} & \multicolumn{2}{|c|}{$\begin{array}{c}\text { S } 23 \\
\text { 16jähr. } \bigcirc \\
\text { Belgier }\end{array}$} & \multicolumn{2}{|c|}{$\begin{array}{c}\text { S } 24 \\
\text { 12jähr. } \bigcirc \\
\text { Belgier }\end{array}$} & \multicolumn{2}{|c|}{$\begin{array}{c}\text { S } 25 \\
\text { 15jähr. } \bigcirc \\
\text { Belgier }\end{array}$} & \multicolumn{2}{|c|}{$\begin{array}{c}\text { S } 26 \\
\text { 15̃jähr. } \bigcirc \\
\text { Belgier }\end{array}$} & \multicolumn{2}{|c|}{$\begin{array}{c}\text { S } 27 \\
\text { 9jähr. } \bigcirc \\
\text { Belgier }\end{array}$} \\
\hline \multicolumn{2}{|c|}{161} & \multicolumn{2}{|c|}{156,5} & \multicolumn{2}{|c|}{153} & \multicolumn{2}{|c|}{170} & \multicolumn{2}{|c|}{169,5} & \multicolumn{2}{|c|}{168} & \multicolumn{2}{|c|}{157} \\
\hline \multicolumn{2}{|c|}{161,5} & \multicolumn{2}{|c|}{158} & \multicolumn{2}{|c|}{152} & \multicolumn{2}{|c|}{167,5} & \multicolumn{2}{|c|}{169} & \multicolumn{2}{|c|}{164} & \multicolumn{2}{|c|}{160} \\
\hline \multirow{2}{*}{\multicolumn{2}{|c|}{$\begin{array}{l}85 \\
76\end{array}$}} & 76 & & 76 & & 84 & & 9 & & 8 & 4,5 & 8 & \\
\hline & & 80 & & 7 & & 86 & & 78 & 3,5 & 8 & 3,5 & 76 & \\
\hline $19:$ & & 182 & & 178 & & 193 & & 19 & & 196 & & 18 & \\
\hline 4 & 5,5 & 43 & & 4 & & 42 & & 4 & & 4 & & 4 & 3,5 \\
\hline $5 \varepsilon$ & 8,5 & 59 & & 6 & & 63 & 3,5 & 7 & & 60 & & 58 & 8,5 \\
\hline & 1,8 & 22 & & 2 & & & 3,5 & 2 & & & 2,5 & & 3,5 \\
\hline 160 & & 163 & & 16 & 7,5 & 171 & & 17 & & 174 & & 17 & \\
\hline 1. & r. & 1. & r. & 1. & r. & 1. & $\mathrm{r}$. & 1. & r. & 1. & r. & 1. & r. \\
\hline 551 & 553 & 559 & 561 & 640 & 639 & 569 & 573,5 & 724,5 & 726 & 625 & 617 & 470,5 & 501,5 \\
\hline 333 & 333 & 355 & 359 & 403,5 & 405,5 & 354,5 & 367 & 449 & 453 & 382 & 376,5 & 298 & 307,5 \\
\hline 1,65 & 1,66 & 1,57 & 1,56 & 1,58 & 1,57 & 1,60 & 1,56 & 1,61 & 1,60 & 1,63 & 1,64 & 1,58 & 1,63 \\
\hline 256 & 256 & 250 & 250 & 252 & 250 & 246 & 248 & 274 & 277 & 265 & 267 & 254 & 255 \\
\hline 29,7 & 28,7 & 30,8 & 31 & 31,5 & 32,1 & 31,3 & 31,6 & 31,8 & 31,5 & 31,4 & 31,8 & 28 & 28 \\
\hline 41,6 & 41,4 & 42,6 & 42,3 & 45,4 & 45,9 & ca. 46 & ca. 46 & 44,4 & 42 & 44,3 & 43,8 & 38 & 38,6 \\
\hline 175 & 180 & 178 & 182 & 188 & 189 & 178 & 179 & 193 & 198 & 187 & 191 & 181 & 183 \\
\hline 178 & 178 & 180 & 182 & 187 & 185 & 180 & 180 & 206 & 205 & 195 & 189 & 178 & 179 \\
\hline - & - & - & - & - & - & - & - & - & - & - & - & - & - \\
\hline 29 & 28 & 33 & 34 & 25 & 23 & 34 & 31 & 28 & 30 & 30 & 26 & 37 & 30 \\
\hline 26 & 24 & 31 & 31 & 20 & 20 & 29 & 27 & 25 & 28 & 27 & 25 & 40 & 30 \\
\hline 24 & 25 & 29 & 31 & 20 & 20 & 29 & 26 & 21 & 26 & 26 & 25 & 28 & 29 \\
\hline 24 & 26 & 29 & 28 & 20 & 20 & 30 & 25 & 22 & 26 & 24 & 24 & 32 & 28 \\
\hline 26 & 25 & 29 & 26 & 19 & 18 & 28 & 25 & 23 & 25 & 25 & 23 & 35 & 30 \\
\hline 24 & 26 & 32 & 25 & 20 & 19 & 30 & 25 & 21 & 25 & 24 & 22 & 36 & 31 \\
\hline 26 & 37 & 30 & 27 & 21 & 20 & 34 & 24 & 22 & 24 & 24 & 22 & 37 & 32 \\
\hline 27 & 28 & 32 & 27 & 20 & 20 & $\left.36^{1}\right)$ & 25 & 24 & 25 & 25 & 21 & 43 & 36 \\
\hline 30 & 31 & 32 & 26 & 21 & 21 & 23 & 25 & 23 & 25 & 26 & 22 & 53 & 42 \\
\hline 35 & 35 & 34 & 31 & 23 & 22 & 24 & 27 & 24 & 28 & 27 & 23 & 78 & 53 \\
\hline 41 & 41 & 37 & 34 & 24 & 24 & 29 & 31 & 27 & 29 & 33 & 24 & - & 69 \\
\hline 51 & 51 & 44 & 43 & 27 & 26 & 31 & 35 & 28 & 35 & 36 & 26 & - & - \\
\hline- & - & $120 ?$ & 57 & 31 & 30 & 40 & 44 & 34 & 36 & 37 & 27 & - & - \\
\hline- & - & 51 & 72 & 37 & 37 & 52 & 66 & 38 & 45 & 50 & 32 & - & - \\
\hline- & - & - & - & 43 & 45 & - & - & 58 & 57 & - & 49 & - & - \\
\hline- & - & - & - & 53 & 73 & - & - & - & - & - & 44 & - & - \\
\hline- & - & - & - & - & - & - & - & - & - & - & 42 & - & - \\
\hline 1330 & 1320 & 1560 & 1520 & 1710 & 1700 & 1560 & 1550 & 1640 & 1605 & 1540 & 1860 & 1140 & 1270 \\
\hline $\begin{array}{r}222 \\
+20 \\
\end{array}$ & $\begin{array}{r}236 \\
+14 \\
\end{array}$ & $\begin{array}{r}365 \\
+58 \\
\end{array}$ & $\begin{array}{r}304 \\
+16 \\
\end{array}$ & $\begin{array}{r}269 \\
+6 \\
\end{array}$ & $\begin{array}{l}288 \\
- \\
\end{array}$ & $\begin{array}{r}252 \\
+40 \\
\end{array}$ & $\begin{array}{r}265 \\
+35 \\
\end{array}$ & $\begin{array}{r}256 \\
+26 \\
\end{array}$ & $\begin{array}{r}280 \\
+4 \\
\end{array}$ & $\begin{array}{r}246 \\
+26 \\
\end{array}$ & $\begin{array}{r}289 \\
+39 \\
\end{array}$ & $\begin{array}{r}265 \\
+39 \\
\end{array}$ & $\begin{array}{r}263 \\
+61 \\
\end{array}$ \\
\hline 242 & 250 & 423 & 320 & 275 & 288 & 292 & 300 & 282 & 284 & 272 & 328 & 304 & 324 \\
\hline
\end{tabular}

1) Krachen des Knochens. 
Noch Tabelle I (Haupttabelle).

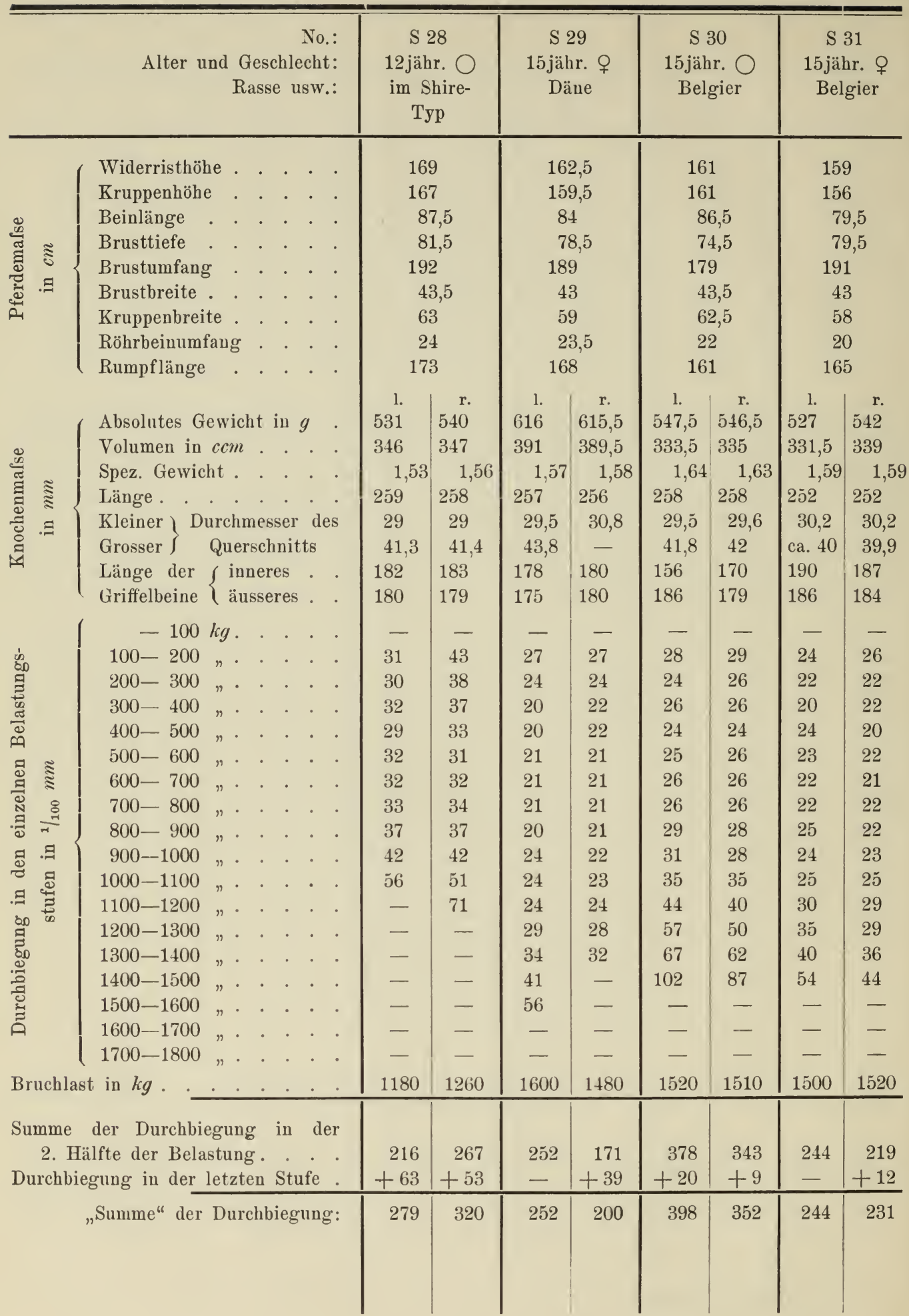


Noch Tabelle I (Haupttabelle).

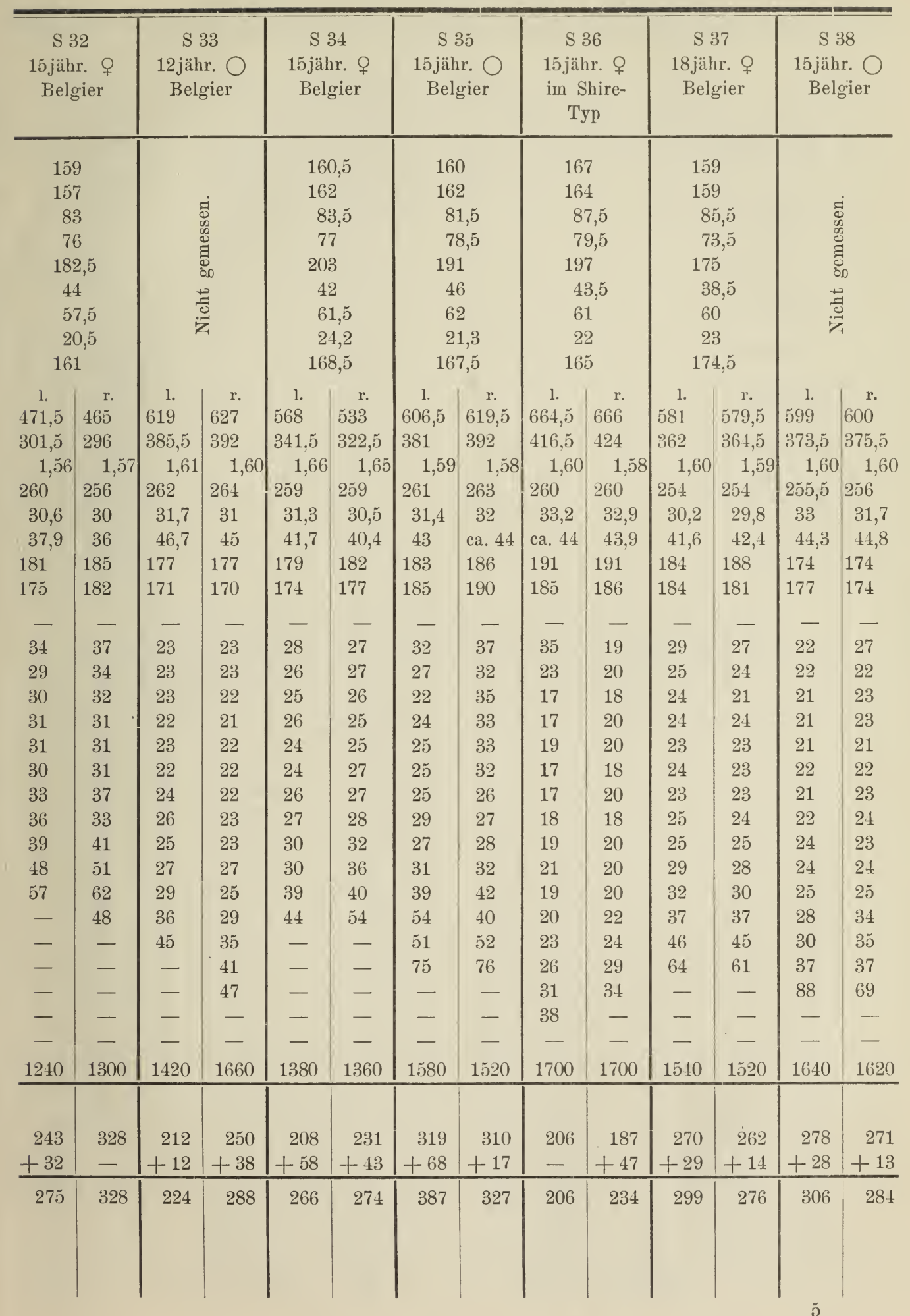


Tabelle II. Zusammenstellung der Pferdemalse.

Durchschnitt von 59 Laufpferden und 38 Schrittpferden.

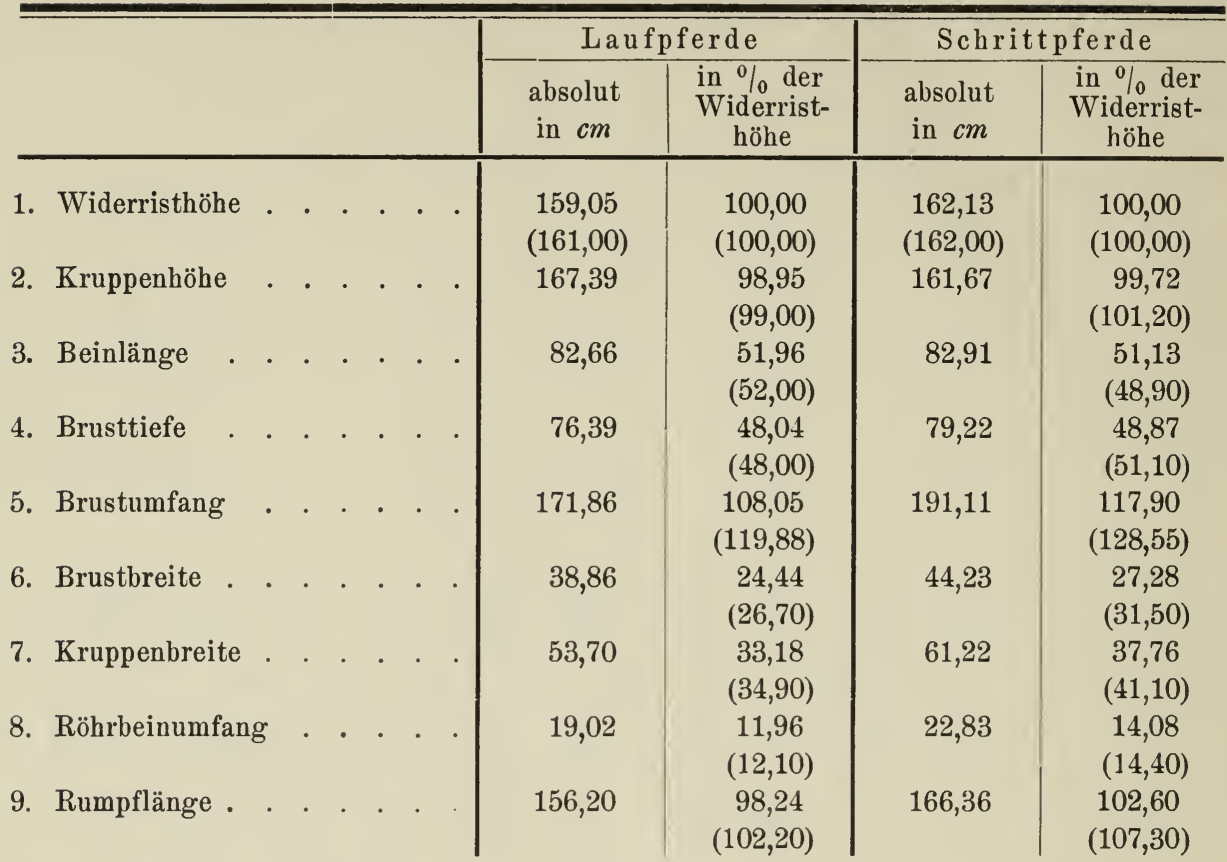

Tabelle III. Verhältnis von Röhrbeinlänge zu Widerristhöhe.

Die Röhrbeinlänge beträgt von der Widerristhöhe nachfolgende Prozente.

\begin{tabular}{|c|c|c|c|c|c|c|c|c|c|}
\hline \multicolumn{6}{|c|}{ Bei Laufpferden: } & \multicolumn{4}{|c|}{ Bei Schrittpferden: } \\
\hline No. & $\%$ & No. & $\%$ & No. & $\%$ & No. & $\%$ & No. & $\%$ \\
\hline 1 & - & 22 & 17,0 & 43 & 15,3 & 1 & 16,2 & 22 & 16,0 \\
\hline 2 & 15,9 & 23 & 16,2 & 44 & 16,0 & 2 & 16,5 & 23 & 16,4 \\
\hline 3 & 16,0 & 24 & 16,4 & 45 & 16,2 & 3 & 16,2 & 24 & 14,5 \\
\hline 4 & 17,9 & 25 & 15,7 & 46 & 16,6 & 4 & 15,8 & 25 & 16,3 \\
\hline 5 & 15,6 & 26 & 16,1 & 47 & 16,7 & 5 & 16,0 & 26 & 15,9 \\
\hline 6 & 16,3 & 27 & 16,2 & 48 & 16,5 & 6 & 15,5 & 27 & 16,2 \\
\hline 7 & 16,5 & 28 & 16,2 & 49 & 16,1 & 7 & 15,8 & 28 & 15,3 \\
\hline 8 & 15,7 & 29 & 16,1 & 50 & 16,2 & 8 & 15,3 & 29 & 15,8 \\
\hline 9 & 16,8 & 30 & 16,2 & 51 & 16,2 & 9 & 16,5 & 30 & 16,0 \\
\hline 10 & 16,2 & 31 & 16,3 & 52 & 16,8 & 10 & 16,0 & 31 & 15,8 \\
\hline 11 & 16,4 & 32 & 16,6 & 53 & 16,7 & 11 & 16,0 & 32 & 16,2 \\
\hline 12 & - & 33 & 16,7 & 54 & 16,5 & 12 & 16,0 & 33 & - \\
\hline 13 & 15,6 & 34 & 16,2 & 55 & 16,0 & 13 & 15,4 & 34 & 16,1 \\
\hline 14 & 16,2 & 35 & 16,1 & 56 & 16,7 & 14 & 15,6 & 35 & 16,4 \\
\hline 15 & 15,9 & 36 & 16,7 & 57 & 16,6 & 15 & 15,7 & 36 & 15,6 \\
\hline 16 & 17,0 & 37 & 16,6 & 58 & 15,7 & 16 & 16,0 & 37 & 16,0 \\
\hline 17 & 16,3 & 38 & 16,2 & 59 & 17,0 & 17 & 15,6 & 38 & - \\
\hline 18 & 17,4 & 39 & 16,2 & $(60$ & $17,5)$ & 18 & 15,8 & & \\
\hline 19 & 15,9 & 40 & 15,6 & (61 & $18,0)$ & 19 & 15,3 & & \\
\hline 20 & 16,4 & 41 & 16,6 & & & 20 & 16,4 & & \\
\hline 21 & 16,3 & 42 & 16,2 & & & 21 & 15,9 & & \\
\hline
\end{tabular}


Tabelle IV L. Maise des Querschnitts.

Laufpferde.

\begin{tabular}{|c|c|c|c|c|c|c|c|c|c|c|c|c|}
\hline \multirow[b]{2}{*}{ No. } & \multicolumn{6}{|c|}{ Lin $\mathrm{ks}$ : } & \multicolumn{6}{|c|}{ Rechts: } \\
\hline & 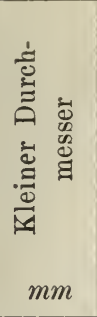 & 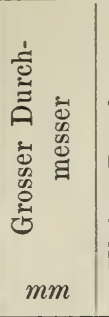 & 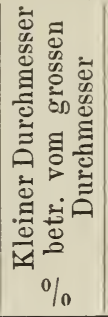 & 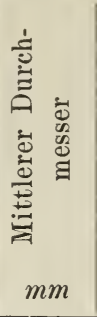 & 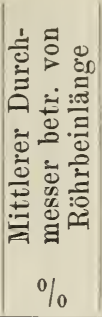 & 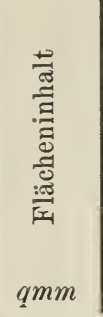 & 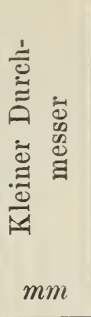 & 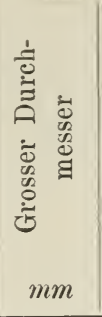 & 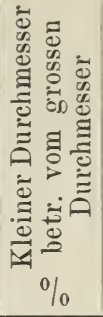 & 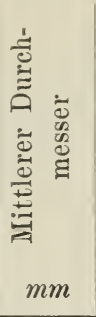 & 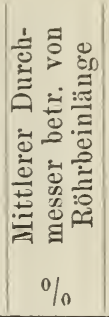 & 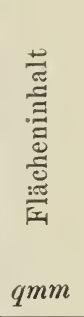 \\
\hline 1 & 28,0 & 36,0 & 78 & 32,0 & 12,5 & 792 & 28,0 & 35,9 & 78 & 32,0 & 12,4 & 790 \\
\hline 2 & 27,3 & 35,0 & 78 & 31,1 & 13,0 & 750 & 27,0 & 34,5 & 78 & 30,7 & 12,8 & 731 \\
\hline 3 & 28,8 & 35,4 & 81 & 32,1 & 12,6 & 801 & 29,3 & 35,4 & 83 & 32,3 & 12,6 & 815 \\
\hline 4 & 28,0 & 35,1 & 80 & 31,5 & 12,5 & 772 & 28,7 & 35,3 & 81 & 32,0 & 12,6 & 796 \\
\hline 5 & 27,7 & 36,2 & 77 & 31,9 & 12,8 & 788 & 26,7 & 38,4 & 70 & 32,5 & 13,1 & $80 ら$ \\
\hline 6 & 26,9 & 35,9 & 75 & 31,4 & $12, \check{\partial}$ & 759 & 27,6 & 36,6 & 75 & 52,1 & 12,8 & 794 \\
\hline 7 & 30,4 & 38,7 & 79 & 34,5 & 13,4 & 924 & 30,2 & 39,4 & 77 & 34,8 & 13,4 & 935 \\
\hline 8 & 29,0 & 38,0 & 76 & 33,5 & 13,5 & 866 & 29,0 & 37,8 & 77 & 33,4 & 13,5 & 861 \\
\hline 9 & 29,2 & 37,7 & 77 & 33,4 & 12,9 & 865 & 28,9 & $3 \check{5}, 4$ & 82 & 32,1 & 12,4 & 804 \\
\hline 10 & 27,5 & 36,8 & 75 & 32,1 & 13,1 & 795 & 26,7 & 35,1 & 76 & 30,9 & 12,6 & 736 \\
\hline 11 & 28,1 & 35,2 & 80 & 31,6 & 12,7 & 777 & 28,5 & 37,7 & 76 & 33,1 & 13,3 & 844 \\
\hline 12 & 33,6 & 40,2 & 83 & 36,9 & 13,2 & 1062 & 33,5 & 40,8 & 82 & 37,1 & 13,3 & 1074 \\
\hline 13 & 29,4 & 37,2 & 79 & 33,3 & 13,1 & 860 & 29,5 & 37,2 & 79 & 33,3 & 13,2 & 862 \\
\hline 14 & 26,8 & 34,8 & 77 & 30,8 & 12,7 & 733 & 25,6 & 34,8 & 74 & 30,2 & 12,1 & 700 \\
\hline 15 & 26,8 & 37,8 & 71 & 32,3 & 13,1 & 796 & 26,2 & 37,0 & 71 & 31,6 & 12,8 & 761 \\
\hline 16 & 30,0 & 38,1 & 79 & 34,0 & 12,9 & 858 & 29,2 & 38,6 & 76 & 33,9 & 12,8 & 885 \\
\hline 17 & 29,4 & 39,2 & 75 & 34,3 & 13,2 & 905 & 30,5 & 39,0 & 78 & 34,7 & 13,3 & 934 \\
\hline 18 & 30,8 & 39,0 & 79 & 34,9 & 13,1 & 944 & 32,3 & 40,0 & 81 & 36,1 & 13,6 & 1015 \\
\hline 19 & 25,7 & 35,2 & 73 & 30,4 & 12,3 & 711 & 26,4 & 35,4 & 75 & 30,9 & 12,5 & 738 \\
\hline 20 & 26,0 & 36,5 & 71 & 31,2 & 12,6 & 746 & 26,8 & 36,4 & 74 & 31,6 & 12,8 & 767 \\
\hline 21 & 25,0 & 31,0 & 81 & 28,0 & 11,5 & 609 & 26,2 & 31,6 & 83 & 28,9 & 11,8 & 651 \\
\hline 22 & 26,7 & 35,0 & 76 & 30,8 & 12,1 & 734 & 27,3 & $3 \tilde{s}, \tilde{5}$ & 77 & 31,4 & 12,3 & 761 \\
\hline 23 & 31,4 & 37,0 & 85 & 34,2 & 13,0 & 913 & $31, \overline{5}$ & 37,3 & 84 & 34,4 & 13,0 & 923 \\
\hline 24 & 25,0 & 33,6 & 74 & 29,3 & 11,9 & 660 & 26,0 & 33,3 & 78 & 29,6 & 12,0 & 680 \\
\hline 25 & 28,5 & 37,0 & 77 & 32,7 & 13,3 & 828 & 29,0 & 37,0 & 78 & 33,0 & 13,5 & 843 \\
\hline 26 & 26,3 & 35,0 & 75 & 30,6 & 12,6 & 723 & 26,6 & 35,0 & 76 & 30,8 & 12,7 & 731 \\
\hline 27 & 29,0 & 39,4 & 74 & 34,2 & 13,5 & 898 & 30,0 & 37,6 & 80 & 33,8 & 13,3 & 886 \\
\hline 28 & 27,0 & 38,3 & 70 & 32,6 & 12,7 & 812 & 27,3 & 38,0 & 72 & 32,6 & 12,5 & 815 \\
\hline 29 & 25,0 & 35,7 & 70 & 30,3 & 12,1 & 701 & 25,0 & 36,0 & 69 & 30,5 & 12,2 & 707 \\
\hline 30 & 27,8 & 35,2 & 79 & 31,5 & 12,3 & 769 & 28,3 & 36,0 & 79 & 32,1 & 12,6 & 801 \\
\hline 31 & 30,0 & 37,4 & 80 & 33,7 & 12,5 & 882 & 30,0 & 36,5 & 82 & 33,2 & 12,3 & 860 \\
\hline 32 & 26,5 & 35,4 & 75 & 30,9 & 12,1 & 737 & 27,3 & 36,0 & 76 & 31,6 & 12,4 & 772 \\
\hline 33 & 27,9 & 35,8 & 78 & 31,8 & 12,6 & 785 & 27,7 & 35,4 & 78 & 31,5 & 12,6 & 771 \\
\hline werrtagen. & 925,5 & 1203,8 & 2537 & 1063,8 & 419,9 & 27832 & 932,8 & 1205,9 & 2555 & 1068,7 & 421,2 & 28071 \\
\hline
\end{tabular}


Noch Tabelle IV L.

\begin{tabular}{|c|c|c|c|c|c|c|c|c|c|c|c|c|}
\hline \multirow[b]{2}{*}{ No. } & \multicolumn{6}{|c|}{ L in k s: } & \multicolumn{6}{|c|}{$\mathrm{Rechts:}$} \\
\hline & 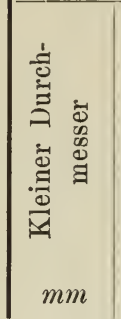 & 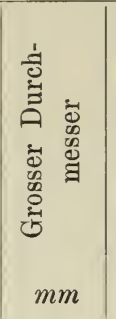 & 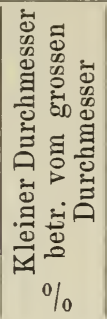 & 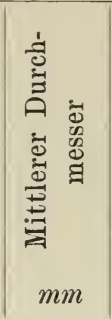 & 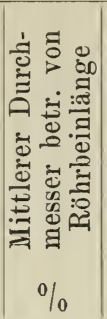 & 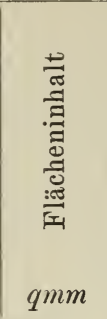 & 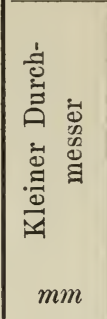 & 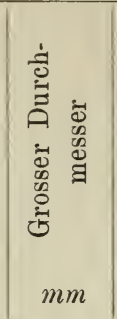 & 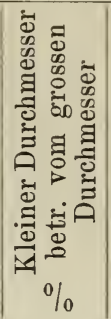 & 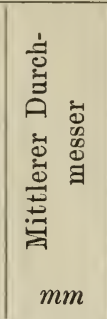 & 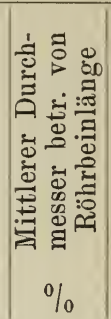 & 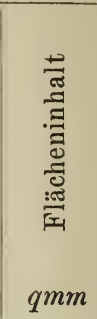 \\
\hline Übertrag: & 925,5 & 1203,8 & 2537 & 1063,8 & 419,9 & 27832 & 932,8 & 1205,9 & 2555 & 1068,7 & 421,2 & 28071 \\
\hline 34 & 26,7 & 35,8 & 75 & 31,2 & 13,0 & 751 & 26,7 & 37,0 & 72 & 31,8 & 13,3 & 776 \\
\hline 35 & 27,6 & 35,5 & 78 & 31,5 & 13,3 & 770 & 28,0 & 35,5 & 79 & 31,7 & 13,3 & 781 \\
\hline 36 & 24,4 & 33,0 & 74 & 28,7 & 11,5 & 632 & 26,3 & 32,8 & 80 & 29,5 & 11,8 & 678 \\
\hline 37 & 27,3 & 36,0 & 76 & 31,6 & 12,5 & 772 & 27,0 & 35,8 & 75 & 31,4 & 12,4 & 759 \\
\hline 38 & 28,6 & 38,0 & 75 & 33,3 & 13,1 & 854 & 28,7 & 37,5 & 77 & 33,1 & 12,9 & 838 \\
\hline 39 & 28,7 & 36,8 & 78 & 32,7 & 13,1 & 830 & 28,2 & 37,2 & 76 & 32,7 & 13,1 & 824 \\
\hline 40 & 27,0 & 36,4 & 74 & 31,7 & 13,0 & 772 & 27,0 & 36,0 & 75 & 31,5 & 13,0 & 764 \\
\hline 41 & 30,0 & 37,0 & 81 & 33,5 & 12,3 & 872 & 29,8 & 37,2 & 80 & 33,5 & 12,2 & 871 \\
\hline 42 & 26,3 & 33,7 & 78 & 30,0 & 12,2 & 696 & 25,8 & 32,8 & 79 & 29,3 & 11,7 & 665 \\
\hline 43 & 26,7 & 35,4 & 75 & 31,0 & 12,8 & 742 & 26,5 & 35,5 & 75 & 31,0 & 12,4 & 739 \\
\hline 44 & 28,0 & 36,4 & 77 & 32,2 & 13,0 & 801 & 28,7 & 37,5 & 77 & 33,1 & 13,4 & 845 \\
\hline 45 & 27,4 & 37,3 & 73 & 32,3 & 12,5 & 803 & 26,6 & 37,3 & 71 & 31,9 & 12,4 & 779 \\
\hline 46 & 28,7 & 37,2 & 77 & 32,9 & 12,3 & 839 & 28,7 & 36,8 & 78 & 32,7 & 12,3 & 830 \\
\hline 47 & 29,5 & 36,3 & 81 & 32,9 & 12,4 & 841 & 31,3 & 37,7 & 83 & 34,5 & 13,0 & 927 \\
\hline 48 & 28,6 & 37,5 & 76 & 33,0 & 12,5 & 842 & 28,4 & 38,2 & 74 & 33,3 & 12,5 & 852 \\
\hline 49 & 28,6 & 36,8 & 78 & 32,7 & 12,7 & 827 & 29,0 & 37,8 & 77 & 33,4 & 13,0 & 861 \\
\hline 50 & 28,5 & 35,4 & 81 & 31,9 & 12,6 & 793 & 28,2 & 35,8 & 79 & 32,0 & 12,8 & 793 \\
\hline 51 & 27,4 & 37,4 & 73 & 32,4 & 12,6 & 805 & 27,4 & 37,5 & 73 & 32,4 & 12,6 & 807 \\
\hline 52 & 27,0 & 34,6 & 78 & 30,8 & 12,3 & 734 & 26,7 & 34,4 & 78 & 30,5 & 12,2 & 721 \\
\hline 53 & 26,8 & 34,2 & 78 & 30,5 & 11,8 & 720 & 27,4 & 34,2 & 80 & 30,8 & 12,0 & 736 \\
\hline 54 & 28,6 & 38,7 & 74 & 33,6 & 13,2 & 870 & 30,0 & 38,3 & 78 & 34,1 & 13,4 & 903 \\
\hline 55 & 27,8 & 38,0 & 73 & 32,9 & 13,5 & 829 & 27,2 & 38,4 & 71 & 32,8 & 13,4 & 820 \\
\hline 56 & 28,7 & 39,3 & 73 & 34,0 & 12,9 & 886 & 28,7 & 39,6 & 72 & 34,1 & 12,9 & 893 \\
\hline 57 & 28,0 & 37,0 & 76 & 32,5 & 12,0 & 814 & 27,4 & 37,6 & 73 & 32,5 & 12,0 & 810 \\
\hline 58 & 29,6 & 40,0 & 74 & 34,8 & 13,3 & 930 & 30,0 & 40,8 & 74 & 35,4 & 13,5 & 962 \\
\hline 59 & 28,0 & 37,7 & 74 & 32,8 & 12,2 & 830 & 28,0 & 37,3 & 75 & 32,6 & 12,2 & 820 \\
\hline$(60)$ & 23,0 & 29,6 & 77 & 26,3 & - & 535 & 22,5 & 29,5 & 76 & 26,0 & - & 522 \\
\hline$(61)$ & 26,1 & 34,2 & 76 & 30,1 & - & 702 & 26,3 & 34,4 & 76 & 30,3 & - & 711 \\
\hline Summe: & 1650,0 & 2155,2 & 4517 & |1901,2 & 748,5 & 48487 & 1660,5 & 2162,4 & 4538 & 1910,3 & 750,9 & 49127 \\
\hline je: & $\mid 27,97$ & $|36,53|$ & 76,57 & 32,22 & $|12,68|$ & 821,81 & 28,14 & 36,65 & 76,91 & 32,38 & 12,73 & 832,66 \\
\hline & Durchsch & hnitt von & n links & und recl & hts zusa & ammen: & 28,06 & 36,59 & 76,74 & 32,30 & 12,71 & 827,24 \\
\hline
\end{tabular}


Tabelle IV S. Maise des Querschnittes.

\section{Schrittpferde.}

\begin{tabular}{|c|c|c|c|c|c|c|c|c|c|c|c|c|}
\hline \multirow[b]{2}{*}{ No. } & \multicolumn{6}{|c|}{ L in ks: } & \multicolumn{6}{|c|}{ Rechts: } \\
\hline & 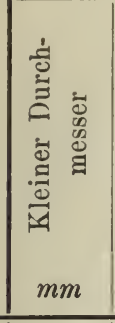 & 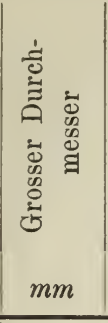 & 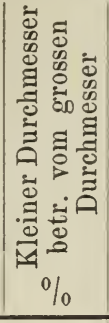 & 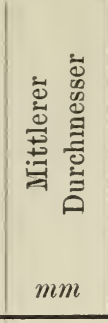 & 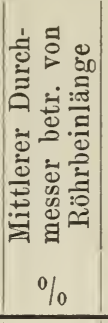 & 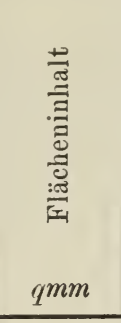 & 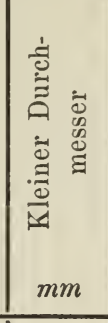 & 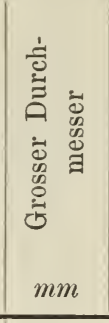 & 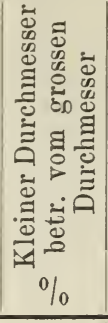 & 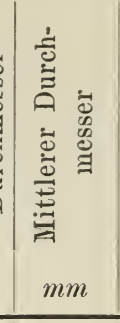 & 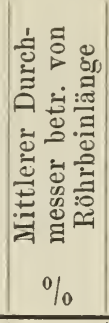 & 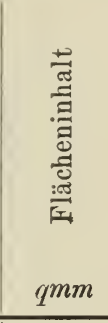 \\
\hline 1 & 27,9 & 36,5 & 76 & 32,2 & 12,5 & 800 & 27,2 & 36,6 & 74 & 31,9 & 12,3 & 783 \\
\hline 2 & 31,9 & 43,8 & 73 & 37,8 & 15,1 & 1098 & 31,5 & 42,3 & 74 & 36,9 & 14,6 & 1047 \\
\hline 3 & 32,1 & 44,8 & 72 & 38,4 & 14,3 & 1130 & 31,9 & 44,1 & 72 & 38,0 & 14,2 & 1105 \\
\hline 4 & 33,7 & 49,6 & 68 & 41,6 & 15,5 & 1314 & 34,7 & 47,1 & 74 & 40,9 & 15,3 & 1284 \\
\hline 5 & 30,8 & 42,6 & 72 & 36,7 & 14,2 & 1031 & 31,7 & 42,3 & 75 & 37,0 & 14,2 & 1060 \\
\hline 6 & 31,3 & 43,7 & 72 & 37,5 & 14,8 & 1075 & 30,8 & 44,7 & 69 & 37,7 & 14,7 & 1082 \\
\hline 7 & 31,2 & 43,4 & 72 & 37,3 & 14,6 & 1064 & 30,8 & 43,2 & 71 & 37,0 & 14,5 & 1046 \\
\hline 8 & 30,8 & 45,7 & 67 & 38,2 & 15,1 & 1106 & 30,4 & 45,5 & 67 & 37,9 & 15,0 & 1087 \\
\hline 9 & 32,8 & 43,8 & 75 & 38,3 & 14,5 & 1129 & 34,4 & 44,0 & 78 & 39,2 & 14,6 & 1190 \\
\hline 10 & 31,5 & 44,1 & 71 & 37,8 & 14,6 & 1091 & 31,9 & 44,3 & 72 & 38,1 & 14,8 & 1110 \\
\hline 11 & 32,8 & 44,0 & 75 & 38,4 & 14,5 & 1134 & 33,4 & $44, \check{5}$ & 75 & 38,9 & 14,9 & 1168 \\
\hline 12 & 28,7 & 39,2 & 73 & 33,9 & 13,6 & 884 & 28,3 & 39,0 & 85 & 36,1 & 14,7 & 1021 \\
\hline 13 & 32,0 & 44,0 & 73 & 38,0 & 14,7 & 1106 & 31,4 & 45,0 & 70 & 38,2 & 14,6 & 1110 \\
\hline 14 & 30,0 & 41,6 & 72 & 35,8 & 14,3 & 981 & 29,8 & 41,3 & 72 & 35,5 & 14,1 & 967 \\
\hline 15 & 31,3 & 41,6 & 75 & 36,4 & 14,2 & 1023 & 31,1 & 42,1 & 74 & 36,6 & 14,1 & 1028 \\
\hline 16 & 31,8 & 43,2 & 74 & 37,5 & 14,9 & 1080 & 31,5 & 43,2 & 73 & 37,3 & 14,8 & 1069 \\
\hline 17 & 30,3 & 41,5 & 73 & 35,9 & 14,8 & 988 & 30,2 & 40,7 & 74 & 35,4 & 15,0 & 966 \\
\hline 18 & 33,7 & 47,7 & 71 & 40,7 & 15,4 & 1263 & 33,0 & 47,5 & 69 & 40,2 & 15,2 & 1231 \\
\hline 19 & 31,3 & 42,8 & 73 & 37,0 & 14,5 & 10552 & 31,3 & 43,2 & 72 & 37,2 & 14,5 & 1062 \\
\hline 20 & 29,8 & 40,3 & 74 & 35,0 & 13,4 & 944 & 27,0 & 40,7 & 66 & 33,8 & 13,0 & 863 \\
\hline 21 & 29,7 & 41,6 & 71 & 35,6 & 14,0 & 971 & 28,7 & 41,4 & 69 & 35,0 & 13,7 & 933 \\
\hline 22 & 30,8 & 42,6 & 72 & 36,7 & 14,6 & 1031 & 31,0 & 42,3 & 73 & 36,6 & 14,6 & 1030 \\
\hline 23 & 31,5 & 45,4 & 69 & 38,4 & 15,3 & 1124 & 32,1 & 45,9 & 70 & 39,0 & 15,6 & 11557 \\
\hline 24 & 31,3 & 46,0 & 68 & 38,6 & 15,9 & 1131 & 31,6 & 46,0 & 69 & 38,8 & 15,6 & 1142 \\
\hline 25 & 31,8 & 44,4 & 72 & 38,1 & 13,9 & 1109 & 31,5 & 42,0 & 75 & 36,7 & 13,3 & 1039 \\
\hline 26 & 31,4 & 44,3 & 71 & 37,8 & 14,3 & 1093 & 31,8 & 43,8 & 73 & 37,8 & 14,2 & 1094 \\
\hline 27 & 28,0 & 38,0 & 74 & 33,0 & 13,0 & 836 & 28,0 & 38,6 & 73 & 33,3 & 13,0 & 849 \\
\hline 28 & 29,0 & 41,3 & 70 & 35,1 & 13,6 & 941 & 29,0 & 41,4 & 70 & 35,2 & 13,6 & 944 \\
\hline 29 & 29,5 & 43,8 & 67 & 36,6 & 14,2 & 1015 & 30,8 & - & - & - & - & - \\
\hline 30 & 29,5 & 41,8 & 71 & 35,6 & 13,8 & 969 & 29,6 & 42,0 & 70 & 35,8 & 13,9 & 977 \\
\hline 31 & 30,2 & 40,0 & 76 & 35,1 & 13,9 & 949 & 30,2 & 39,9 & 76 & 35,0 & 13,9 & 947 \\
\hline 32 & 30,6 & 37,9 & 81 & 34,2 & 13,2 & 911 & 30,0 & 36,0 & 83 & 33,0 & 12,9 & 849 \\
\hline 33 & 31,7 & 46,7 & 68 & 39,2 & 15,0 & 1163 & 31,0 & 45,0 & 69 & 38,0 & 14,5 & 1096 \\
\hline 34 & 31,3 & 41,7 & 75 & 36,5 & 14,1 & 1025 & 30,5 & 40,6 & 75 & 35,4 & 13,7 & 968 \\
\hline 35 & 31,4 & 43,0 & 73 & 37,2 & 14,2 & 1061 & 32,0 & 44,0 & 73 & 38,0 & 14,9 & 1106 \\
\hline 36 & 33,2 & 44,0 & 75 & 38,6 & 14,9 & 1148 & 32,9 & 43,9 & 75 & 38,4 & 14,8 & $113 \check{~}$ \\
\hline 37 & 30,2 & 41,6 & 73 & 35,9 & 14,1 & 987 & 29,8 & 42,4 & 70 & 36,1 & 14,2 & 993 \\
\hline 38 & 33,0 & 44,3 & 74 & 38,6 & 15,1 & 1149 & 31,7 & 44,8 & 71 & 38,2 & 14,9 & 1116 \\
\hline Summe: & 1179,8 & 1632,3 & 2751 & 1405,2 & 546,6 & 39906 & 1174,5 & 1581,3 & 2690 & 1363,1 & 530,4 & 38654 \\
\hline 0. & 31,05 & 42,96 & $|72,39|$ & | 36,97 & $\mid 14,38$ & 1050,16 & 30,91 & 42,74 & 72,70 & 36,85 & 14,34 & 1044,70 \\
\hline & Durchsc & chnitt $\mathbf{v}$ & on links & $\mathrm{s}$ und $\mathrm{re}$ & chts zus & sammen: & 30,98 & $42,8 \check{a}$ & 72,55 & 36,91 & 14,36 & 1047,43 \\
\hline
\end{tabular}


Tabelle V. Verhältnis der Stärke des

Laufpferde.

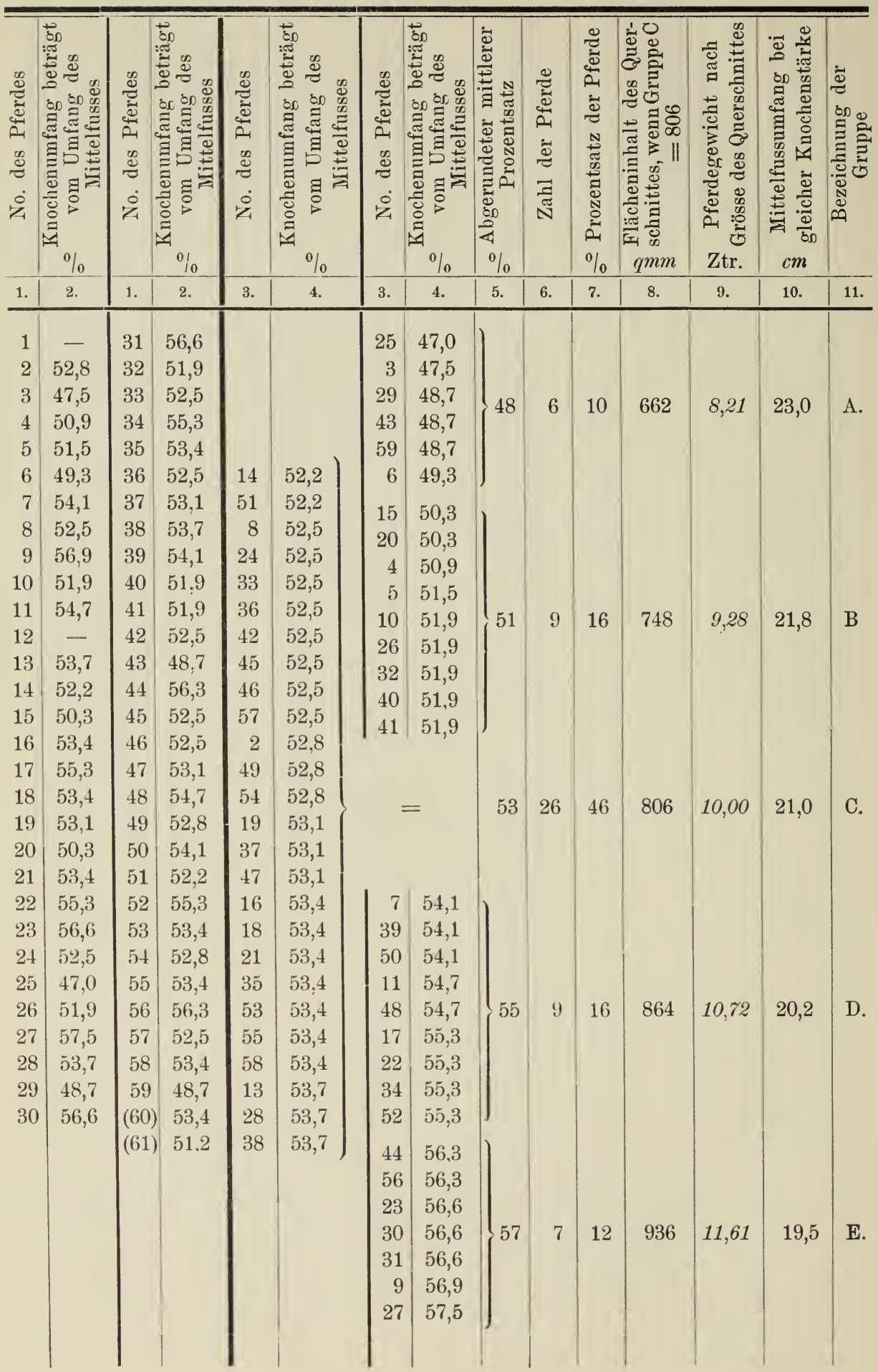


Mittelfusses gegenüber der des Metacarpus.

Schrittpferde.

\begin{tabular}{|c|c|c|c|c|c|c|c|c|c|c|}
\hline 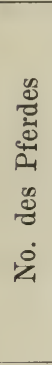 & 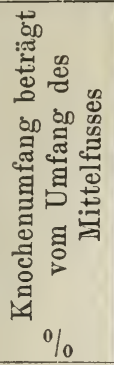 & 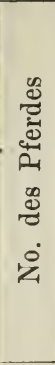 & 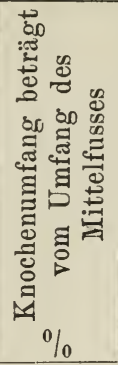 & 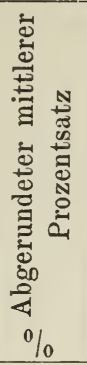 & 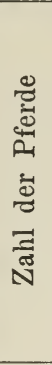 & 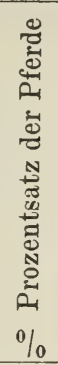 & 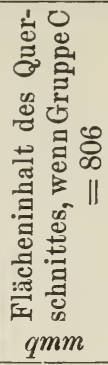 & 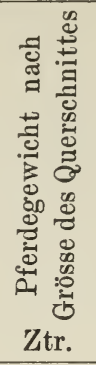 & 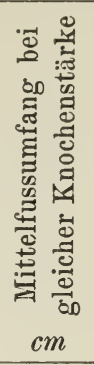 & 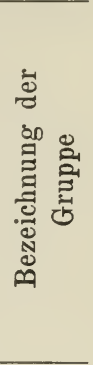 \\
\hline 1. & 2. & 3. & 4. & 5. & 6. & 7. & 8. & 9. & 10. & 11. \\
\hline 1 & 46,8 & 13 & 43,4 & & & & & & & \\
\hline 2 & 56,6 & 27 & 44,0 & & & & & & & \\
\hline 3 & 49,3 & 28 & 45,9 & 45 & 5 & 14 & 808 & 10,80 & 26,1 & A. \\
\hline 4 & 49,3 & 1 & 46,8 & & & & & & & \\
\hline 5 & 53,7 & 34 & 47,5 & & & & & & & \\
\hline 6 & 52,8 & & & & & & & & & \\
\hline 7 & 53,4 & 12 & 48,4 & & & & & & & \\
\hline 8 & 50,6 & 15 & $\begin{array}{l}48,7 \\
490\end{array}$ & & & & & & & \\
\hline 9 & 49,7 & $\begin{array}{l}17 \\
29\end{array}$ & $\begin{array}{l}49,0 \\
49,0\end{array}$ & & & & & & & \\
\hline 10 & 52,8 & $\begin{array}{l}29 \\
37\end{array}$ & $\begin{array}{l}49,0 \\
49,0\end{array}$ & 49 & 8 & 22 & 966 & 12,92 & 23,9 & B. \\
\hline 11 & 51,9 & 3 & $\begin{array}{l}4,0 \\
49,3\end{array}$ & & & & & & & \\
\hline 12 & $\begin{array}{l}48,4 \\
43,4\end{array}$ & 4 & 49,3 & & & & & & & \\
\hline 13 & $\begin{array}{l}43,4 \\
51,2\end{array}$ & 9 & 49,7 & & & & & & & \\
\hline 14 & $\begin{array}{l}51,2 \\
48,7\end{array}$ & 19 & & & & & & & & \\
\hline 15 & $\begin{array}{l}48,7 \\
52,5\end{array}$ & $\begin{array}{l}19 \\
23\end{array}$ & $\begin{array}{l}50,3 \\
50,3\end{array}$ & & & & & & & \\
\hline 16 & $\begin{array}{l}52,5 \\
49,0\end{array}$ & 8 & $\begin{array}{l}50,3 \\
50,6\end{array}$ & & & & & & & \\
\hline 17 & $\begin{array}{l}49,0 \\
54,4\end{array}$ & 30 & $\begin{array}{l}50,6 \\
50,9\end{array}$ & & & & & & & \\
\hline 18 & $\begin{array}{l}54,4 \\
50,3\end{array}$ & 14 & $\begin{array}{l}50,9 \\
51,2\end{array}$ & 51 & 8 & 22 & 1047 & 14,00 & 23,0 & C. \\
\hline 19 & $\begin{array}{l}50,3 \\
52,5\end{array}$ & 21 & $\begin{array}{l}51,2 \\
51,5\end{array}$ & & & & & & & \\
\hline 20 & $\begin{array}{l}52,5 \\
51,5\end{array}$ & $\begin{array}{l}21 \\
24\end{array}$ & $\begin{array}{l}51,5 \\
51,5\end{array}$ & & & & & & & \\
\hline 21 & $\begin{array}{l}51,5 \\
52,5\end{array}$ & 11 & $\begin{array}{l}51,5 \\
51,9\end{array}$ & & & & & & & \\
\hline $\begin{array}{l}22 \\
23\end{array}$ & $\begin{array}{l}52,5 \\
50,3\end{array}$ & & 51,9 & & & & & & & \\
\hline $\begin{array}{l}23 \\
24\end{array}$ & $\begin{array}{l}50,3 \\
51,5\end{array}$ & $\begin{array}{l}25 \\
16\end{array}$ & 52,2 & & & & & & & \\
\hline 25 & 52,2 & 16 & 52,5 & & & & & & & \\
\hline 26 & 52,8 & 20 & 52,5 & & & & & & & \\
\hline 27 & 44,0 & $\begin{array}{l}22 \\
32\end{array}$ & $\begin{array}{l}52,5 \\
52,5\end{array}$ & & & & & & & \\
\hline 28 & 45,9 & $\begin{array}{r}32 \\
6\end{array}$ & $\begin{array}{l}52,5 \\
52,8\end{array}$ & 53 & 10 & 28 & 1128 & 15,08 & 22,1 & D. \\
\hline $\begin{array}{l}29 \\
30\end{array}$ & $\begin{array}{l}49,0 \\
50,9\end{array}$ & 10 & 52,8 & & & & & & & \\
\hline $\begin{array}{l}30 \\
31\end{array}$ & $\begin{array}{l}50,9 \\
55,0\end{array}$ & 26 & 52,8 & & & & & & & \\
\hline 32 & $\begin{array}{l}55,0 \\
52,5\end{array}$ & $?$ & 53,4 & & & & & & & \\
\hline 33 & $\begin{array}{c}0,0 \\
-\end{array}$ & 5 & 53,7 & & & & & & & \\
\hline 34 & 47,5 & 18 & 54,4 & & & & & & & \\
\hline 35 & 55,0 & 31 & 55,0 & & & & & & & \\
\hline 36 & 55,0 & 35 & 55,0 & 55 & 5 & 14 & 1208 & 16,15 & 21,3 & E. \\
\hline 37 & 49,0 & 36 & 55,0 & & & & & & & \\
\hline 38 & - & 2 & 56,6 & & & & & & & \\
\hline
\end{tabular}


Tabelle VI L. Wandstärke.

\begin{tabular}{|c|c|c|c|c|c|c|c|c|c|c|c|c|c|c|c|c|c|c|c|c|}
\hline \multirow{3}{*}{ No. } & \multicolumn{10}{|c|}{ Links: } & \multicolumn{10}{|c|}{ Rechts: } \\
\hline & \multicolumn{5}{|c|}{ Absolut in $1 / 10 \mathrm{~mm}$} & \multicolumn{5}{|c|}{$\begin{array}{c}\% \text { vom Querschnitt- } \\
\text { Radius }\end{array}$} & \multicolumn{5}{|c|}{ Absolut in $1 / 10 \mathrm{~mm}$} & \multicolumn{5}{|c|}{$\begin{array}{c}\% \text { vom Querschnitt- } \\
\text { Radius }\end{array}$} \\
\hline & $\frac{\pi}{0}$ & 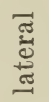 & $\begin{array}{l}\overrightarrow{\tilde{F}} \\
\tilde{0} \\
\tilde{\sigma}\end{array}$ & 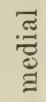 & 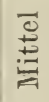 & $\frac{\tilde{\sigma}}{\circ}$ & 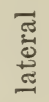 & 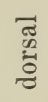 & 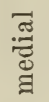 & 㤩 & $\frac{\vec{\sigma}}{0}$ & 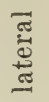 & 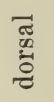 & 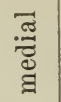 & 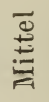 & $\frac{\bar{\pi}}{\circ}$ & 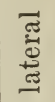 & $\begin{array}{l}\tilde{D} \\
\stackrel{0}{0} \\
\stackrel{0}{0}\end{array}$ & 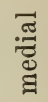 & $\stackrel{\vec{D}}{ \pm}$ \\
\hline
\end{tabular}

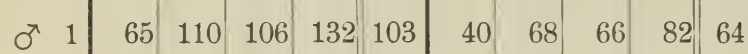
아 2 - $-1-1-$

\begin{tabular}{ll|l|l|l|l|l|l|l|l|l|l|}
\hline & 3 & 57 & 85 & 90 & 97 & 82 & 36 & 52 & 56 & 60 & 51
\end{tabular}

\begin{tabular}{ll|l|l|l|l|l|l|l|l|l|l}
\hline+ & 4 & 55 & 78 & 108 & 112 & 86 & 34 & 50 & 68 & 72 & 56
\end{tabular}

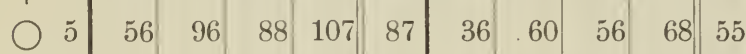

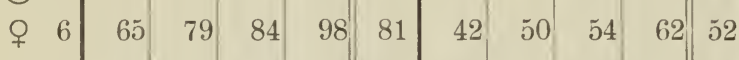

\begin{tabular}{ll|l|l|l|l|l|l|l|l|l|l}
\hline+ & 7 & 80 & 104 & 110 & 140 & 108 & 46 & 60 & 64 & 82 & 63 \\
\hline
\end{tabular}

\begin{tabular}{ll|l|l|l|l|l|l|l|l|l|l}
\hline & 8 & 55 & 76 & 89 & 103 & 81 & 32 & 46 & 54 & 62 & 48
\end{tabular}

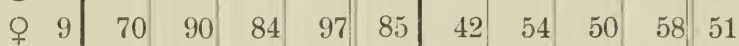

\begin{tabular}{ll|l|l|l|l|l|l|l|l|l|l}
\hline 10 & 65 & 93 & 96 & 117 & 93 & 40 & 58 & 60 & 72 & 57
\end{tabular}

\begin{tabular}{ll|l|l|l|l|l|l|l|l|l|l|}
\hline 11 & 63 & 84 & 82 & 107 & 84 & 40 & 54 & 52 & 68 & 53 \\
\hline
\end{tabular}

\begin{tabular}{ll|l|l|l|l|l|l|l|l|l|l}
\hline 12 & 69 & 92 & 112 & 108 & 95 & 38 & 50 & 60 & 58 & 51 \\
\hline
\end{tabular}

\begin{tabular}{ll|l|l|l|l|l|l|l|l|l|l|}
\hline 13 & 61 & 83 & 113 & 125 & 95 & 36 & 50 & 68 & 76 & 57
\end{tabular}

\begin{tabular}{ll|l|l|l|l|l|l|l|l|l|l}
9 & 14 & 40 & 68 & 100 & 107 & 79 & 26 & 44 & 64 & 68 & 50
\end{tabular}

\begin{tabular}{|l|l|l|l|l|l|l|l|l|l|l|}
15 & 59 & 89 & 79 & 140 & 92 & 36 & 56 & 50 & 86 & 57 \\
\hline
\end{tabular}

\begin{tabular}{l|l|l|l|l|l|l|l|l|l|l|}
\hline 16 & 75 & 92 & 98 & 130 & 99 & 44 & 54 & 58 & 76 & 58 \\
\hline
\end{tabular}

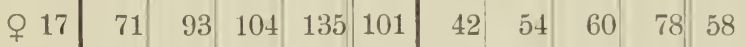

\begin{tabular}{|l|l|l|l|l|l|l|l|l|l|l|}
\hline 18 & 65 & 108 & 98 & 102 & 94 & 38 & 62 & 56 & 58 & 53 \\
\hline
\end{tabular}

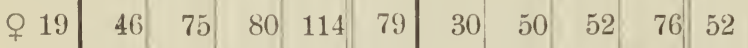

\begin{tabular}{l|l|l|l|l|l|l|l|l|l|l|}
\hline 20 & 59 & 75 & 86 & 115 & 84 & 38 & 48 & 56 & 74 & 54
\end{tabular}

\begin{tabular}{|l|l|l|l|l|l|l|l|l|l|l|}
\hline 21 & 65 & 82 & 90 & 100 & 84 & 46 & 58 & 64 & 72 & 60 \\
\hline
\end{tabular}

\begin{tabular}{|l|l|l|l|l|l|l|l|l|l|l|}
\hline $\mathbf{9} 22$ & 48 & 84 & 90 & 95 & 79 & 32 & 54 & 58 & 62 & 51 \\
\hline
\end{tabular}

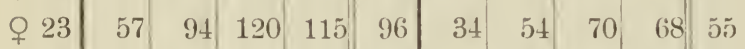

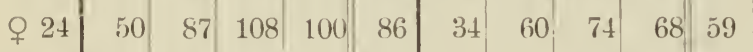

\begin{tabular}{ll|l|l|l|l|l|l|l|l|l|l|}
\hline 225 & 51 & 79 & 105 & 112 & 87 & 32 & 48 & 64 & 68 & 53 \\
\hline
\end{tabular}

\begin{tabular}{ll|l|l|l|l|l|l|l|l|l|l|}
\hline+2 & 26 & 57 & 86 & 100 & 110 & 88 & 38 & 56 & 66 & 72 & 58 \\
\hline
\end{tabular}

\begin{tabular}{|l|l|l|l|l|l|l|l|l|l|l|}
\hline 27 & 70 & 90 & 97 & 103 & 90 & 40 & 52 & 56 & 60 & 52 \\
\hline
\end{tabular}

\begin{tabular}{l|l|l|l|l|l|l|l|l|l|l}
\hline 28 & 58 & 82 & 100 & 118 & 89 & 36 & 50 & 62 & 72 & 55
\end{tabular}

\begin{tabular}{l|l|l|l|l|l|l|l|l|l|l|}
\hline 29 & 56 & 89 & 90 & 110 & 86 & 36 & 58 & 60 & 72 & 56 \\
\hline
\end{tabular}

\begin{tabular}{ll|l|l|l|l|l|l|l|l|l|l|}
\hline 30 & 64 & 98 & 110 & 116 & 97 & 40 & 62 & 70 & 74 & 61 \\
\hline
\end{tabular}

\begin{tabular}{ll|l|l|l|l|l|l|l|l|l|l}
\hline & 31 & 60 & 74 & 100 & 107 & 85 & 36 & 44 & 60 & 64 & 51
\end{tabular}

\begin{tabular}{l|l|l|l|l|l|l|l|l|l|l|}
\hline 332 & 45 & 78 & 100 & 117 & 85 & 30 & 50 & 64 & 76 & 55
\end{tabular}

\begin{tabular}{ll|l|l|l|l|l|l|l|l|l|l|}
\hline 333 & 60 & 72 & 90 & 120 & 85 & 38 & 46 & 56 & 76 & 54 \\
\hline
\end{tabular} \begin{tabular}{llll|l|l|l|l|l|l}
65 & 108 & 100 & 120 & 98 & 40 & 68 & 62 & 76 & 61
\end{tabular} \begin{tabular}{lllll|l|l|l|l|l|l}
60 & 85 & 85 & 98 & 77 & 40 & 56 & 56 & 64 & 53
\end{tabular} \begin{tabular}{l|l|l|l|l|l|l|l|l|l}
67 & 87 & 78 & 98 & 82 & 42 & 54 & 48 & 60 & 51
\end{tabular} \begin{tabular}{|l|l|l|l|l|l|l|l|l|l}
58 & 78 & 105 & 111 & 88 & 36 & 48 & 66 & 70 & 55
\end{tabular} \begin{tabular}{l|l|l|l|l|l|l|l|l|l}
53 & 79 & 75 & 120 & 84 & 32 & 48 & 46 & 74 & 51
\end{tabular} \begin{tabular}{l|l|l|l|l|l|l|l|l|l}
62 & 72 & 86 & 95 & 79 & 38 & 44 & 54 & 60 & 49
\end{tabular} \begin{tabular}{|l|l|l|l|l|l|l|l|l|l|}
80 & 100 & 108 & 140 & 107 & 46 & 58 & 62 & 80 & 61 \\
\hline
\end{tabular}

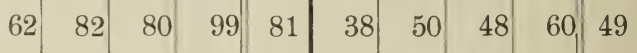
\begin{tabular}{l|l|l|l|l|l|l|l|l|l}
75 & 86 & 86 & 100 & 87 & 46 & 54 & 54 & 62 & 54
\end{tabular} \begin{tabular}{l|l|l|l|l|l|l|l|l|l}
63 & 88 & 86 & 97 & 83 & 40 & 56 & 56 & 62 & 53
\end{tabular} \begin{tabular}{l|l|l|l|l|l|l|l|l|l}
75 & 86 & 87 & 108 & 89 & 46 & 52 & 52 & 66 & 54
\end{tabular} \begin{tabular}{l|l|l|l|l|l|l|l|l|l}
75 & 107 & 104 & 105 & 98 & 40 & 58 & 56 & 56 & 52
\end{tabular}

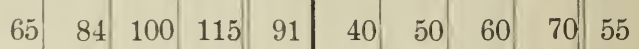
\begin{tabular}{llll|l|l|l|l|l|l}
43 & 66 & 92 & 105 & 76 & 28 & 44 & 60 & 70 & 50
\end{tabular} \begin{tabular}{l|l|l|l|l|l|l|l|l|l}
55 & 95 & 80 & 120 & 87 & 34 & 60 & 50 & 76 & 55
\end{tabular} \begin{tabular}{l|l|l|l|l|l|l|l|l|l}
61 & 90 & 95 & 134 & 95 & 36 & 54 & 56 & 80 & 56
\end{tabular} \begin{tabular}{l|l|l|l|l|l|l|l|l|l}
85 & 132 & 95 & 132 & 111 & 48 & 76 & 54 & 76 & 63
\end{tabular} \begin{tabular}{l|l|l|l|l|l|l|l|l|l}
80 & 100 & 100 & 105 & 96 & 44 & 56 & 56 & 58 & 53
\end{tabular} \begin{tabular}{l|l|l|l|l|l|l|l|l|l}
45 & 79 & 76 & 108 & 77 & 30 & 52 & 50 & 70 & 50
\end{tabular} \begin{tabular}{l|l|l|l|l|l|l|l|l|l}
64 & 86 & 68 & 117 & 84 & 40 & 54 & 44 & 74 & 53
\end{tabular}

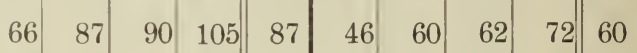
\begin{tabular}{llll|l|l|l|l|l|l}
50 & 79 & 95 & 95 & 80 & 32 & 50 & 60 & 60 & 50
\end{tabular} \begin{tabular}{lll|l|l|l|l|l|l|l}
59 & 89 & 120 & 110 & 94 & 34 & 52 & 70 & 64 & 55
\end{tabular} \begin{tabular}{llll|l|l|l|l|l|l}
56 & 86 & 98 & 110 & 87 & 38 & 58 & 66 & 74 & 59
\end{tabular} \begin{tabular}{l|l|l|l|l|l|l|l|l|l}
49 & 79 & 105 & 108 & 85 & 30 & 48 & 64 & 66 & 52
\end{tabular} \begin{tabular}{l|l|l|l|l|l|l|l|l|l}
56 & 90 & 99 & 104 & 87 & 36 & 58 & 64 & 68 & 56
\end{tabular} \begin{tabular}{l|l|l|l|l|l|l|l|l|l}
68 & 93 & 97 & 105 & 91 & 40 & 56 & 58 & 62 & 54
\end{tabular} \begin{tabular}{lll|l|l|l|l|l|l|l|l}
50 & 99 & 110 & 126 & 96 & 30 & 60 & 68 & 78 & 59
\end{tabular} \begin{tabular}{ll|l|l|l|l|l|l|l|l}
62 & 87 & 88 & 109 & 86 & 40 & 58 & 58 & 72 & 57
\end{tabular} \begin{tabular}{l|l|l|l|l|l|l|l|l|l}
65 & 108 & 105 & 105 & 96 & 40 & 68 & 66 & 66 & 60
\end{tabular} \begin{tabular}{l|l|l|l|l|l|l|l|l|l}
53 & 78 & 97 & 93 & 80 & 32 & 48 & 58 & 56 & 53
\end{tabular} \begin{tabular}{l|l|l|l|l|l|l|l|l|l}
52 & 85 & 95 & 120 & 88 & 32 & 54 & 60 & 76 & 55
\end{tabular} \begin{tabular}{l|l|l|l|l|l|l|l|l|l}
62 & 91 & 90 & 112 & 89 & 40 & 58 & 58 & 72 & 57 \\
\hline
\end{tabular}

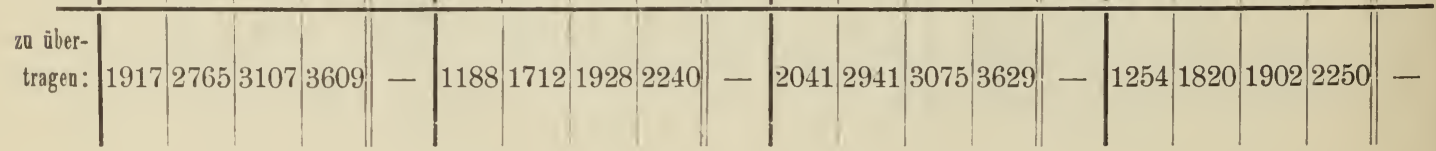


Noch Tabelle VI L.

\begin{tabular}{|c|c|c|c|c|c|c|c|c|c|c|c|c|c|c|c|c|c|c|c|c|}
\hline \multirow{3}{*}{ No. } & \multicolumn{10}{|c|}{ Links: } & \multicolumn{10}{|c|}{ Rechts: } \\
\hline & \multicolumn{5}{|c|}{ Absolut in $1 / 10 \mathrm{~mm}$} & \multicolumn{5}{|c|}{$\begin{array}{c}\% \text { vom Querschnitt- } \\
\text { Radius }\end{array}$} & \multicolumn{5}{|c|}{ Absolut in $1 / 10 \mathrm{~mm}$} & \multicolumn{5}{|c|}{$\begin{array}{c}\% \text { vom Querschnitt- } \\
\text { Radius }\end{array}$} \\
\hline & $\frac{\vec{\sigma}}{0}$ & 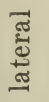 & $\begin{array}{l}\overrightarrow{\widetilde{D}} \\
\tilde{0} \\
\tilde{\Xi} \\
\tilde{O}\end{array}$ & 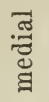 & 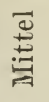 & $\frac{\tilde{\pi}}{0}$ & 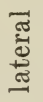 & 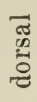 & $\begin{array}{l}\text { 卺 } \\
\text { 品 }\end{array}$ & 䓌 & $\frac{\vec{\sigma}}{0}$ & 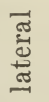 & 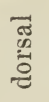 & 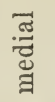 & 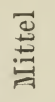 & $\frac{\ddot{\sigma}}{0}$ & 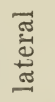 & 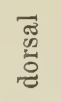 & 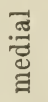 & 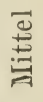 \\
\hline
\end{tabular}

Übertrag : 1917276531073609 -

우 34

우 35

우 36

오 37

우 38

우 39

우 40

ㅇ 41

우 42

우 43

우 44

우 45

오 46

우 47

우 48

우 49

우 50

우 51

우 52

우 53

오 54

오 55

우 56

우 57

우 58

우 59

\begin{tabular}{llll|l}
48 & 72 & 78 & 102
\end{tabular}

\begin{tabular}{l|l|l|l|}
63 & 77 & 83 & 93
\end{tabular}

\begin{tabular}{l|l|l|l|}
68 & 90 & 72 & 107
\end{tabular}

\begin{tabular}{|l|l|l|l|}
67 & 85 & 78 & 117
\end{tabular}

\begin{tabular}{|l|l|l|l|l|}
39 & 75 & 101 & 112
\end{tabular}

\begin{tabular}{|l|l|l|l|}
72 & 77 & 72 & 105
\end{tabular}

\begin{tabular}{l|l|l|l}
85 & 85 & 98 & 105
\end{tabular}

$\begin{array}{llll}57 & 83 & 90 & 80\end{array}$

$\begin{array}{lllll}67 & 85 & 85 & 115\end{array}$

\begin{tabular}{|l|l|l|l|}
61 & 84 & 113 & 125
\end{tabular}

\begin{tabular}{l|l|l|l|}
65 & 88 & 87 & 104
\end{tabular}

\begin{tabular}{ll|l|l|}
52 & 73 & 97 & 103
\end{tabular}

\begin{tabular}{l|l|l|l}
53 & 90 & 103 & 105
\end{tabular}

\begin{tabular}{l|l|l|l|}
67 & 95 & 90 & 110
\end{tabular}

\begin{tabular}{l|l|l|l|}
57 & 94 & 103 & 115
\end{tabular}

\begin{tabular}{lll|l|l|}
55 & 73 & 80 & 113
\end{tabular}

\begin{tabular}{|l|l|l|l|}
43 & 82 & 92 & 125
\end{tabular}

$\begin{array}{lllll}65 & 93 & 100 & 130\end{array}$ \begin{tabular}{llllll|}
78 & 107 & 100 & 117 & 100 \\
\hline
\end{tabular}

$54-\quad-\quad 90-$

\begin{tabular}{|l|r|r|r|r|}
47 & 90 & 100 & 120 & 89 \\
\hline
\end{tabular}

\begin{tabular}{l|l|l|l|l}
54 & 100 & 98 & 95 & 87
\end{tabular}

$7292-107-$

$45 \quad 77-1-$

\begin{tabular}{l|l|l|l|l|}
45 & 75 & 92 & 97 & 77 \\
\hline
\end{tabular}

\begin{tabular}{l|l|l|l|l|}
54 & 83 & 84 & 105 & 81
\end{tabular}

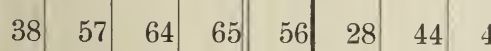

O

운

4862

$42 \quad 54$

$24 \quad 46$

\begin{tabular}{l|l|l|l}
94 & 58 & 6
\end{tabular}

80.32
1188171219282240

2041294130753629

1254182019022250

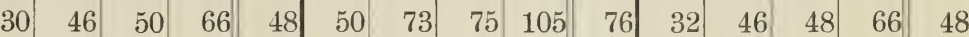

5260

$60 \quad 50$

\begin{tabular}{l|l|l|l|l|}
68 & 78 & 76 & 90 & 78
\end{tabular}

\begin{tabular}{l|l|l|l|l}
42 & 50 & 48 & 56 & 49
\end{tabular}

$\begin{array}{lll}46 & 64 & 60\end{array}$

$74 \quad 55$

$\begin{array}{ll}75 & 91\end{array}$

$67 \quad 91$

$71--$

$70 \quad 60$

$\begin{array}{llll}3 & 93 & 90 & 117\end{array}$

$68 \quad 50$

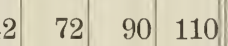

\begin{tabular}{|l|l|l|l|l|l|}
66 & 51 & 72 & 78 & 72 & 105
\end{tabular}

\begin{tabular}{l|l|l|l|l|l|}
62 & 55 & 68 & 81 & 83 & 120
\end{tabular}

$\begin{array}{lll}50 & 50 & 58\end{array}$

$54 \quad 52 \quad 50$

\begin{tabular}{ll|l|l|}
50 & 82 & 85 & 78
\end{tabular}

\begin{tabular}{|l|l|l|l|}
61 & 85 & 82 & 107
\end{tabular}

\begin{tabular}{r|r|r|}
44 & 54 & 54 \\
34 & - & -
\end{tabular}

745

$56-$

\begin{tabular}{|l|l|l|l|}
53 & 75 & 82 & 90
\end{tabular}

$\begin{array}{llll}45 & 83 & 103 & 125\end{array}$

\begin{tabular}{ll|l|l|}
60 & 87 & 94 & 100
\end{tabular}

\begin{tabular}{|l|l|l|l|}
77 & 107 & 98 & 100
\end{tabular}

\begin{tabular}{|l|l|l|l|}
62 & 72 & 93 & 130 \\
\hline
\end{tabular}

\begin{tabular}{l|l|l|l}
63 & 90 & 88 & 100
\end{tabular}

\begin{tabular}{l|l|l|l|}
44 & 72 & 84 & 103
\end{tabular}

$\begin{array}{lllll}42 & 80 & 80 & 102\end{array}$

$50 \quad 62$

\begin{tabular}{llll|l}
42 & 58 & 50 & 70 & 55
\end{tabular}

\begin{tabular}{l|l|l|l|l|l}
931 & 44 & 56 & 54 & 70 & 56
\end{tabular}

\begin{tabular}{l|l|l|l|l|l}
78 & 26 & 44 & 56 & 68 & 48
\end{tabular}

\begin{tabular}{l|l|l|l|l|l}
82 & 46 & 50 & 46 & 66 & 52
\end{tabular}

\begin{tabular}{l|l|l|l|l|l}
88 & 40 & 48 & 50 & 72 & 52
\end{tabular}

\begin{tabular}{l|l|l|l|l|l}
74 & 34 & 56 & 58 & 54 & 50
\end{tabular}

\begin{tabular}{l|l|l|l|l|l}
89 & 40 & 54 & 52 & 70 & 54
\end{tabular}

\begin{tabular}{l|llll|l}
75 & 32 & 46 & 50 & 54 & 45
\end{tabular}

\begin{tabular}{l|l|l|l|l|l}
89 & 28 & 52 & 64 & 78 & 55
\end{tabular}

\begin{tabular}{l|l|l|l|l|l}
85 & 36 & 54 & 58 & 62 & 52
\end{tabular}

\begin{tabular}{l|l|l|l|l|l}
95 & 44 & 62 & 56 & 58 & 55
\end{tabular}

\begin{tabular}{l|l|l|l|l|l}
89 & 38 & 44 & 56 & 78 & 54
\end{tabular}

\begin{tabular}{l|l|l|l|l|l}
85 & 38 & 54 & 52 & 60 & 51
\end{tabular}

\begin{tabular}{lllll|l}
32 & 46 & 60 & 64 & 50
\end{tabular}

$28 \quad 48--$

\begin{tabular}{llllll}
30 & 48 & 60 & 62 & 50 \\
\hline
\end{tabular}

$\begin{array}{llll}50 & 72 & 95 & 97\end{array}$

\begin{tabular}{l|l|l|l|l|l}
76 & 28 & 46 & 52 & 64 & 47
\end{tabular}

\begin{tabular}{l|l|l|l|l|l}
76 & 26 & 50 & 50 & 62 & 47
\end{tabular}

\begin{tabular}{l|l|l|l|l|l|}
88 & 34 & 60 & 68 & 68 & 57
\end{tabular}

\begin{tabular}{l|l|l|l}
62 & 97 & 97 & 101
\end{tabular}

$78 \quad 3$

\begin{tabular}{l|l|l|l|l}
32 & 48 & 62 & 64 & 51
\end{tabular}

\begin{tabular}{l|l|l|l}
40 & 56 & 54
\end{tabular}

$66 \quad 54$

\begin{tabular}{l|l|l|l|}
80 & 100 & 87 & 110
\end{tabular}

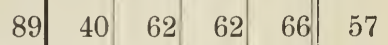

$68 \quad 55$

$66 \quad 47$

$\begin{array}{lllll}47 & 95 & 100 & 117\end{array}$

\begin{tabular}{ll|l|l|l}
55 & 80 & 83 & 110
\end{tabular}

\begin{tabular}{l|l|l}
94 & 46 & 58
\end{tabular}

52

$64 \quad 55$

\begin{tabular}{l|llll|l}
88 & 28 & 58 & 60 & 72 & 54
\end{tabular}

\begin{tabular}{|l|l|l|l|l|}
26 & 50 & 56 & 76 & 52 \\
\hline
\end{tabular}

\begin{tabular}{l|ll|l|l|l|l|l|l|l}
50 & 60 & 88 & 132 & 82 & 30 & 36 & 54 & 82 & 50
\end{tabular}

\begin{tabular}{l|ll|l|l|l|l|l|l|l}
65 & 96 & 105 & 135 & 100 & 36 & 54 & 60 & 76 & 55
\end{tabular}

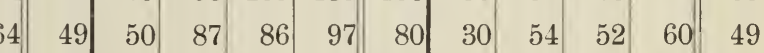
\begin{tabular}{l|l|l|l|l|}
70 & 87 & 90 & 127 & 93 \\
\hline
\end{tabular}

62

\begin{tabular}{l|l|l|l|l|}
70 & 87 & 75 & 122 & 88 \\
\hline
\end{tabular} \begin{tabular}{l|l|l|l|l}
46 & 58 & 50 & 80 & 58
\end{tabular}

Samme: $34504890|52036306|$ je

- 2138 3028 $3232|3910|$

- 3554511853396320 - 2194316833003906 -

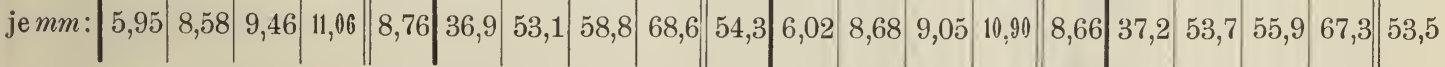

\begin{tabular}{ll|llll|l|l|l|l|l|l|l} 
Durchschnitt von rechts und links zusammen $m m:$ & 5,99 & 8,63 & 9,25 & 10,98 & 8,71 & 37,0 & 53,4 & 57,3 & 68,0 & 53,9
\end{tabular} 
Tabelle VI S. Wandstärke.

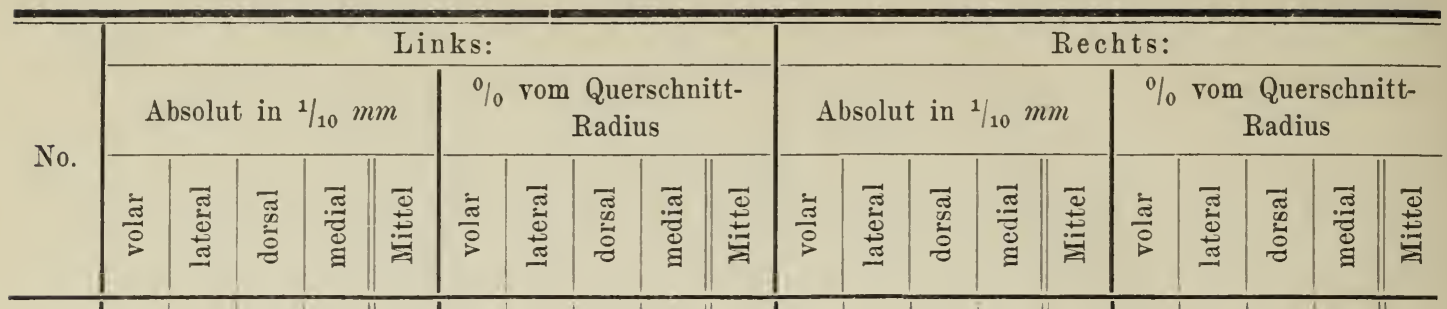

\begin{tabular}{|ll|l|l|l|l|l|l|l|l|l|l|}
\hline & 1 & 70 & 85 & 78 & 98 & 83 & 44 & 52 & 48 & 60 & 51 \\
\hline
\end{tabular}

\& 3

○ 4

5

우 6

우 7

우 9

$\bigcirc 10$

우 11

우 12

13

14

15

$\bigcirc 16$

우 17

$\bigcirc 18$

$\bigcirc 19$

$\bigcirc 20$

ㅇ 21

우 22

○ 23

$\bigcirc 2$

$\bigcirc 26$

$\bigcirc 27$

$\bigcirc 28$

우 29

$\bigcirc 30$

ㅇ 31

우 32

$\bigcirc 3$

우 34

$\bigcirc 35$

우 36

우 37

38
26

$\begin{array}{lll}42 & 46 & 38\end{array}$

\begin{tabular}{l|l|l|}
38 & 50 & 54
\end{tabular}

\begin{tabular}{ll|l}
28 & 38 & 42
\end{tabular}

\begin{tabular}{l|l|l}
36 & 54 & 52
\end{tabular}

\begin{tabular}{l|l|l}
34 & 48 & 54
\end{tabular}

\begin{tabular}{l|l|l|l|}
88 & 26 & 42 & 50
\end{tabular}

\begin{tabular}{ll|l}
30 & 38 & 50
\end{tabular}

\begin{tabular}{l|l|}
26 & 46
\end{tabular}

\begin{tabular}{llll|l}
48 & 52 & 46 & - & -
\end{tabular}

$42 \quad 54$

$\begin{array}{lll}36 & 46 & 52\end{array}$

6251

$\begin{array}{llll}76 & 44 & 54 & 52\end{array}$ \begin{tabular}{|ll|r|r|r|r|r}
\hline+ & 2 & 71 & 107 & 116 & 132 & 106 \\
\hline
\end{tabular}

$\bigcirc 8$

$\bigcirc 25$ \begin{tabular}{|l|r|r|r|r|r}
50 & 87 & 90 & 116 & 86 \\
\hline
\end{tabular}

\begin{tabular}{l|lll|}
86 & 97 & 80 & 135
\end{tabular}

\begin{tabular}{l|l|l|l}
68 & 92 & 100 & 122
\end{tabular}

\begin{tabular}{r|r|r|r|}
52 & 70 & 78 & 95
\end{tabular}

\begin{tabular}{r|r|r|r|r|}
48 & 88 & 97 & 128 & 90 \\
\hline
\end{tabular}

$\begin{array}{rrrrrr}68 & 102 & 98 & 150 & 104\end{array}$

\begin{tabular}{l|l|l|l}
66 & 92 & 106 & 120
\end{tabular}

\begin{tabular}{l|r|r|r|}
51 & 80 & 95 & 126
\end{tabular}

\begin{tabular}{l|l|l|l|}
56 & 73 & 95 & 123
\end{tabular}

\begin{tabular}{|l|l|l|l|l|}
45 & 78 & 96 & 104
\end{tabular}

\begin{tabular}{lrrr|r|r}
93 & 100 & 87 & - & -
\end{tabular}

\begin{tabular}{ll|l|l|}
76 & 97 & 83 & 111
\end{tabular}

$\begin{array}{lllll}50 & 95 & 105 & 120\end{array}$

\begin{tabular}{ll|l|l|}
65 & 97 & 96 & 120
\end{tabular}

\begin{tabular}{l|l|l|l|}
64 & 84 & 95 & 119
\end{tabular}

\begin{tabular}{lll|l|l}
71 & 95 & 93 & 136
\end{tabular}

\begin{tabular}{l|l|l|}
70 & $93-103$
\end{tabular}

\begin{tabular}{l|l|l|l}
56 & 95 & 90 & 102
\end{tabular}

\begin{tabular}{l|l|l|l|}
72 & 106 & 95 & 122
\end{tabular}

\begin{tabular}{|l|l|l|l|}
47 & 80 & 87 & 100
\end{tabular}

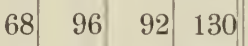

$\begin{array}{llll}64 & 95 & 82 & 130\end{array}$

\begin{tabular}{l|l|l|l|}
67 & 88 & 92 & 123
\end{tabular}

$6292-105-$

\begin{tabular}{l|l|l|l}
65 & 92 & 73 & 102
\end{tabular}

\begin{tabular}{llll|}
62 & 90 & 75 & 107
\end{tabular}

$\begin{array}{lllll}55 & 92 & 93 & 130\end{array}$

$\begin{array}{llll}60 & 83 & 78 & 105\end{array}$

$\begin{array}{lllll}50 & 80 & 95 & 110\end{array}$

\begin{tabular}{l|l|l|l|}
65 & 100 & 88 & 135
\end{tabular}

$\begin{array}{lllll}80 & 100 & 94 & 130\end{array}$

\begin{tabular}{l|l|l|l}
75 & 95 & 88 & 126
\end{tabular}

\begin{tabular}{l|lll|}
50 & 90 & 105 & 108
\end{tabular}

\begin{tabular}{l|l|l|l}
56 & 88 & 98 & 120
\end{tabular}

\begin{tabular}{l|l|l|l|}
80 & 98 & 87 & 127
\end{tabular}

92

0

99

83

\begin{tabular}{l|l|l|l|l|}
48 & 85 & 112 & 147 & 103
\end{tabular}

\begin{tabular}{l|l|l|l|l}
72 & 97 & 80 & 100 & 87
\end{tabular}

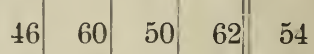
$\bigcirc 38$ \begin{tabular}{l|l|l}
34 & 52 & 52
\end{tabular}

\begin{tabular}{|l|l|l|}
34 & 46 & 46
\end{tabular}

\begin{tabular}{l|ll}
38 & 50 & -
\end{tabular}

\begin{tabular}{l|l|l|l}
86 & 32 & 54 & 52 \\
99 & 40 & 60 & 54
\end{tabular}

\begin{tabular}{l|l|l|l|}
78 & 26 & 44 & 48
\end{tabular}

\begin{tabular}{l|l|l|l|}
96 & 36 & 50 & 48
\end{tabular}

\begin{tabular}{ll|l|l}
93 & 34 & 50 & 42
\end{tabular}

\begin{tabular}{l|l}
36 & 46
\end{tabular}

\begin{tabular}{l|l|l|}
26 & 46 & 60
\end{tabular}

$3856-$

92

\begin{tabular}{l|l|l|l}
81 & 34 & 46 & 44
\end{tabular}

$\begin{array}{llll}84 & 30 & 46 & 56\end{array}$

97 34

\begin{tabular}{ll|l|l}
96 & 40 & 50 & 48
\end{tabular}

\begin{tabular}{l|l|l|l|}
88 & 26 & 46 & 54
\end{tabular}

$\begin{array}{llll}90 & 32 & 50 & 54\end{array}$

$64 \quad 50$

$66 \quad 50 \quad 63$

\begin{tabular}{l|l|l}
66 & 48
\end{tabular}

$56-$

$58 \quad 49$

$\begin{array}{rrrr}47 & 85 & 73 & 104\end{array}$

\begin{tabular}{rr|r|r|r|r}
54 & 13 & 60 & 84 & 93 & 122
\end{tabular}

\begin{tabular}{ll|l|l|l|l|}
68 & 50 & 65 & 95 & 90 & 142
\end{tabular}

\begin{tabular}{l|l|l|l|l|l}
68 & 48 & 67 & 105 & 80 & 115
\end{tabular}

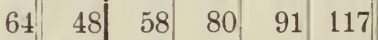

\begin{tabular}{l|l|l|l|l|l|}
78 & 52 & 48 & 85 & 107 & 143
\end{tabular}

\begin{tabular}{|l|l|l|l|l|l|}
64 & - & 69 & 88 & 88 & 118
\end{tabular}

\begin{tabular}{l|l|l|l|}
69 & 88 & 88 & 118 \\
69 & 82 & 68 & 110
\end{tabular}

$\begin{array}{lllllll}58 & 45 & 56 & 95 & 80 & 110\end{array}$

\begin{tabular}{l|l|l|l|l|l}
74 & 52 & 54 & 100 & 90 & 132
\end{tabular}

$\begin{array}{llllll}60 & 46 & 60 & 90 & 80 & 105\end{array}$

\begin{tabular}{l|l|l|l|l|l}
64 & 49 & 55 & 88 & 95 & 112
\end{tabular}

\begin{tabular}{l|l|l|l|l|l|}
70 & 50 & 63 & 95 & 90 & 137
\end{tabular}

72

72
68

56

\begin{tabular}{r|r|r|r|r}
45 & 76 & 100 & - & 125 \\
51 & 75 & 93 & 88 & 130
\end{tabular}

\begin{tabular}{l|r|r|rr|}
45 & 51 & 96 & 105 & 105
\end{tabular}

\begin{tabular}{|r|r|r|r|r|r|}
\hline 66 & 50 & 55 & 93 & 94 & 118 \\
66 & 51 & 73 & 97 & 78 & 120 \\
\hline
\end{tabular} \begin{tabular}{|l|l|l|l|l}
34 & 54 & 62 & 70 & 55
\end{tabular} \begin{tabular}{|l|l|l|l|l}
28 & 44 & 48 & 54 & 43
\end{tabular} \begin{tabular}{|l|l|l|l|l}
48 & 54 & 38 & 66 & 51
\end{tabular} \begin{tabular}{l|l|l|l|l}
38 & 50 & 48 & 62 & 49
\end{tabular} \begin{tabular}{l|l|l|l|l}
24 & 40 & 38 & 44 & 36
\end{tabular} \begin{tabular}{lllll|l}
28 & 48 & 50 & 70 & 49
\end{tabular}

Samme: $|2403345733124427||1304186817562384-| 2378346932524493|-| 1262183417222378||-$

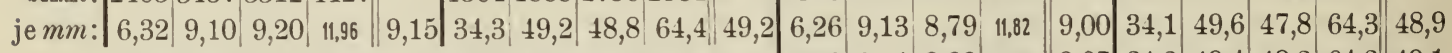

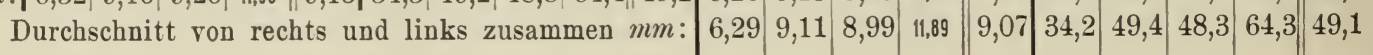


Zu Tabelle VI. Spezialtabelle über die mittlere Wandstärke der Wallache.

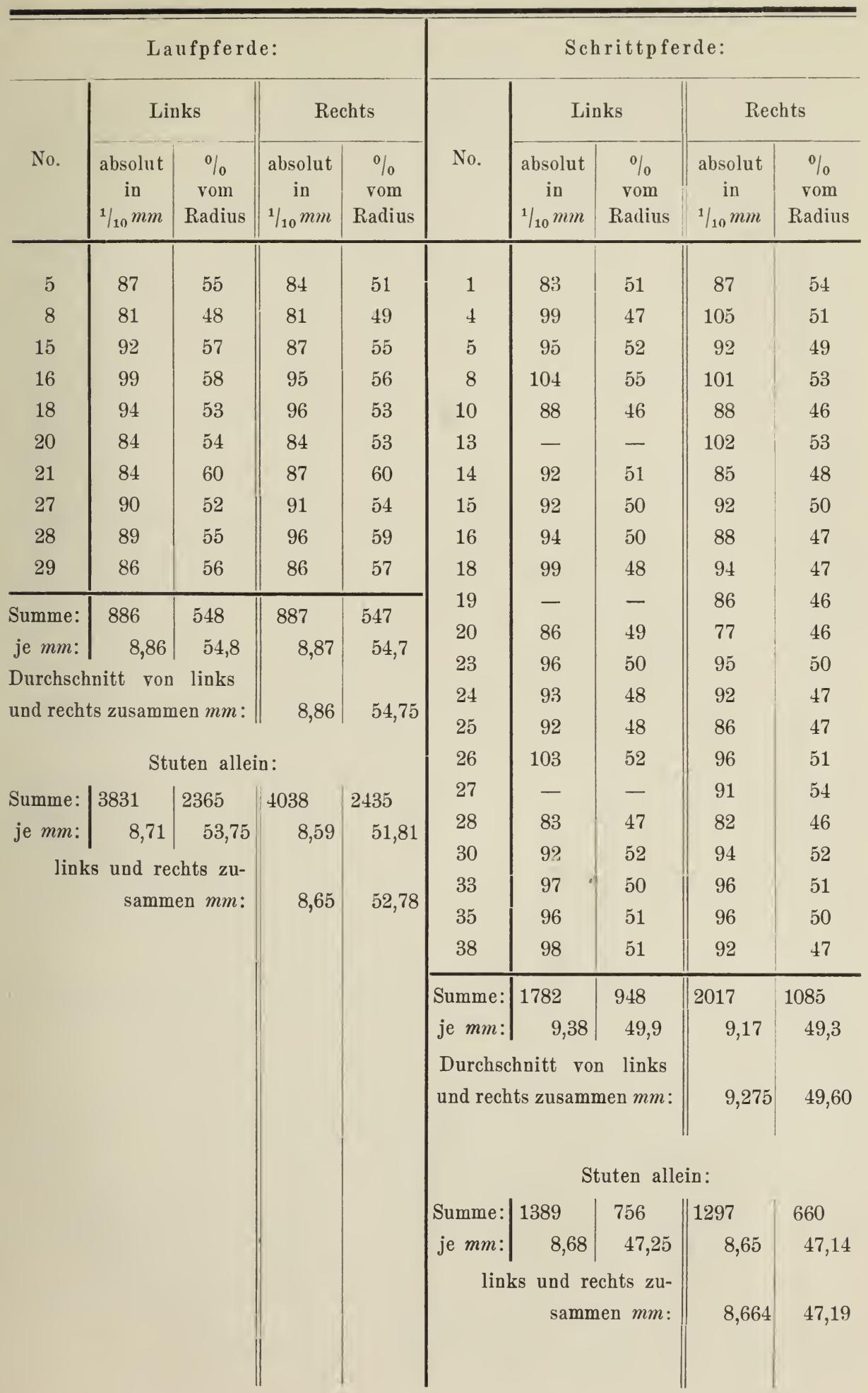


Tabelle VII. Zusammenstellung der RöhrbeinmaIse.

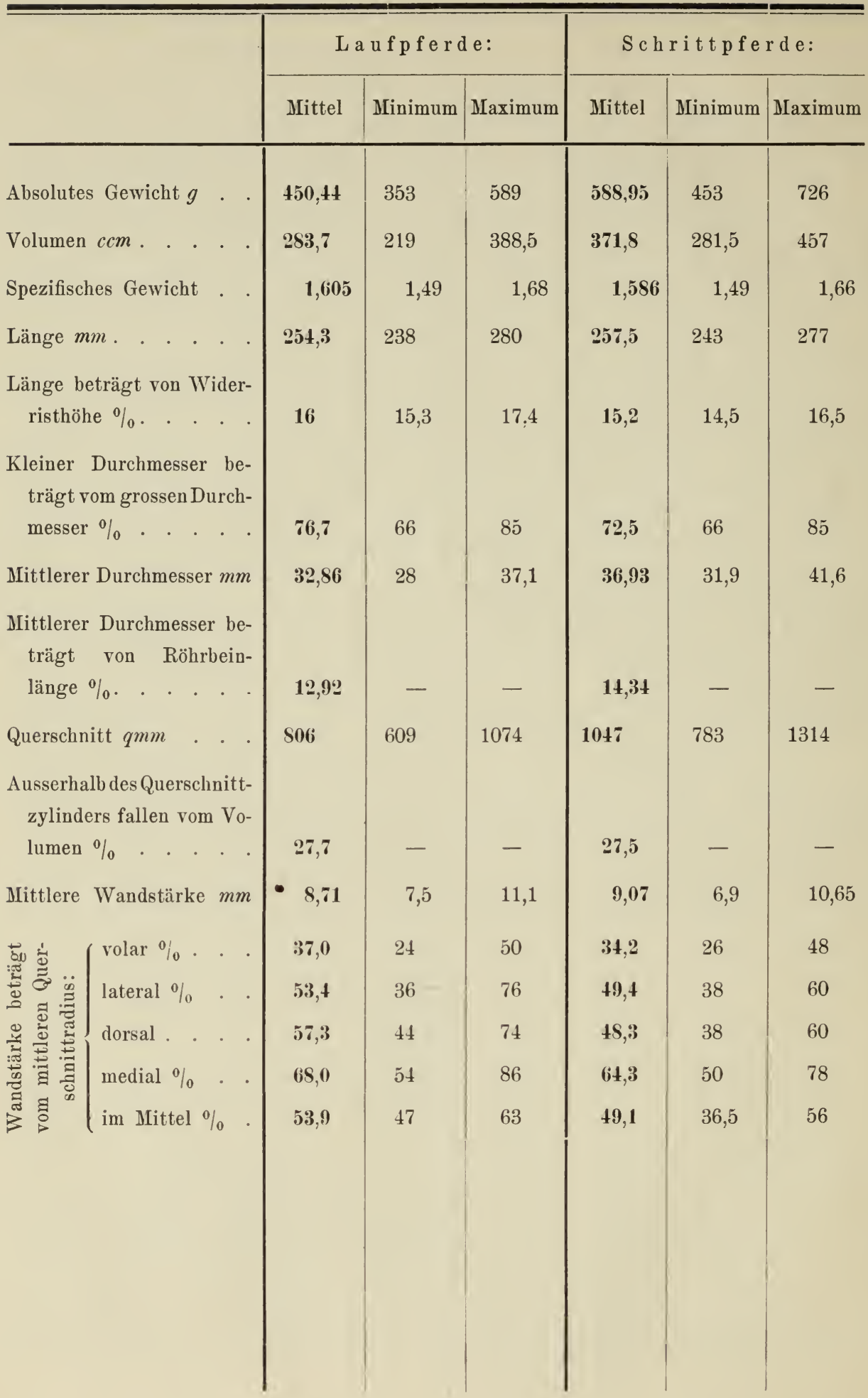


Tabelle VIII. Asymmetrie der Röhrbeinpaare.

\begin{tabular}{|c|c|c|c|c|c|c|c|c|}
\hline & \multicolumn{4}{|c|}{ Laufpferde: } & \multicolumn{4}{|c|}{ Schrittpferde: } \\
\hline & \multicolumn{2}{|c|}{$\begin{array}{l}\text { Der Durch- } \\
\text { schnitt be- } \\
\text { trägt für das }\end{array}$} & \multicolumn{2}{|c|}{\begin{tabular}{|c|} 
Grösste \\
Differenz zu- \\
gunsten des
\end{tabular}} & \multicolumn{2}{|c|}{$\begin{array}{l}\text { Der Durch- } \\
\text { schnitt be- } \\
\text { trägt für das }\end{array}$} & \multicolumn{2}{|c|}{\begin{tabular}{||c} 
Grösste \\
Differenz zu- \\
gunsten des
\end{tabular}} \\
\hline & linke & rechte & $\begin{array}{c}\text { linken } \\
\%\end{array}$ & $\begin{array}{c}\text { rechten } \\
\%\end{array}$ & linke & rechte & $\begin{array}{c}\text { linken } \\
\%\end{array}$ & $\begin{array}{c}\text { rechten } \\
\%\end{array}$ \\
\hline 1. Absolutes Gewicht $g$. & 450,7 & 450,2 & 6,5 & - & 588,8 & 589,2 & 6,6 & - \\
\hline No. der betr. Knochen . & - & - & L 38 & - & - & - & S 34 & - \\
\hline 2. Volumen $\mathrm{ccm}$. . . . & 283,7 & 283,8 & 7,1 & - & 370,8 & 372,8 & 5,9 & - \\
\hline No. der betr. Knochen. & - & - & L 30 & - & - & - & S 34 & - \\
\hline 3. Spez. Gewicht. . . . & 1,605 & 1,605 & - & 7,3 & 1,588 & 1,585 & - & 4,6 \\
\hline No. der betr. Knochen . & - & - & - & L 34 & - & - & - & S 7 \\
\hline 4. Länge $\mathrm{mm}$. . . . . & 254,3 & 2554,3 & 1,2 & - & 257,1 & 257,9 & - & 1,6 \\
\hline No. der betr. Knochen. . & - & - & L 13 & - & - & - & - & S 17 \\
\hline $\begin{array}{l}\text { 5. Länge beträgt von Wider- } \\
\text { risthöhe } \%\end{array}$ & 16 & 16 & 1,3 & - & 15,2 & 15,2 & - & 1,3 \\
\hline No. der betr. Knochen. . & - & - & L 13 & - & - & - & - & S 17 \\
\hline $\begin{array}{l}\text { 6. Kleiner Durchmesser be- } \\
\text { trägt vom grossen } \%\end{array}$ & 76,57 & 76,91 & 10 & - & 72,39 & 72,70 & - & 16 \\
\hline No. der betr. Knochen. . & - & - & L 5 & - & - & - & - & S 12 \\
\hline 7. Mittl. Durchmesser $\mathrm{mm}$ & 32,22 & 32,38 & - & 4,9 & 36,97 & 36,8 こ & - & 6,5 \\
\hline No. der betr. Knochen. . & - & - & - & L 47 & - & - & - & S 12 \\
\hline $\begin{array}{l}\text { 8. Mittl. Durchmesser beträgt } \\
\text { von Röhrbeinlänge } \% \text {. }\end{array}$ & 12,68 & 12,73 & - & - & 14,38 & 14,34 & - & - \\
\hline 9. Querschn & 821,81 & 832,66 & - & 10 & 1050,16 & 1045,70 & - & 15 \\
\hline No. der betr. Knochen. . & - & - & - & L 47 & - & - & - & S 12 \\
\hline $\begin{array}{l}\text { 10. Ausserhalb des Querschnitt- } \\
\text { zylinders fallen Volumen } \%\end{array}$ & 28,2 & 27,2 & - & - & 27,2 & 27,7 & - & - \\
\hline 11. Mittl. Wandstärke $\mathrm{mm}$. & 8,76 & 8,66 & $\left.18^{1}\right)$ & - & 9,15 & 9 & 10 & - \\
\hline No. der betr. Knochen. . & - & - & - & - & - & - & S 20 & - \\
\hline $\begin{array}{l}\text { 12. Mittl. Wandstärke beträgt } \\
\text { vom mittl. Radius } \% \text {. } \\
\text { No. der betr. Knochen. }\end{array}$ & $\begin{array}{r}\tilde{5} 4,3 \\
-\end{array}$ & $\begin{array}{c}53,5 \\
-\end{array}$ & - & $\begin{array}{c}10 \\
\text { L } 17\end{array}$ & $\begin{array}{l}49,2 \\
-\end{array}$ & $\begin{array}{c}48,9 \\
-\end{array}$ & $\begin{array}{l}- \\
-\end{array}$ & $\begin{array}{c}8 \\
\text { S } 4\end{array}$ \\
\hline
\end{tabular}

Tabelle IX. Tragkraft.

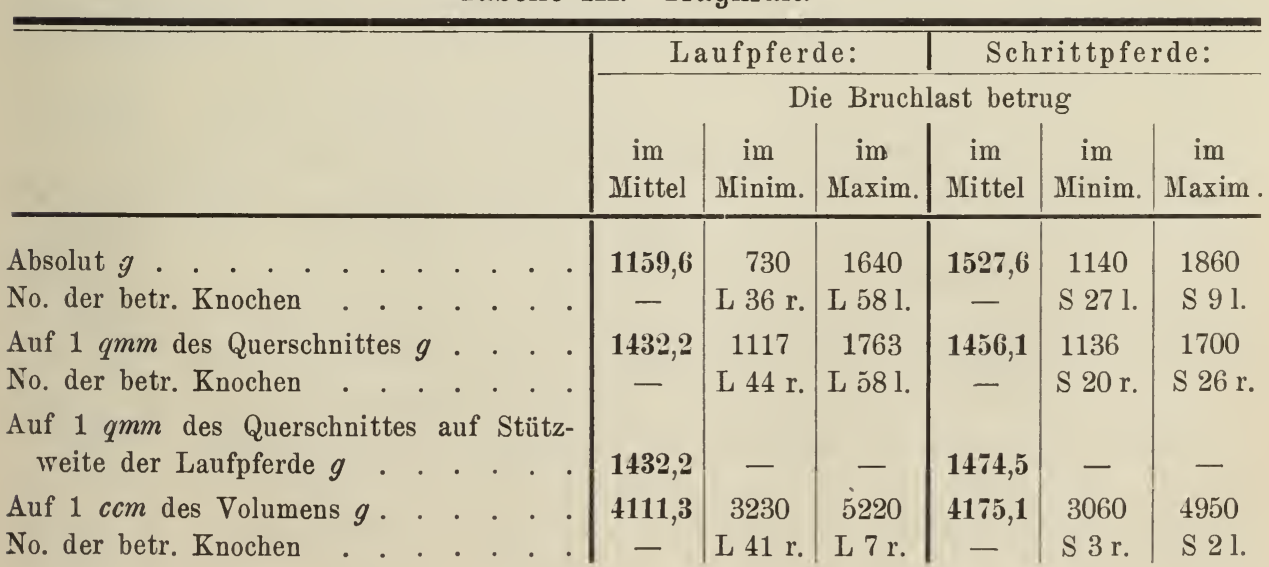

1) Verworfen. 
Tabelle X. Durchbiegung.

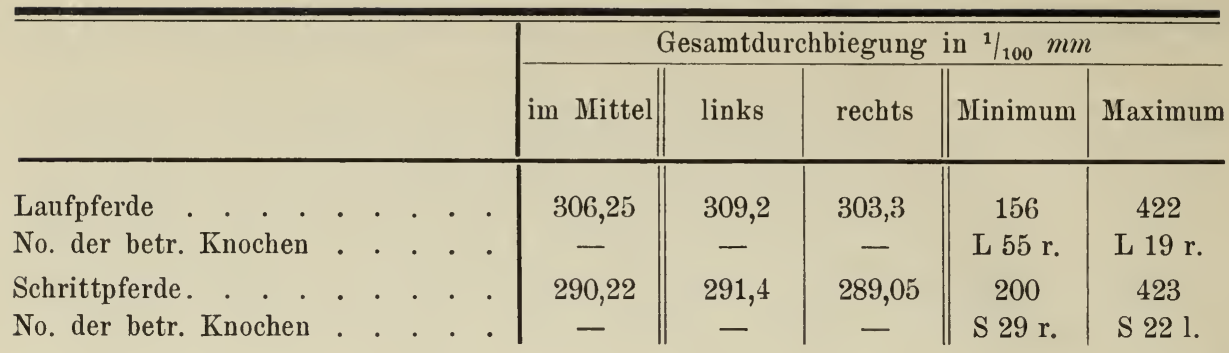

Tabelle XI. Asymmetrie der Leistung.

\begin{tabular}{|c|c|c|c|c|c|c|c|c|}
\hline & \multicolumn{4}{|c|}{ Laufpferde: } & \multicolumn{4}{|c|}{ Schrittpferde: } \\
\hline & \multicolumn{2}{|c|}{ Durchschnitt } & \multicolumn{2}{|c|}{$\begin{array}{l}\text { Grösste } \\
\text { Differenz }\end{array}$} & \multicolumn{2}{|c|}{ Durchschnitt } & \multicolumn{2}{|c|}{$\begin{array}{l}\text { Grösste } \\
\text { Differenz }\end{array}$} \\
\hline & links & rechts & $\begin{array}{c}\text { links } \\
\% \\
\end{array}$ & $\begin{array}{c}\text { rechts } \\
\%\end{array}$ & links & rechts & $\begin{array}{c}\text { links } \\
\% \\
\end{array}$ & $\begin{array}{c}\text { rechts } \\
\% \\
\end{array}$ \\
\hline Bruchlast absolut $\mathrm{kg}$. & 1164,8 & 1154,4 & 20 & 11 & 1536,6 & 1518,6 & 18 & 21 \\
\hline No. der betr. Knochen . & - & - & L 5 & L 24 & - & - & S 7 & S 26 \\
\hline $\begin{array}{l}\text { Bruchlast auf } 1 q m m \text { des Quer- } \\
\text { schnittes } g \text {. . . . . }\end{array}$ & 1446,6 & 1417,9 & - & - & 1464,6 & 1447,7 & - & 一 \\
\hline $\begin{array}{l}\text { Bruchlast auf } 1 \mathrm{gmm} \text { des Quer- } \\
\text { schnittes und auf gleiche } \\
\text { Stützweite (der Laufpferde) } g\end{array}$ & 1446,6 & 1417,9 & - & - & 1483,03 & 1465,91 & - & - \\
\hline $\begin{array}{c}\text { Bruchlast auf } 1 \mathrm{ccm} \text { des Vo- } \\
\text { lumens } g . \\
\end{array}$ & 4131,0 & 4091,5 & - & - & 4204,0 & 4146,0 & - & - \\
\hline $\begin{array}{l}\text { Gesamtdurchbiegung der } 2 . \\
\text { Hälfte } 1 / 100 \mathrm{~mm} .{ }^{2} \text {. . . }\end{array}$ & 309,2 & 303,3 & 26 & 36 & 291,4 & 289,05 & 32 & 29 \\
\hline No. der betr. Knochen . . & - & 1 - & L 37 & L 57 & 1 - & - & S 22 & S 33 \\
\hline
\end{tabular}

Tabelle XII. Leistungsfähigkeit nur der stärkeren Röhrbeine der einzelnen Paare.

\begin{tabular}{|c|c|c|c|c|}
\hline & \multicolumn{2}{|c|}{$\begin{array}{l}\text { Durchschnittliche } \\
\text { Leistungsfähigkeit: }\end{array}$} & \multicolumn{2}{|c|}{$\begin{array}{c}\text { Leistungsfähigkeit } \\
\text { uur des stärkeren } \\
\text { Röhrbeins: }\end{array}$} \\
\hline & $\begin{array}{l}\text { Lauf- } \\
\text { pferde }\end{array}$ & $\begin{array}{l}\text { Schritt- } \\
\text { pferde }\end{array}$ & $\begin{array}{l}\text { Lauf- } \\
\text { pferde }\end{array}$ & $\begin{array}{l}\text { Schritt- } \\
\text { pferde }\end{array}$ \\
\hline Absolute Bruchlast $\mathrm{kg}$. & $\begin{array}{c}1159,6 \\
-\end{array}$ & $\begin{array}{c}1527,6 \\
+31,7 \%\end{array}$ & $\begin{array}{c}1184,6 \\
-\end{array}$ & $\begin{array}{r}1571,3 \\
+32,7 \%\end{array}$ \\
\hline Bruchlast für je $1 \mathrm{qmm}$ des Querschnittes $g$ & $\begin{array}{c}1432,25 \\
-\end{array}$ & $\begin{array}{r}1456,15 \\
+1,67 \%\end{array}$ & $\begin{array}{c}1468,0 \\
-\end{array}$ & $\begin{array}{r}1492,3 \\
+1,63 \%\end{array}$ \\
\hline Bruchlast für je $1 q m m$ bei gleicher Stützweite $g$ & $\begin{array}{c}1432,25 \\
-\end{array}$ & $\begin{array}{r}1474,47 \\
+2,94 \%\end{array}$ & $\begin{array}{c}1468,0 \\
-\end{array}$ & $\begin{array}{c}1511,1 \\
+2,93 \%\end{array}$ \\
\hline Bruchlast für je $1 \mathrm{ccm}$ des Volumens $g$ & $\begin{array}{c}4111,3 \\
-\end{array}$ & $\begin{array}{c}4175,1 \\
+1,56 \%\end{array}$ & $\begin{array}{c}4203,0 \\
-\end{array}$ & $\begin{array}{r}4226,0 \\
+0,55 \%\end{array}$ \\
\hline Absolute Durchbiegung $1 / 100 \mathrm{~mm}$. & $\begin{array}{c}306,25 \\
-\end{array}$ & $\begin{array}{r}290,22 \\
-5,53 \%\end{array}$ & $\begin{array}{c}317,2 \\
-\end{array}$ & $\begin{array}{c}302,6 \\
-4,86 \%\end{array}$ \\
\hline
\end{tabular}




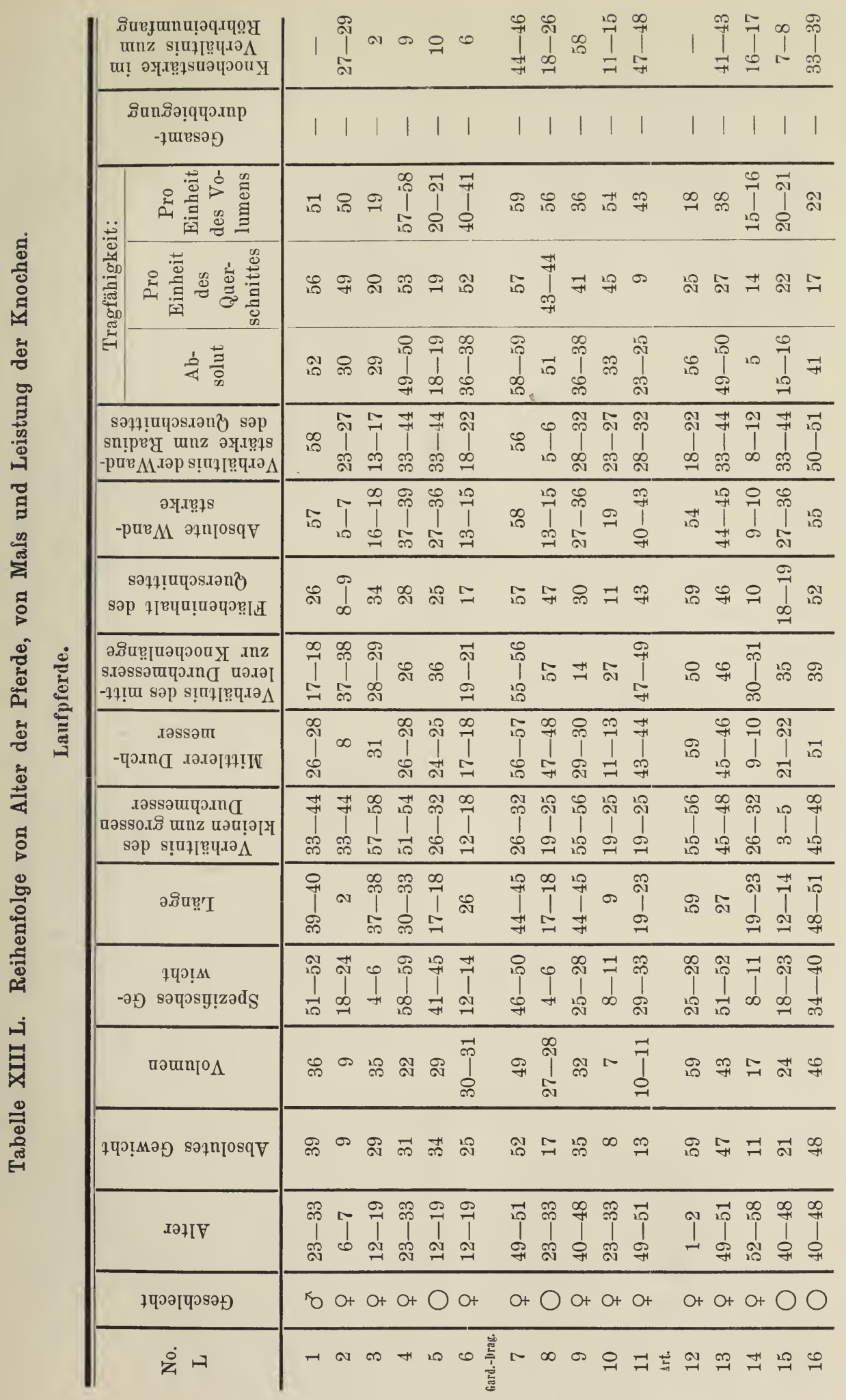




\begin{tabular}{|c|c|}
\hline 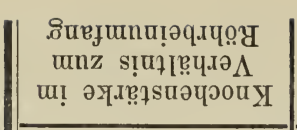 & 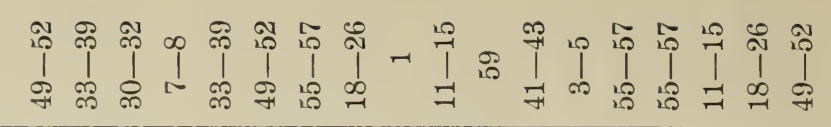 \\
\hline 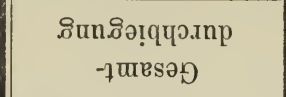 & | డై \\
\hline 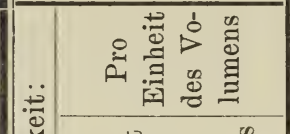 & $\exists$ ง \\
\hline 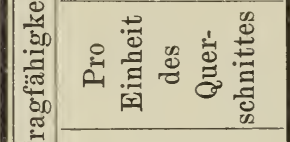 & 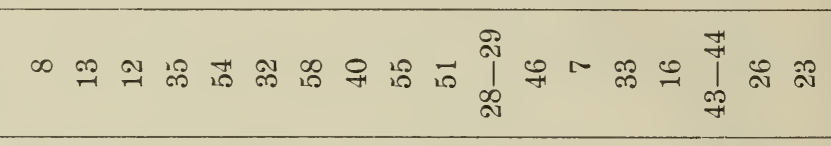 \\
\hline 完荳 & 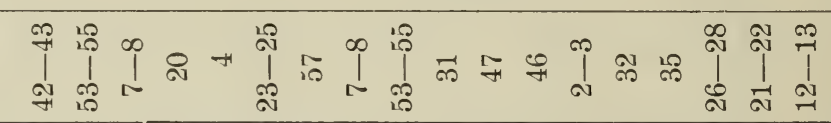 \\
\hline 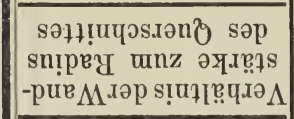 & 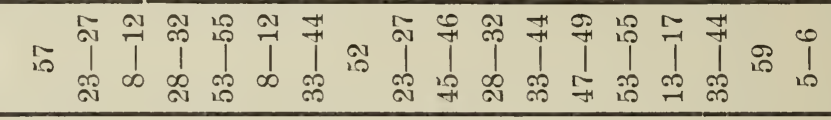 \\
\hline 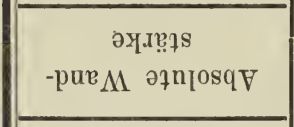 & 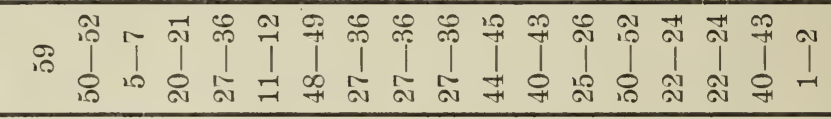 \\
\hline 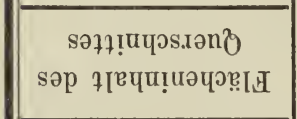 & 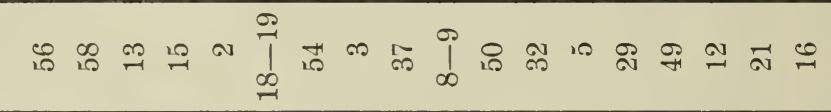 \\
\hline 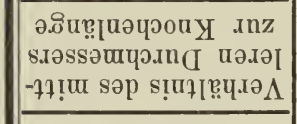 & 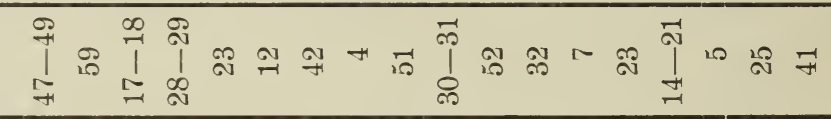 \\
\hline 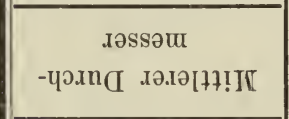 & 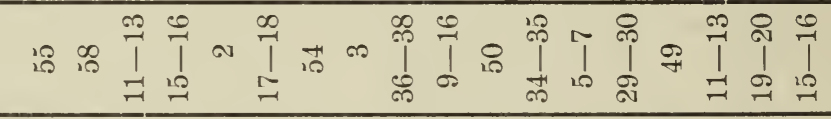 \\
\hline 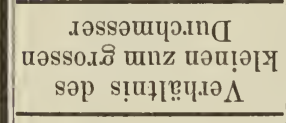 & 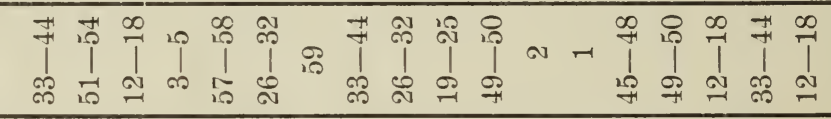 \\
\hline ว.ภิuẹT & 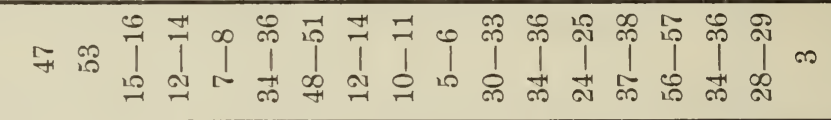 \\
\hline 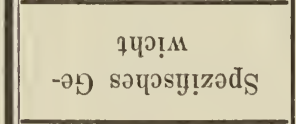 & 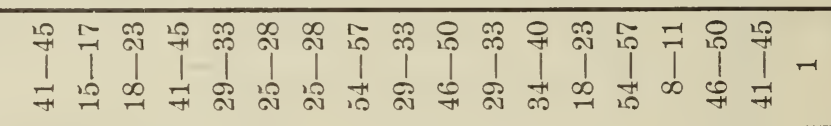 \\
\hline иәшпю ${ }^{\circ} \Lambda$ & 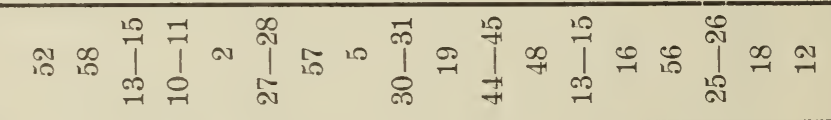 \\
\hline 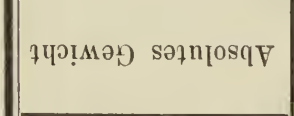 & 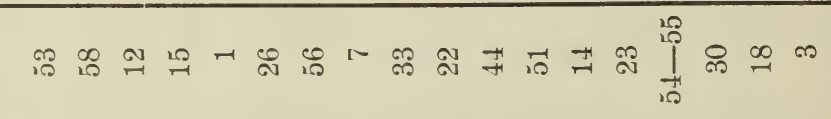 \\
\hline .әશІV & 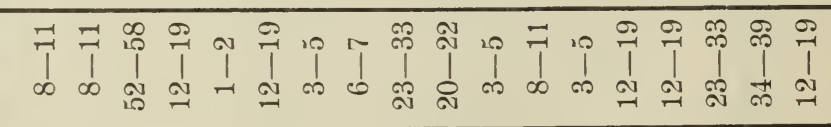 \\
\hline 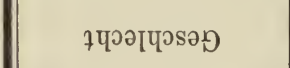 & 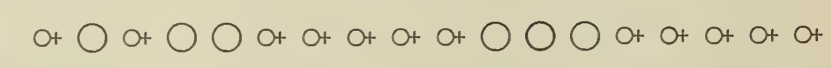 \\
\hline & $=$ \\
\hline
\end{tabular}




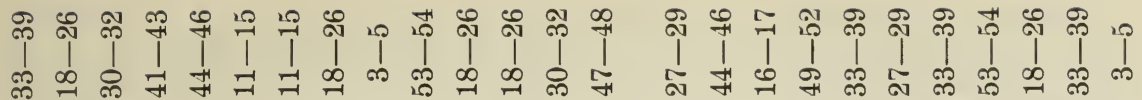

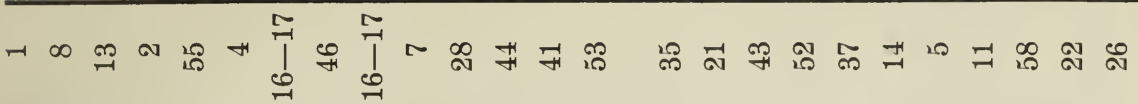

ล9

당

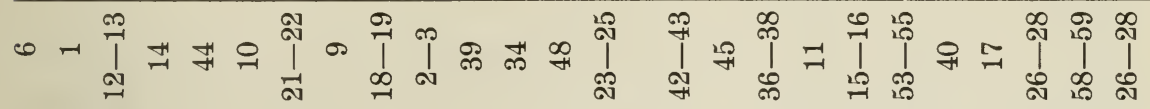

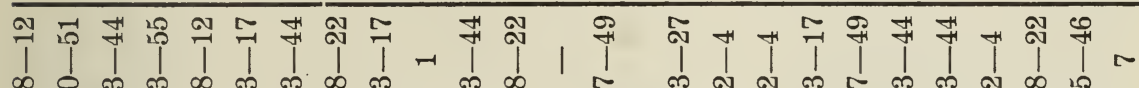
$\infty$ ది

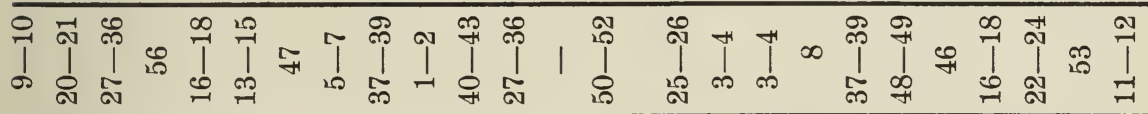

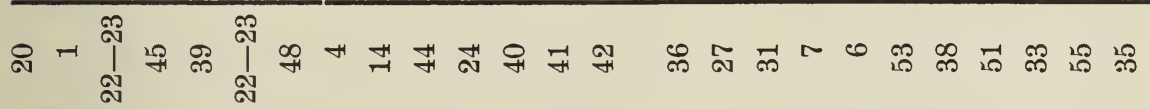

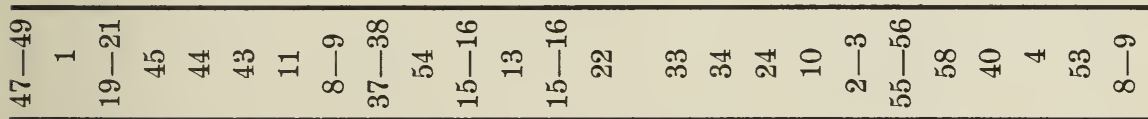

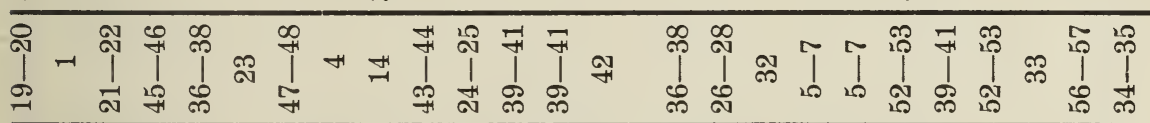

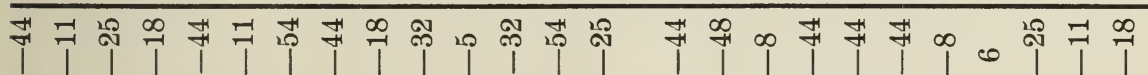

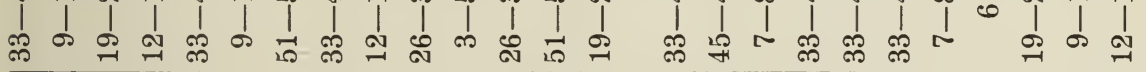

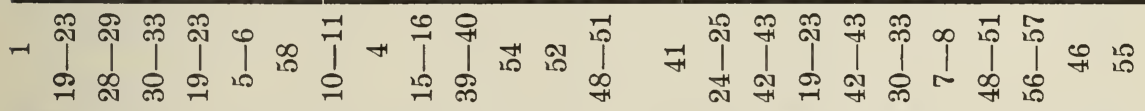

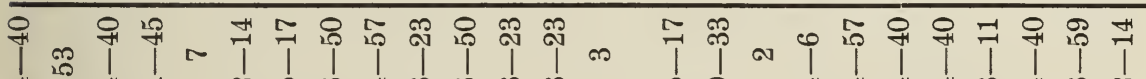

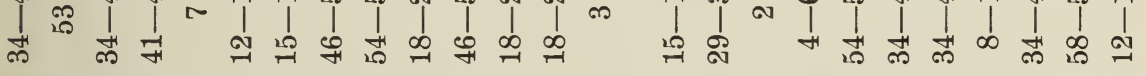

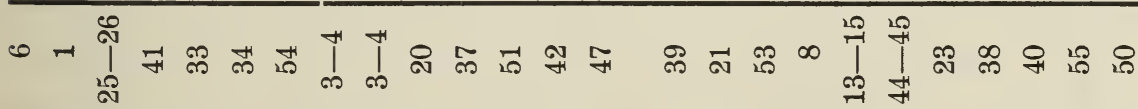

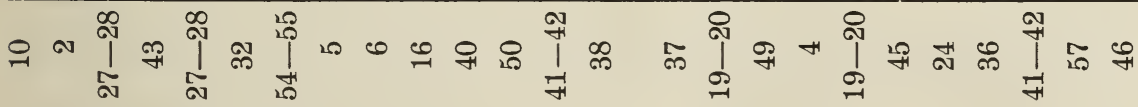

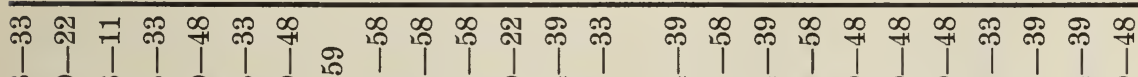

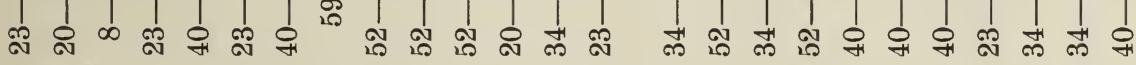

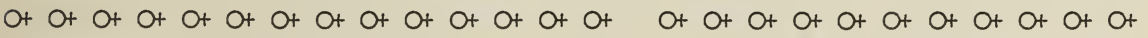

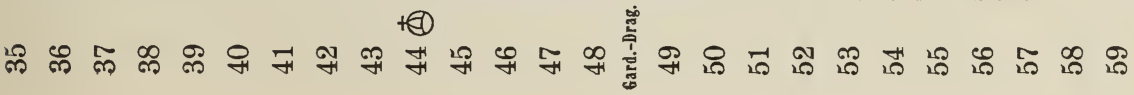




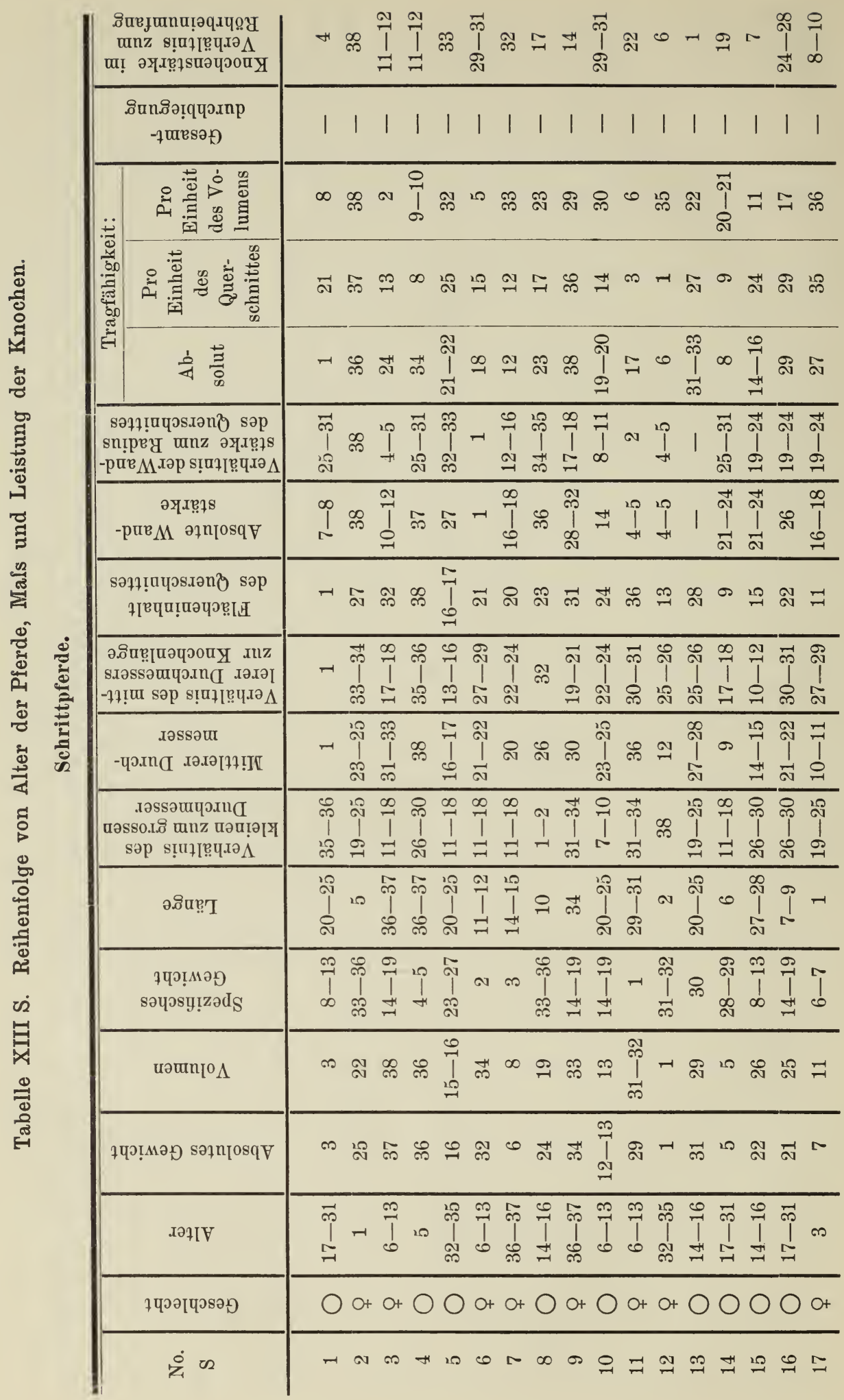




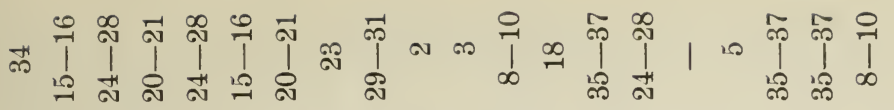

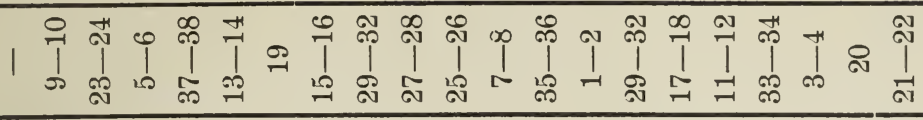

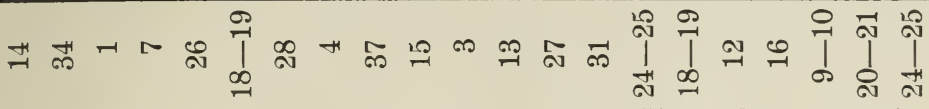

ㅇำ

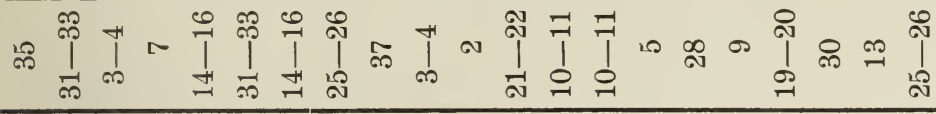

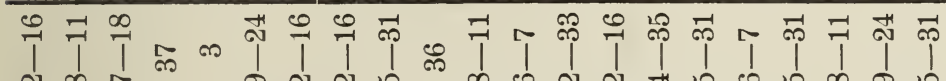

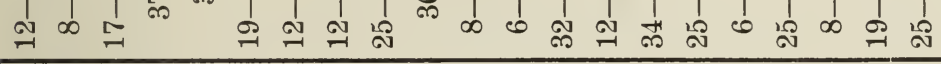

กำ

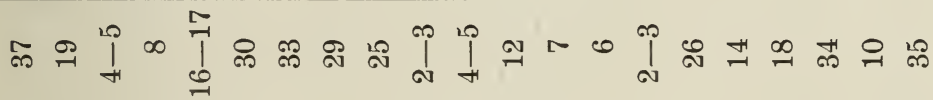

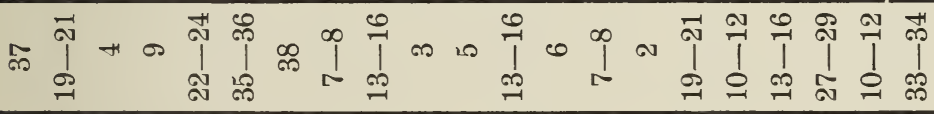

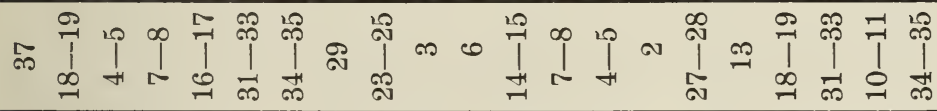

윽

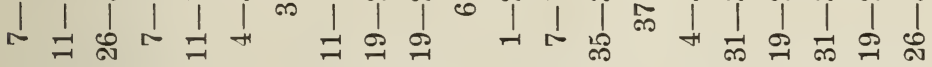

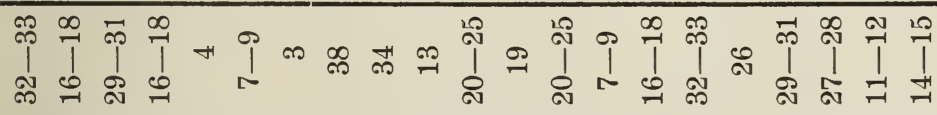

๓ สิ ฉ

更

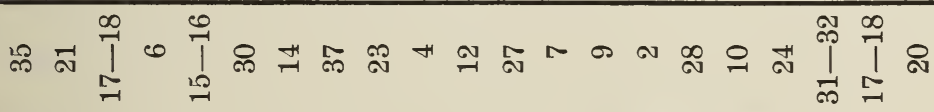

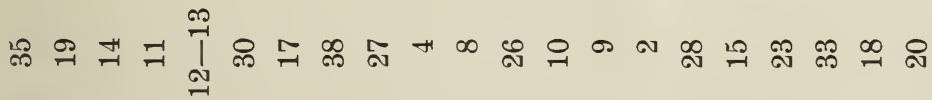

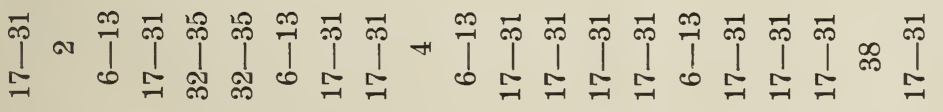

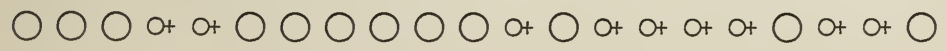

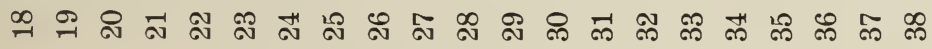


Tabelle XIV. Torsionsversuche.

\begin{tabular}{|c|c|c|c|c|c|c|c|c|c|c|}
\hline \multirow{2}{*}{$\begin{array}{l}\text { Bezeichnung des } \\
\text { Metacarpus: }\end{array}$} & \multicolumn{2}{|c|}{ A } & \multicolumn{2}{|c|}{ B } & \multicolumn{2}{|r|}{$\mathrm{C}$} & \multicolumn{2}{|c|}{$\mathrm{D}$} & \multicolumn{2}{|c|}{$\mathrm{E}$} \\
\hline & links & rechts & links & rechts & links & rechts & links & rechts & links & rechts \\
\hline Absolutes Gewicht $g$ & 540 & 545 & 603 & 596 & 694 & 702 & 673 & 680 & 563 & 573 \\
\hline Volumen $\mathrm{ccm}$. . . & 345 & 352 & 364 & 365,5 & 454 & 458 & 419 & 423 & 366 & 372 \\
\hline Spezifisches Gewicht & 1,57 & 1,55 & 1,66 & 1,63 & 1,53 & 1,53 & 1,61 & 1,61 & 1,54 & 1,54 \\
\hline Länge $\mathrm{mm}$. . . . . & 251 & 252 & 247 & 247 & 268 & 267 & 268 & 267 & 256 & 258 \\
\hline Kleiner \} Querschn.- $\mathrm{mm}$ & 32,4 & 33,2 & 29,2 & 29,4 & 32,8 & 32,8 & 31,7 & 32,1 & 31,9 & 32,5 \\
\hline Grosser $\int$ Durchmess. $\mathrm{mm}$ & 43,0 & 43,5 & 43,2 & 43,2 & 46,0 & ca. 46,0 & 44,9 & 44,0 & 45,3 & 45,7 \\
\hline $\begin{array}{l}\text { Die Belastung des } \\
50 \mathrm{~cm} \text { langen Radius: }\end{array}$ & & & rehung & betrug & $\begin{array}{l}\text { in de } \\
\text { in } G\end{array}$ & $\begin{array}{l}\text { en einzel } \\
\text { Graden: }\end{array}$ & nen $B$ & lastung & sstufen & \\
\hline $50-55 \mathrm{~kg} . .$. & 4 & 3 & 4 & 3 & 4 & 3 & 5 & 5 & 3 & 5 \\
\hline $55-60 " . . .$. & 5 & 4 & 4 & 4 & 6 & 5 & 6 & 5 & 6 & 4 \\
\hline $60-65 \% . .$. & 5 & 4 & 4 & 5 & 6 & 3 & 7 & 6 & 5 & 5 \\
\hline $65-70 " . .$. & 6 & 4 & 4 & 4 & 7 & 6 & 7 & 4 & 9 & 4 \\
\hline $70-75 n$ & 5 & 5 & 5 & 6 & 7 & 4 & 8 & 3 & 7 & 5 \\
\hline $75-80$ & 6 & 6 & 5 & 6 & 8 & 5 & 5 & 5 & 7 & 4 \\
\hline $80-85 n$ & - & 6 & 6 & 8 & 7 & 5 & 7 & 5 & - & 7 \\
\hline $85-90 "$ & - & - & - & 7 & 9 & 6 & 8 & 5 & - & - \\
\hline $90-95$ & - & - & - & - & 8 & 5 & 9 & - & - & - \\
\hline $95-100 "$. & - &. & - & - & 11 & 9 & 10 & - & - & - \\
\hline $100-105 \%$. & - & - & - & - & - & 8 & - & - & - & - \\
\hline $105-110 " .$. & - & - & - & - & - & 7 & - & - & - & - \\
\hline $110-115 " . \quad . \quad . \quad$. & - & - & - & - & - & 10 & - & - & - & - \\
\hline $\begin{array}{l}\text { Gesamttorsion in den } \\
\text { notierten Stufen in } \\
\text { Graden . . . . . }\end{array}$ & 31 & 32 & 32 & 43 & 73 & 76 & 72 & 33 & 37 & 34 \\
\hline $\begin{array}{l}\text { Der Bruch trat ein bei } \\
\text { einer Belastung des } \\
\text { Radius von } \mathrm{kg} . .\end{array}$ & 80 & 85 & 85 & 90 & 100 & 115 & 100 & 90 & 80 & 85 \\
\hline
\end{tabular}




\section{Lebenslaui.}

Ich wurde am 22. März 1866 zu Templin in der Mark geboren, besuchte die dortige Mittelschule und nach weiterer privater Vorbereitung das Seminar zu Oranienburg. Danach war ich bis Ostern 1901 als Lehrer tätig und bezog nun die Universität Jena, um Landwirtschaft zu studieren. Im Juli 1904 bestand ich dort das landwirtschaftliche Diplomexamen mit dem Prädikat „sehr gut" und wurde am 26. Juli 1906 auf Grund vorliegender Arbeit zur mündlichen Doktorprüfung zugelassen. 

